Journal of Patient-Centered

\title{
Abstracts From the 25th Annual Health Care Systems Research Network Conference, April 8-10, 2019, Portland, Oregon
}

Follow this and additional works at: https://aah.org/jpcrr

Part of the Analytical, Diagnostic and Therapeutic Techniques and Equipment Commons, Dietetics and Clinical Nutrition Commons, Diseases Commons, Health and Medical Administration Commons, Medical Specialties Commons, Mental and Social Health Commons, Public Health Commons, and the Translational Medical Research Commons

\section{Recommended Citation}

Abstracts from the 25th annual Health Care Systems Research Network Conference, April 8-10, 2019, Portland, Oregon. J Patient Cent Res Rev. 2019;6:52-126. doi: 10.17294/2330-0698.1706

Published quarterly by Midwest-based health system Advocate Aurora Health and indexed in PubMed Central, the Journal of Patient-Centered Research and Reviews (JPCRR) is an open access, peer-reviewed medical journal focused on disseminating scholarly works devoted to improving patient-centered care practices, health outcomes, and the patient experience. 


\section{Abstracts From the 25th Annual Health Care Systems Research Network Conference, April 8-10, 2019, Portland, Oregon}

The Health Care Systems Research Network (HCSRN) is made up of nonprofit health systems with embedded research departments whose scientists are dedicated to public domain research. The network's annual conference serves as a forum for research teams to disseminate study findings, stimulate new collaborations, and share insights about conducting research in realworld care settings. Abstracts accepted for presentation at HCSRN 2019 are published in this supplement of Journal of Patient-Centered Research and Reviews, the official scientific journal of the conference. (J Patient Cent Res Rev. 2019;6(1):52-126.)

\section{IMPLEMENTATION SCIENCE}

\section{OA1.01}

\section{Sustainment of Lean Redesigns for Primary Care Teams}

Dorothy Hung, ${ }^{1}$ Caroline Gray, ${ }^{2}$ Quan Truong, ${ }^{1}$ Michael Harrison ${ }^{3}$

${ }^{1}$ Palo Alto Medical Foundation Research Institute, Palo Alto, CA; ${ }^{2} V A$ Palo Alto Health Care System, Menlo Park, CA; ${ }^{3}$ Agency for Healthcare Research and Quality, Rockville, $M D$

Background: Quality improvements are notoriously followed by "backsliding" or relapse to the status quo. Although implementation of quality initiatives is well studied, there is less research on sustaining changes. This mixed-methods study examined the sustainment of Lean workflow redesigns for primary care teams several years after being introduced in a large, ambulatory care delivery system.

Methods: We conducted qualitative interviews of 57 organizational leaders and primary care providers, and fielded surveys to 1164 frontline physicians and staff in 17 primary care clinics across the system. We analyzed interviews and conducted independent sample t-tests to identify key factors facilitating the sustainment of new workflows among primary care teams. All analyses were conducted after Lean redesigns were implemented and scaled across the system in 3 consecutive phases - first in 1 pilot site, then in 3 "beta" sites, and finally in all remaining clinics.

Results: We found that the initial approach to implementing Lean redesigns in each clinic was critical to later sustainment. Adherence to Lean workflows was highest in the pilot clinic, despite having the longest postdesign measurement period. Pilot members reported having the highest levels of participation in designing workflows, were most highly engaged in quality improvement efforts, and held most favorable beliefs about Lean changes. Adherence to redesigns was lowest among those in the second beta phase of implementation; these members also reported highest levels of burnout. Overall facilitators of sustainment included active engagement of staff in designing new workflows, favorable beliefs about Lean changes and use of Lean management tools, and availability of time for improvement activities.

Conclusion: This study of Lean sustainment highlights the importance of early approaches to implementing change in primary care clinics. Our findings reinforce the important role of employee participation in design efforts, use of supportive management tools, and staff identification with organizational goals. Additional time and effort is required to fully involve staff in design efforts, but these time investments will likely help secure buy-in and adherence for the long term. Transformation of these core elements will facilitate the sustainment of Lean and similar innovations among primary care teams.

\section{OA1.02}

Implementation of a Direct-Mail FIT Program in Federally Qualified Health Centers: Screen to Prevent Colon Cancer (STOP CRC)

Edward Meich, ${ }^{1}$ Beverly B. Green, ${ }^{2}$ Jennifer L. Schneider, ${ }^{3}$ Sally Retecki, ${ }^{4}$ Tanya Kapka, ${ }^{4}$ Jennifer Coury, ${ }^{4}$ Erin Keast, ${ }^{3}$ Gloria Coronado, ${ }^{3}$ Amanda Petrik ${ }^{5}$

${ }^{1}$ Regenstrief Institute and Indiana University Center for Health Services and Outcomes Research, Indianapolis, IN; ${ }^{2}$ Kaiser Permanente Washington Health Research Institute, Seattle, WA; ${ }^{3}$ Kaiser Permanente Center for Health Research, Portland, OR; ${ }^{4}$ CareOregon, Portland, OR; ${ }^{5}$ Kaiser Permanente Northwest, Portland, OR 
Background: Evidence-based interventions can only be effective if successfully implemented; yet, little is known about the organizational factors that drive implementation success. Screen to Prevent Colon Cancer (STOP CRC), a randomized, pragmatic trial of a mailed fecal immunochemical test (FIT), is aimed at increasing colorectal cancer screening in 8 health centers in Oregon and California. The intervention improved rates of screening overall, yet substantial variation was observed in the extent that health centers implemented the intervention (ie, percentage of eligible adults who were mailed a FIT).

Methods: We evaluated drivers of implementation success in the inner and outer settings. We categorized the 8 health centers as having high, medium, and low implementation levels based on the proportion of eligible patients mailed a FIT. We applied configurational comparative methods (CCMs) to look across all program and clinic components and identify the implementation conditions with the strongest connections to implementation outcomes. Qualitative comparative analysis and coincidence analysis were used in $\mathrm{R}^{\circ}$ software. Using prior theoretical knowledge to assess this condition-level output, we identified a handful of factors that could distinguish between high, medium, and low implementation success.

Results: CCM analyses identified a 2-factor solution that, in combination, perfectly distinguished high vs medium vs low implementation with $100 \%$ consistency and $100 \%$ coverage. The 2 factors were centralized processing level (values: high; mixed) and separate intro letter (values: yes; no). Health centers with high levels of implementation had centralized staff and mailed the introductory letter separate from the FIT kit. Health centers with medium levels of implementation had 2 solution pathways for 1 ) the combination of centralized processing level $=$ mixed AND separate intro letter $=$ yes; or 2) the combination of centralized processing level = advanced AND separate intro letter $=$ no.

Conclusion: A centralized staffing model may have provided protected time for staff to implement the program. The mailing of an introductory letter separately from the FIT kit may have reflected a strong commitment to deliver all components of the program and maximize its effectiveness. Contrary to expectation, staffing changes, clinic growth, and training attendance did not predict implementation success. Applied use of CCM analyses can produce actionable findings to guide implementation and improve health care delivery.

\section{OA1.03}

Results From a Randomized Trial Comparing Strategies for Helping Community Health Centers Implement Guideline-Concordant Cardioprotective Care

Rachel Gold, ${ }^{1}$ Arwen Bunce, ${ }^{2}$ Stuart Cowburn, ${ }^{2}$ James Davis, ${ }^{1}$ Joan Nelson, ${ }^{2}$ Deborah Cohen, ${ }^{3}$ James Dearing, ${ }^{4}$ Michael Horberg ${ }^{1}$
${ }^{1}$ Kaiser Permanente Northwest, Portland, OR; ${ }^{2}$ OCHIN, Portland, OR; ' ${ }^{3}$ Oregon Health \& Science University, Portland, OR; ${ }^{4}$ Michigan State University, East Lansing, MI

Background: Statins can reduce cardiovascular disease (CVD) risk in patients with diabetes (DM), but statin prescribing often lags behind care recommendations. We compared how 3 increasingly intensive implementation strategies impacted community health centers' (CHCs) adoption of electronic health record (EHR) clinical decision support tools targeting guideline-concordant statin prescribing in DM. The EHR tools (the "CVD Bundle") were adapted from a previously successful intervention.

Methods: In this mixed-methods, pragmatic trial, $29 \mathrm{CHCs}$ from the OCHIN network were randomized to $3 \mathrm{arms}$, each of which received differing implementation support: 1) implementation toolkit (CVD Bundle use instructions; QI practice change techniques); 2) toolkit +2 -day in-person training with follow-up webinars; or 3) toolkit, training, webinars, plus practice facilitation. More than 300 additional OCHIN CHCs received no implementation support and were considered a control arm. The intervention occurred in July 2015. Using EHR data, we measured statin prescribing (per 2014 Joint National Committee and 2015 American Heart Association/American Diabetes Association guidelines) from 14 months preintervention through 34 months postintervention. We gathered qualitative data from the randomized $\mathrm{CHCs}$ via on-site observations and interviews, and regular phone calls.

Results: Statin prescribing trends increased from pre- to postintervention for all arms, but only arm 2 demonstrated a statistically significant change in trend compared to the control arm. Observed prescribing rates improved more in the study CHCs $(7.3 \%, 8.3 \%$, and $5.0 \%$ for arms 1,2 , and 3 , respectively) compared to the control arm (3.4\%). However, these differences did not follow an additive pattern. Qualitative data suggest these results can be explained by variation in staff EHR skills and leadership engagement; CHCs' practice-change culture; and EHR tool characteristics, which diminished their utility. In addition, the implementation strategies were not uniformly administered: the toolkit was infrequently used, webinar attendance was poor, staff turnover diminished training impacts, and few arm 3 clinics were prepared to "use" facilitation.

Conclusion: Commonly used implementation strategies resulted in minor improvement in CHCs' guidelineconcordant cardioprotective prescribing. Level of implementation support appeared less impactful than individual clinics' readiness to make targeted changes. Guideline dissemination efforts should start with formative evaluation of adopters' needs/preferences so that subsequently deployed implementation strategies are met with receptivity. 
OA1.04

Research Impact Optimization: Building on Existing
Research Impact Assessment Frameworks

Visanee Vicky Musigdilok, ${ }^{1}$ Brian Mittman, ${ }^{1}$ John Ovretveit $^{2}$

${ }^{1}$ Kaiser Permanente Southern California, Pasadena, CA; ${ }^{2}$ Karolinska Institutet, Stockholm, Sweden

Background: Interest in systematic assessment of research benefits and impacts has grown in response to tightening of national research budgets and increased calls for accountability in government spending. Multiple research impact frameworks have been introduced, ranging in breadth and specificity and the types of impacts included. We synthesized existing models to develop a comprehensive research impact pathway to 1) help researchers identify potential impacts in advance and guide actions to maximize realization of potential impacts, and 2) facilitate better understanding and achievement of research impacts for health system-based, health system-focused research conducted within a learning health care system.

Methods: We identified and synthesized existing research impact frameworks, reviewing the impact domains and metrics included and the underlying models depicting mechanisms or pathways of impact. The synthesis includes a fully elaborated research impact pathway relevant to health-related research and its impacts on key stakeholders and outcomes.

Results: The synthesized framework organizes research impact into 7 categories, incorporating the domains and structure most current models include to depict research output and uptake as well as a second dimension to address research implications for health care policy and practice. The framework covers quantifiable deliverables (eg, journal publications and grants) and depicts how research findings can lead to changes in multiple settings and levels to affect patient, provider, health system, and national-level policies, practices, and outcomes. Metrics are listed for all 7 categories, presented as broad descriptions. This approach allows researchers to identify and categorize expected outputs and to understand and facilitate their likely impact pathway.

Conclusion: Improved understanding of categories, mechanisms, and pathways of research impact will facilitate targeted efforts to maximize impacts and benefits, guiding researchers and other research stakeholders in knowing what types of actions are appropriate for different types of studies and anticipating and supporting their potential impacts. Many studies are "early stage" and will contribute to patient or system benefits only following significant additional research. Others can yield benefits in the near term but only if multiple actions are taken to ensure appropriate responses by policy and practice stakeholders. Explicit guidance to maximize impacts through these actions will strengthen societal return on research investment.
OA15.01

Novel Approaches to Measuring and Addressing the Common Challenges of Implementation and Long-Term Sustainability in SBIRT: Alcohol as a Vital Sign

Stacy Sterling, ${ }^{1}$ Thekla Ross, ${ }^{1}$ Constance Weisner, ${ }^{1}$ Wendy Lu, ${ }^{1}$ Sujaya Parthasarathy, ${ }^{1}$ Felicia Chi, ${ }^{1}$ Esti Iturralde ${ }^{1}$

${ }^{1}$ Division of Research, Kaiser Permanente Northern California, Oakland, CA

Background: Alcohol screening, brief intervention, and referral to treatment (SBIRT) is increasingly being implemented in health systems, but numerous challenges that can impact efficacy, effectiveness, and sustainability persist. We focus on challenges to SBIRT implementation and long-term sustainability and illustrate novel approaches being used to study them, in the context of a large-scale alcohol SBIRT initiative in Kaiser Permanente Northern California (KPNC), a large, integrated health care system.

Methods: The implementation of systematic alcohol SBIRT in adult primary care in KPNC was informed by an National Institute on Alcohol Abuse and Alcoholism-funded clusterrandomized implementation trial (ADVISe) of different modalities of SBIRT delivery. It involves electronic health record (EHR)-embedded, evidence-based alcohol screening instruments and clinical decision support tools; medical assistants and primary care providers (PCPs) trained in screening, brief intervention (BI) and referral techniques; and curricula and workflows developed for medical assistant and PCP training, skills reinforcement, and troubleshooting. Structures were created for measuring, communicating, and reinforcing provider- and facility-level EHR data on screening and BI performance.

Results: The initiative conducts an average of 145,000 alcohol screenings each month (on $86 \%$ of adult primary care patients) and 9500 BIs. Since its adoption in July 2013, there have been 8.7 million alcohol screenings conducted and 536,000 BIs delivered. However, there is significant variation in implementation outcomes (eg, BI rates varying from $32 \%$ to $83 \%$ across medical centers and from $0 \%$ to $100 \%$ among PCPs) across medical centers and clinics and among PCPs. Little is known about the factors causing this variability and those contributing to long-term sustainability of SBIRT. We describe a new approach to studying factors associated with successful SBIRT implementation and long-term SBIRT sustainability. Informed by the PRISM theoretical framework, this mixed-method study uses EHR data, PCP surveys, qualitative key informant interviews, and patient interviews to examine intervention-, organizational-, provider-, environmental-, and recipient-level factors, including assessments of patient and provider experiences of BI quality and fidelity. 
Conclusion: We discuss the challenges to successful implementation of universal SBIRT in health system settings and make recommendations for approaches to evaluating factors associated with its long-term sustainability.

\section{OA15.02}

\section{Costs of Program to Mail Fecal Immunochemical Tests to Medicare and Medicaid Members}

\author{
Richard Meenan, ${ }^{1}$ Laura-Mae Baldwin, ${ }^{2}$ Gloria \\ Coronado, ${ }^{1}$ Beverly B. Green,, ${ }^{3}$ Malaika Schwartz, ${ }^{2}$ \\ Jennifer Coury, ${ }^{4}$ Amanda Petrik ${ }^{1}$
}

${ }^{1}$ Kaiser Permanente Center for Health Research, Portland, OR; ${ }^{2}$ Department of Family Medicine, University of Washington, Seattle, WA; ${ }^{3}$ Kaiser Permanente Washington Health Research Institute, Seattle, WA; ${ }^{4}$ CareOregon, Portland, OR

Background: BeneFIT is a 4-year observational study of a mailed fecal immunochemical test (FIT) program implemented by two Medicaid/Medicare health plans in Oregon and Washington. BeneFIT compares two approaches to mailing FITs to enrollees: a collaborative model in which the health plan mails FITs and enrollees return FITs to their clinics for processing; and a centralized model of direct FIT mailings to enrollees who return the FITs to a centralized laboratory for processing. This paper compares Year 1 costs of developing and implementing the BeneFIT program across the two models and provides estimates of program costs per screened enrollee.

Methods: Organizational staff completed activity-based costing spreadsheets, assigning hours by intervention activity (eg, test processing) and job-specific wage rates. Nonlabor costs were from study data. Data matched each plan's development and implementation dates in 2015-2016 for Year 1; analyses were performed in 2017.

Results: Plan A (collaborative model) development costs were $\$ 11.5 \mathrm{~K}$, most of which were in administrative activities. Plan B (centralized model) development costs were $\$ 38.7 \mathrm{~K}$, with $42 \%$ allocated to FIT selection and mailing/tracking protocol design. Administrative tasks were similar across plans, although in Plan B, comparatively higher-paid director-level staff executed such tasks. Year 1 implementation costs were $\$ 55.2 \mathrm{~K}$ for Plan A and $\$ 113.2 \mathrm{~K}$ for Plan B, reflecting Plan B's greater enrollee outreach (8551 enrollees vs 2812). Labor costs were $55 \%$ of Plan A implementation costs, primarily in enrollee eligibility and processing of returned FITs. Plan B labor costs were 22\% of implementation costs, centered in mailing/tracking and test processing. Implementation cost per eligible enrollee was 57\% higher in Plan A (\$20.81) than in Plan B (\$13.25), reflecting Plan B's economies of scale. A similar proportion of completed FITs among screening-eligible patients in both plans led to a 51\% lower cost per completed FIT in Plan B
(\$72.75) vs Plan A (\$109.73). The 1054 additional completed FITs generated by Plan B (over Plan A) were produced at an average additional cost of $\$ 55$ (\$58,073/1054).

Conclusion: Results highlight the cost implications of adopting a collaborative vs centralized approach to implementing a health plan-based colorectal cancer screening intervention within two health plans serving Medicaid/Medicare populations.

OA15.03

Practice Facilitation to Improve Quality of Cardiovascular Disease Care in Small Independent Practices: A Comparative Case Study

Ann Nguyen, ${ }^{1}$ Allison Cuthel, ${ }^{1}$ Nancy Van Devanter, ${ }^{2}$ Thomas Gepts, ${ }^{1}$ Erin Rogers, ${ }^{1}$ Carolyn Berry, ${ }^{1}$ Donna Shelley ${ }^{1}$

${ }^{1}$ New York University School of Medicine, New York, NY; ${ }^{2} \mathrm{New}$ York University Rory Meyers College of Nursing, New York, NY

Background: Practice facilitation (PF) provides expertise to help primary care practices implement innovative care processes tailored to local context. HealthyHearts NYC (HHNYC), funded through Agency for Healthcare Research and Quality's EvidenceNOW initiative, tested the effectiveness of PF on the adoption of the Million Hearts ABCS cardiovascular disease evidence-based guidelines: appropriate aspirin use (A); blood pressure control (B); cholesterol management (C); and smoking cessation (S). Overall, the intervention implementation led to an increase in smokers counseled, but there were no statistically significant effects on A, B, and C. There was some variation, however, between practices. This study aimed to identify how context drove the PF process by exploring contextual differences between practices.

Methods: For this mixed-methods multiple-case study, data came from purposive sampling of 5 small independent practices representing 2 that consistently exceeded Million Hearts targets, 2 that improved from below to above, and 1 that never met targets. We used quantitative data from electronic health records, the Change Process Capability Questionnaire, and surveys of the practice, providers, and staff. We also used qualitative data from site observations and 15 interviews with the PF, provider, and staff. Interview guides and coding were informed by the Consolidated Framework for Implementation Research. Deductive (theory-driven) and inductive (open) approaches were used to code interviews.

Results: The practices had contextual differences that required PFs to tailor their processes. Six themes emerged that distinguished the practices. At the outer setting, practices varied in: 1) their reaction to external policies for payment reform; and 2) diverse immigrant and vulnerable patient populations. At the inner setting, they varied in: 3) 
communications and relationship between the lead provider and staff; 4) financial status and focus on incentives; 5) engagement of the provider with the PF; and 6) prioritization of competing quality improvement projects.

Conclusion: Despite their size, small independent primary care practices exhibit characteristics of a complex adaptive system. Context drives the PF process and thus requires the process to be substantially tailored to a practice's context. PFs should be prepared to align intervention implementations with practice priorities, be willing to provide out-of-scope information technology support, and invest time in building relationships and practice capacity.

\section{OA15.04}

\section{Adoption of Social Determinants of Health EHR Tools in Community Health Centers}

Rachel Gold, ${ }^{1,3}$ Erika Cottrell, ${ }^{2,3}$ Stuart Cowburn, ${ }^{3}$ Miguel Marino, ${ }^{2}$ Dagan Wright, ${ }^{3}$ Arwen Bunce, ${ }^{3}$ Ned Mossman, ${ }^{3}$ Marla Dearing, ${ }^{3}$ Mary Middendorf, ${ }^{3}$ Katie Dambrun, ${ }^{3}$ Inga Gruß, ${ }^{1}$ Nadia Yosuf, ${ }^{1}$ Molly Krancari ${ }^{3}$

${ }^{1}$ Kaiser Permanente Center for Health Research, Portland, OR; ' ${ }^{2}$ Oregon Health \& Science University, Portland, OR; ${ }^{3}$ OCHIN, Portland, OR

Background: Social determinants of health $(\mathrm{SDH})$ are nonclinical factors, such as housing, food security, and personal safety, that profoundly impact health risks and outcomes. Systematically documenting/acting on patients' SDH data in the electronic health record (EHR) could help eliminate disparities and promote health equity within community health centers' (CHCs) patients, who are especially susceptible to health impacts from SDH. However, adoption of EHR-based SDH documentation in $\mathrm{CHCs}$ has rarely been described.

Methods: We developed a suite of EHR tools for documenting and summarizing patient-reported SDH data and implemented these tools in OCHIN's national network of CHCs in June 2016. Descriptive analyses of the tools' adoption were conducted using extracted EHR data. Qualitative data were collected from $8 \mathrm{CHC}$ organizations with high tool-adoption rates, and content analysis was conducted.

Results: When the EHR's SDH tools went live in June 2016, 489 CHCs were on OCHIN's shared EHR; by study end (December 2017), it was 681 CHCs. During this period, 17,543 SDH screenings were conducted at 154 CHCs among 15,779 patients $(\cong 1 \%$ of patients seen during that period). Screenings most commonly occurred in office visits with established patients ( $42 \%$ of screenings), followed by pediatric preventive medicine visits (14\%). Respondents answered all $7 \mathrm{SDH}$ domains (listed below) in $30 \%$ of screening occurrences. Several CHCs only collected information on a single domain. Among patients who responded to specific questions, $52 \%$ reported financial strain, 35\% food insecurity, 19\% housing insecurity, 14\% risk for interpersonal violence, $67 \%$ insufficient physical activity, $67 \%$ social isolation, and $59 \%$ stress. Numerous barriers to SDH documentation were identified, including how to identify which patients with SDH needs want help addressing those needs, how to connect patients with SDH needs to local resources, and complex workflow issues.

Conclusion: To ensure widespread EHR documentation of $\mathrm{SDH}$ needs in primary care $\mathrm{CHCs}$, as is widely recommended, implementation barriers to such documentation must be identified and addressed. The results presented here can inform future efforts to build our understanding of implementation strategies for SDH data collection in CHCs.

\section{P10.01}

Referral to and Use of a Pharmacy-Led Opioid Tapering Program: Interviews With Clinicians and Pharmacists

Alison Firemark, Jennifer L. Schneider, ${ }^{1}$ Dea Papajorgji-Taylor, ${ }^{1}$ Jennifer Kuntz, ${ }^{1}$ Xiuhai Yang, ${ }^{1}$ John Dickerson, ${ }^{1}$ Lou Ann Thorsness, ${ }^{2}$ Katherine Reese, ${ }^{2}$ Mark Sullivan, ${ }^{3}$ Lynn Debar, ${ }^{4}$ Dave Smith ${ }^{1}$

${ }^{1}$ Kaiser Permanente Center for Health Research, Portland, OR; ${ }^{2}$ Clinical Pharmacy Services, Kaiser Permanente Northwest, Portland, OR; ${ }^{3}$ University of Washington, Seattle, WA; ${ }^{4}$ Kaiser Permanente Washington Health Research Institute, Seattle, WA

Background: Responding to growing concerns regarding chronic high-dose opioid use, Kaiser Permanente Northwest (KPNW) implemented a pharmacist-led program to support patients tapering off opioid medications - the Support Team Onsite Resource for Management of Pain (STORM) program. Through interviews with the STORM team and primary care providers (PCPs), we identified barriers and facilitators for referring patients to this resource.

Methods: We developed interview guides and conducted interviews by phone or in-person. Interviews were audiorecorded and transcribed. Content analysis of transcripts resulted in identifying key themes on reasons why PCPs refer or do not refer to the STORM program.

Results: We completed 35 qualitative interviews: 15 pharmacists and program staff, and 20 PCPs. Pharmacists and PCPs both acknowledged that complex patients on high doses of opioids who may require more attention than the PCP is able to provide are likely to be referred to the program. PCPs also identified unique reasons for referrals, such as patient fear/reluctance to reduce opioid dosage, violation of an opioid treatment plan, and patient request to taper. Pharmacists observed that PCPs are likely to refer when PCP has a large number of patients in need of taper, has previous positive experience with the STORM program, or is a newer provider to the health plan. Pharmacists and PCPs both identified common barriers for referral and use of the STORM program: PCP or patient preference for PCP to 
manage the taper; forgetfulness of the resource; and confusing STORM with other pain management resources. Pharmacists specifically observe that PCPs less likely to refer are comfortable managing the taper on their own or are resistant to being told their patients need to be tapered, while PCPs explain that lack of direct access to a STORM pharmacist in their clinic for consultation may reduce referral practices.

Conclusion: Interview findings elucidate under what circumstances PCPs are likely to refer patients to the STORM program for assistance in lowering or coming off from opioid medications and identifies several areas that could improve referrals to the service, including increased program presence in clinics and reminders to PCPs about the nature and scope of the STORM program.

\section{P10.02}

\section{Implementing an Evidence-Based Guideline in Dental Offices}

Inga Gruß, ${ }^{1}$ Jeff Fellows, ${ }^{1}$ Charles Kaplan, ${ }^{2}$ Erick Guerrero, ${ }^{2}$ Deborah Polk ${ }^{3}$

${ }^{1}$ Kaiser Permanente Center for Health Research, Portland, OR; ${ }^{2}$ University of Southern California, Los Angeles, CA; ${ }^{3}$ University of Pittsburgh, Pittsburgh, PA

Background: Fostering the implementation of evidencebased guidelines in large, multisite dental practices can improve oral health of patients. The pit-and-fissure guideline, issued by the American Dental Association (ADA), recommends the use of dental sealants to prevent and arrest incipient caries on occlusal tooth surfaces. Sealant placement rates, and thus guideline adherence, remain low among dental providers. This qualitative study assessed existing implementation strategies and barriers and facilitators for ADA's pit-and-fissure guideline in Kaiser Permanente Northwest (KPNW) dental offices.

Methods: We followed a grounded-theory approach, continuously analyzing qualitative data to inform the next steps in the data collection process. We conducted semistructured interviews with 5 members of the leadership team, observed clinical routines in 4 dental offices, and engaged 30 dental professionals in focus groups in 3 dental clinics. Data collection aimed at learning about existing implementation strategies for clinical guidelines, with a focus on the pitand-fissure guideline. We also assessed organizational and practitioner barriers and facilitators to implementing the pitand-fissure guideline in dental offices.

Results: In KPNW, guideline selection and implementation are managed by a clinical effectiveness committee (CEC) consisting of one dentist from each dental office. The CEC is tasked with screening, selecting, and recommending guidelines for implementation into clinical care. Guidelines might be integrated into a set of annual performance and quality targets, with each dental office managing the implementation of selected guidelines into clinical care based on local factors. Barriers to implementing the pit-andfissure guideline included limited knowledge of the guideline content, particularly for treatment of incipient caries; lack of ownership of the process; and limited resources to accomplish additional care steps. Facilitators included a recognition of the importance of following evidence-based guidelines, well-functioning workflows within existing subspecialties, and openness to receiving prompts and support to accomplish the evidence-based care goals.

Conclusion: Qualitative analysis can identify important organizational and clinical barriers and facilitators affecting the implementation of evidence-based practice. Processes to screen, select, and recommend guidelines are necessary but not sufficient to successful implementation. A robust implementation structure is necessary to facilitate the implementation of guidelines into routine clinical practice.

\section{P10.03 \\ Implementation of Care Management Intervention for Patients With Chronic Obstructive Pulmonary Disease at Risk of Acute Exacerbation Across a Large Integrated Health Care Organization}

Richard Mularski, ${ }^{1,3}$ David Mosen, ${ }^{1}$ Sanja Uskokovic, ${ }^{2}$ Bryan Skalberg, ${ }^{2}$ Achikam Haim, ${ }^{2}$ Marianne Turley, ${ }^{2}$ Nancy Lee, ${ }^{2}$ Wui-Leong Koh ${ }^{3}$

${ }^{1}$ Kaiser Permanente Center for Health Research, Portland, OR; ${ }^{2}$ Kaiser Permanente Northwest, Portland, OR; ${ }^{3}$ Northwest Permanente, P.C., Portland, OR

Background: Chronic obstructive pulmonary disease (COPD) affects 12 million to 29 million individuals in the United States and is responsible for 800,000 yearly hospitalizations. Deficits in implementation of treatments for acute exacerbations (AEs) are well documented. We developed and tested an implementation project that used a targeted intervention across an integrated health care organization to identify at-high-risk individuals, provide guideline-recommended proactive care, and evaluate process and outcome metrics of the care delivery for this population.

Methods: We identified an at-risk COPD population in two phases for implementation. Phases were defined as 1) age > 65 years by ICD- 10 visits and 2 or more AEs in the prior year with systemic steroid dispensing (phase I); or 2) expanding to age $>40$ years with any COPD AE hospitalizations in the prior year (phase II). Care management teams competed an action plan with patients (standardized symptoms linked to actions, like start medications) and facilitated flu/ pneumococcus vaccine completion and orders for rescue medications. Process measures assessed successful delivery of care components; outcome metrics included AEs, utilization, and death, comparing to historical controls and as completers (received all planned interventions/meds) vs non-completers. Results: We identified 149 patients in phase I and 264 in phase II; respective historical cohorts had 118 and 149 
patients. Action plans were delivered in $55 \%$ of outreached patients, vaccine rates improved from $<50 \%$ to $65 \%$ $(\mathrm{P}<0.01)$, and rescue medication orders improved from $<1 \%$ to $20 \%(\mathrm{P}<0.0001)$. Nonsignificant trends were seen in hospital admissions (26\% postimplementation vs $31 \%$ prior) and 30-day readmission (18\% vs 35\%). Total inpatient hospital days were reduced in the combined analyses of both phases of intervention in those completers $(0.94 \pm 2.51$ days) who received all components compared to those in the implementation group who did not receive all components $(1.90 \pm 5.58$ days; $\mathrm{P}<0.05)$. Similar trends were seen in utilization. In the completer group, mortality trended down from $6.0 \%$ to $1.3 \%(\mathrm{P}=\mathrm{ns})$ and overall AEs from $25.0 \%$ to $20.5 \%(\mathrm{P}=\mathrm{ns})$ compared to non-completers.

Conclusion: Care management intervention improved care delivery for COPD patients. Total inpatient hospital days were lower for intervention completers $(\mathrm{P}<0.05)$, with favorable trends in utilization, AEs, and death (not statistically significant).

\section{P10.04}

\section{Can Guidelines Support Deimplementation of Computed Tomography for a Common Condition? Trends in Diagnostic Evaluation for Hematuria Within and Across Health Care Systems}

\section{Matthew Nielsen, ${ }^{1,4}$ Michael Parchman, ${ }^{2}$ Marilyn Kwan, ${ }^{3}$ Jenny Staab, ${ }^{4}$ Sheila Weinmann ${ }^{4}$ \\ ${ }^{1}$ The University of North Carolina at Chapel Hill, Chapel Hill, NC; ${ }^{2}$ Kaiser Permanente Washington Health Research Institute, Seattle, WA; ${ }^{3}$ Kaiser Permanente Northern California, Oakland, CA; ${ }^{4}$ Kaiser Permanente Northwest, Portland, OR}

Background: Utilization of computed tomography (CT) in the United States has soared, from 3 million scans per year in 1985 to more than 85 million in 2010, leading to concerns about overutilization. Hematuria is prevalent, with more than 2 million U.S. patients referred to urologists annually for evaluation. Historical U.S. guidelines recommend CT for all adults with any degree of hematuria, whereas international guidelines recommend ultrasound, explicitly citing concerns about cost-effectiveness and radiation harms. Against this backdrop, physicians in Kaiser Permanente (KP) developed a new risk-stratified guideline for hematuria, with CT restricted to patients at highest risk [gross hematuria $(\mathrm{GH})$, renal ultrasound for patients with microhematuria and risk factors [(MHRF) + tobacco exposure, male sex, women over 50 years old)], and no evaluation for nonsmoking women less than 50 years old with low-risk microhematuria (LRMH). Methods: 156,926 patients with hematuria in 3 health care systems from 2010 to 2016 were identified. Two systems (KP Northern California [KPNC] and KP Northwest [KPNW]) adopted the new risk-stratified guideline in 2012-2013; the third system, KP Washington (KPWA), did not adopt any specific guideline during the study period. We compared trends in CT utilization within and between systems among the 3 risk groups before (2010-2011) and after (2014-2015) guideline adoption.

Results: Among patients receiving evaluation $(n=81,291)$, the system with highest baseline CT utilization (KPNC) had stable utilization of $\mathrm{CT}$ for $\mathrm{GH}$ patients $(74.7 \%$ to $73.8 \%$, $\mathrm{P}=0.2301)$ and substantial decreases in CT utilization for MHRF patients $(67.6 \%$ to $44.5 \%, \mathrm{P}<0.0001)$ and LRMH patients $(52.1 \%$ to $43.8 \%, \mathrm{P}<0.0001)$ after adoption of the new guideline. The system with lowest baseline CT utilization (KPNW) had guideline-concordant increase in CT utilization for $\mathrm{GH}$ patients $(22.0 \%$ to $68.3 \%, \mathrm{P}<0.0001)$ but also guideline-discordant increase in $\mathrm{CT}$ for MHRF patients (21.7\% to $36.7 \%, \mathrm{P}<0.001)$ and LRMH patients $(21.0 \%$ to $32.1 \%, \mathrm{P}=0.0022)$. KPWA had more modest changes in $\mathrm{CT}$ utilization (GH: $55.8 \%$ to $58.9 \%, \mathrm{P}=0.0567$; MHRF: $43.0 \%$ to $48.7 \%, \mathrm{P}<0.0001$; LRMH: $42.5 \%$ to $42.0 \%, \mathrm{P}=0.8902$ ).

Conclusion: Adoption of a risk-stratified guideline was associated with differential changes in utilization within and across health systems, sensitive to baseline CT utilization and patient risk factors. Substantial residual variation and guideline-discordant utilization suggest additional opportunities for improvement.

\section{P10.05}

System-Level Challenges and Successes of a Pharmacy-Led Opioid Tapering Program: Interviews With Clinicians and Pharmacists

Jennifer L. Schneider, ${ }^{1}$ Alison Firemark, ${ }^{1}$ Dea Papajorgji-Taylor, ${ }^{1}$ Jennifer Kuntz, ${ }^{1}$ John Dickerson, ${ }^{1}$ Xiuhai Yang, ${ }^{1}$ Mark Sullivan, ${ }^{2}$ Lou Ann Thorsness, ${ }^{3}$ Lynn Debar, ${ }^{4}$ Katherine Reese, ${ }^{3}$ Dave Smith ${ }^{1}$

${ }^{1}$ Kaiser Permanente Center for Health Research, Portland, OR; ${ }^{2}$ University of Washington, Seattle, WA; ${ }^{3}$ Clinical Pharmacy Services, Kaiser Permanente Northwest, Portland, OR; ${ }^{4}$ Kaiser Permanente Washington Health Research Institute, Seattle, WA

Background: Responding to growing concerns regarding chronic high-dose opioid use, Kaiser Permanente Northwest implemented a pharmacist-led, phone-based program to support patients tapering off opioid medications. Our study examines the reach, adoption, and implementation of the Support Team Onsite Resource for Management of Pain (STORM) program. To understand implementation factors and associated barriers of the program, we conducted indepth interviews with staff delivering the program and referring primary care providers (PCPs).

Methods: Participants were recruited via email, and interviews were conducted in-person or by phone using an interview guide informed by the RE-AIM framework. Interviews were recorded, transcribed, and content-analyzed 
by trained qualitative staff, resulting in refined themes.

Results: Thirty-five interviews were completed - 15 pharmacists/program staff, and 20 PCPs. Challenges to program implementation identified by both PCPs and pharmacy staff included occasional delays in initial outreach due to workload or lack of staffing, and limitations in offering nonpharmacological pain management resources to patients. Pharmacy staff specifically described experiencing occasional burnout and observed unclear PCP-patient communication about the purpose of the referral. Some PCPs cited lack of understanding regarding how the referral process works and how STORM services differ from other pain management resources in the system. PCPs and pharmacists identified multiple common strengths of STORM: the training and empathetic skill set of pharmacists; thorough documentation of the taper process within the health record; reducing PCP workload burden; and an individualized taper approach allowing pharmacists to build trust and rapport with patients. Pharmacists further identified the structure of the STORM program as an important success factor, including referral triage and their collaborative team-based approach. PCPs find beneficial the ability to have on-site consultations with pharmacists and view the program as an essential service for managing patients with complex care needs.

Conclusion: Interviewees describe STORM as an invaluable resource for both PCPs and patients by delivering compassionate patient-centered care in lowering opioid doses. More program implementation strengths than challenges were noted by interviewees. Identifying system-level factors important to the success and ongoing improvement of the STORM program may be useful for other health systems considering a similar opioid tapering program.

\section{P10.06}

Positive Deviance Approaches to Improving Vaccination Coverage Rates Within Health Care Systems: A Systematic Review

\author{
Sonal Singh, ${ }^{1}$ Kathleen M. Mazor, ${ }^{1}$ Kimberly A. Fisher ${ }^{1}$ \\ ${ }^{1}$ University of Massachusetts Medical School / Meyers \\ Primary Care Institute, Worcester, MA
}

Background: Vaccination is effective in decreasing preventable morbidity and mortality. While RCTs have demonstrated effective interventions under controlled conditions, vaccination rates continue to fall short of targets. Practical strategies that can be implemented successfully in real-world settings are needed. The positive deviance approach is based on the principle that studying individuals or organizations that consistently demonstrate a high level of performance can reveal such strategies. We conducted a systematic review to identify strategies to improve vaccination coverage using the positive deviance approach. Methods: In October 2017, we searched English-language articles in Medline, Embase, Cochrane Library, CINAHL, and PsycINFO without any date restrictions. Two reviewers independently and in duplicate reviewed each citation for potential eligibility. We included positive deviance studies of any design (qualitative, quantitative, and mixed methods). We extracted data on the strategies and elements identified as critical to success and conducted qualitative synthesis of the findings. We also evaluated the quality of studies using the 14-item QATSDD tool. Our systematic review was registered at PROSPERO (2017 CRD42017057040).

Results: We reviewed 241 citations and included 8 studies conducted in diverse locations, including United States, United Kingdom, and Africa. The studies evaluated several vaccines including H1NI, diphtheria-pertussis-tetanus, human papillomavirus, influenza, polio, measles-mumpsrubella, Haemophilus influenzae type b, and hepatitis B. The settings included schools, practice sites, local health departments, and Veterans Affairs medical centers as well as countrywide evaluations of immunization programs in Africa. The reporting of quality elements was variable. High-performing organizations described the key components of the vaccination process as including a) planning and prioritization, b) efforts to increase awareness, c) increasing access and convenience of vaccination, and d) program evaluation including data review. The structure (eg, equipment) and context (eg, community engagement, leadership involvement, team work, organizational climate) supported the process of vaccination. Limitations include the lack of hypothesis testing in most studies and identification of positive deviants via peers rather than objective measures. Conclusion: By synthesizing the findings of studies that apply the positive deviance approach to vaccination, we uncovered practical, real-world, efficient strategies to improve vaccination across diverse settings. Efforts to improve vaccination rates could consider these strategies to reduce the burden of vaccine preventable diseases.

\section{MENTAL HEALTH, ALCOHOL AND SUBSTANCE ABUSE/USE}

\section{OA2.01 \\ Depression Screening and Treatment Among Disparity Populations in Primary Care: A Retrospective Analysis of Electronic Health Record Data}

\author{
Katherine Sanchez, ${ }^{1}$ Kiumars Zolfaghari, ${ }^{1}$ Afsaneh \\ Rezaeizadeh ${ }^{1}$ \\ ${ }^{1}$ Baylor Scott \& White Health, Dallas, TX
}

Background: Depression is the leading cause of medical disability, health burden, and increased medical cost in the United States and the world. Ethnic minorities have significantly lower odds of receiving adequate 
antidepressant treatment, guideline-concordant care, and experience a disproportionate burden of disability. Despite recommendations from the U.S. Preventive Services Task Force that primary care providers screen all adult patients for depression, overall rates of depression screening remain low, with racial minorities half as likely to be screened compared with whites.

Methods: We conducted a retrospective observational study of about 37,000 patient visits across 8 Baylor Scott \& White clinics in the Dallas/Fort Worth metroplex in 2017. Patient demographics and clinical information, including PHQ-2 and PHQ-9 scores, depression diagnosis, and behavioral health visits, were collected from the electronic health record. Descriptive analysis included means and frequencies. Multivariate analyses are in progress.

Results: The study cohort comprised 11,803 patients, majority female (64.3\%), with an average age of 48.0 years (standard deviation: 12.8). Of these, 63\% were Hispanic, the majority did not speak English, and 21\% were black. The depression screening rate at the clinics was $62 \%$ of all patients at annual visit. Of those patients, 2228 (28\%) had a score greater than 4 on the PHQ-9 (indicating the presence of depression) and only half of those had a visit with the licensed clinical social worker. Importantly, among patients screened for depression (PHQ-2), 30\% were diagnosed but $22 \%$ of those did not have a PHQ-9 on record. Additionally, out of 2228 patients with full PHQ-9 greater than 4 in 2017, only $450(20.2 \%)$ patients had a follow-up PHQ-9 within 6 months of their first PHQ-9.

Conclusion: Since appropriate management of depression calls for all patients diagnosed with depression, and particularly those treated with antidepressant medications, to have an assessment of symptoms using a validated depression symptom measure, findings represent a deviation from guideline-concordant care. The practice transformation necessary for broad implementation of evidence-based depression screening and treatment guidelines into practice will require analysis of the contextual factors to overcome barriers, especially those related to organizational readiness and mechanisms to support implementation and sustain integrated health care programs.

\section{OA2.02 \\ Development and Validation of Clinical Predictive Models for the Emergence of Alcohol or Other Drug Use Problems Between 12 and 18}

\author{
Felicia Chi, ${ }^{1}$ Stacey Alexeeff, ${ }^{1}$ Joseph A. Boscarino, ${ }^{2}$ \\ Ryan Dugan, ${ }^{2}$ Beth Waitzfelder, ${ }^{3}$ Timothy Frankland, ${ }^{3}$ \\ Brian Ahmedani, ${ }^{4}$ Yong Hu, ${ }^{4}$ Amy Loree ${ }^{4}$ \\ ${ }^{1}$ Kaiser Permanente Northern California, Oakland, CA; \\ ${ }^{2}$ Geisinger Health, Danville, PA; ${ }^{3}$ Kaiser Permanente Hawaii, \\ Honolulu, HI; ${ }^{4}$ Henry Ford Health System, Detroit, MI
}

Background: Alcohol or other drug (AOD) problems and their associated consequences have significant impacts for many adolescents and their families. Early identification and intervention can be beneficial, yet practical tools for facilitating identification in clinical settings is lacking. This study aims to develop and validate a predictive model of adolescent AOD problem emergence, using clinical and demographic data from a birth cohort identified across 4 health systems.

Methods: This observational, electronic health records (EHR)-based retrospective cohort study identified a birth cohort of 41,176 adolescents born between 1997 to 2000 in 4 health systems (Kaiser Permanente Hawaii, Kaiser Permanente Northern California, Geisinger Health, and Henry Ford Health System), with continuous membership since birth, allowing for a 12-month coverage gap. For the adolescents and their mothers, data on demographics, socioeconomic status, diagnoses, risk behaviors, prescriptions, and service utilization from birth through the adolescent's 12 th birthdate were extracted from the health plans' EHRs. The outcome was development of an AOD use disorder between ages 12 and18, defined as either 1) a contact with the AOD treatment program, or 2) receiving a nontobacco AOD diagnosis. We conducted Cox proportional hazard models using Kaiser Permanente Northern California data to develop a predictive model for AOD problem development by age 18 and validated the model using combined data from the other 3 sites.

Results: In addition to birth year, adolescents' gender, race/ethnicity, and ever-Medicaid-insured status as well as diagnoses of attention-deficit/hyperactivity disorder (ADHD), bed-wetting, bipolar disorders, conduct disorders, depression, headache, major depression, migraine, oppositional defiant disorder, and trauma- or stress-related disorders by age 12 were significantly associated with AOD problem development by age 18 . The presence of maternal alcohol or any drug use disorders, chronic pain, intimate partner violence, major depression, minor depression, obsessive-compulsive disorders, or tobacco use disorders also conferred significantly higher risk for adolescents' AOD problem development. Stratified analyses identified gender-specific risk factors.

Conclusion: Predictive models of this kind may be useful for targeted prevention and early intervention efforts by clinicians and health policymakers and clinical research.

OA2.03

Antidepressant Continuation in Pregnancy in Relation to Infant Birthweight

Paige D. Wartko, ${ }^{1}$ Noel S. Weiss, ${ }^{2}$ Daniel A. Enquobahrie, ${ }^{2}$ K.C. Gary Chan, ${ }^{3}$ Alyssa StephensonFamy, ${ }^{4}$ Beth A. Mueller, ${ }^{2}$ Sascha Dublin ${ }^{1}$ 
${ }^{1}$ Kaiser Permanente Washington Health Research Institute, Seattle, WA; ${ }^{2}$ Department of Epidemiology, University of Washington, Seattle, WA; ${ }^{3}$ Department of Biostatistics, University of Washington, Seattle, WA; ${ }^{4}$ Department of Obstetrics and Gynecology, University of Washington, Seattle, WA

Background: Every year, $6 \%-7 \%$ of pregnant U.S. women $(\sim 300,000$ women) use antidepressants. Many studies have assessed antidepressant use during pregnancy in relation to risk of smaller infant birthweight; however, most did not account for potential confounding by mental health status or assess risk separately for male and female infants.

Methods: We addressed this question among women using antidepressants before pregnancy in a retrospective cohort study using electronic health data and linked Washington State birth records of singleton, live births from 2001 to 2014 to women enrolled in Kaiser Permanente Washington. The study included women with 1 or more antidepressant prescription filled up to 6 months before pregnancy. Women with 1 or more antidepressant fill during pregnancy were considered "exposed" $(\mathrm{n}=1772)$; those without were "unexposed" ( $\mathrm{n}=1249)$. We calculated mean differences (MD) in infant birthweight and relative risks (RR) of small or large size for gestational age $(<10$ th or $>90$ th percentile, respectively, for gestational age- and sex-specific growth curves), low birthweight ( $<2500$ grams), and macrosomia ( $>4500$ grams) using inverse probability of treatment weighting to account for baseline characteristics, including mental health status.

Results: Female infants of women who continued antidepressant use in pregnancy had lower mean birthweight compared with those who did not continue (MD: -105.4 g, 95\% CI: -163.0 to $-47.7 \mathrm{~g}$ ), whereas male infants had a smaller, nonsignificant difference (MD: $-50.8 \mathrm{~g}, 95 \%$ CI: -108.6 to $7.1 \mathrm{~g}$ ). We observed increased risk of being small for gestational age among female infants of women continuing antidepressants (RR: $1.65,95 \% \mathrm{CI}: 1.09$ to 2.50 ) but not among male infants (RR: $0.86,95 \%$ CI: 0.58 to 1.27). After restricting continuers to women with fills in all trimesters, among female and male infants combined, we observed decreased risk of macrosomia (RR: $0.49,95 \% \mathrm{CI}$ : 0.25 to 0.99 ) and being large for gestational age (RR: 0.68 , 95\% CI: 0.52 to 0.91 ) among continuers compared with discontinuers.

Conclusion: After accounting for maternal characteristics, including mental health status, we observed greater risk of small for gestational age specific to female infants; however, these findings must be replicated and considered in conjunction with other risks and benefits associated with antidepressant use in pregnancy, including the woman's mental health.
OA2.04

Trends in Marijuana Use Among Pregnant Women With and Without Nausea and Vomiting in Pregnancy, 2009 to 2016

Kelly C. Young-Wolff, ${ }^{1}$ Varada Sarovar, ${ }^{1}$ Lue-Yen Tucker, ${ }^{1}$ Lyndsay A. Avalos, ${ }^{1}$ Stacey Alexeeff, ${ }^{1}$ Amy Conway, ${ }^{2}$ Mary Anne Armstrong, ${ }^{1}$ Constance Weisner, ${ }^{1}$ Cynthia I. Campbell, ${ }^{1}$ Nancy Goler ${ }^{3}$

${ }^{1}$ Division of Research, Kaiser Permanente Northern California, Oakland, CA; ${ }^{2}$ Early Start Program, Kaiser Permanente Northern California, Oakland, CA; ${ }^{3}$ Regional Offices, Kaiser Permanente Northern California, Oakland, $C A$

Background: Even though previous cross-sectional studies indicate higher marijuana use among women with nausea and vomiting during pregnancy (NVP), it is unknown whether the trends in marijuana use over time differ by NVP status because prenatal use is increasing in general as the drug becomes more socially acceptable.

Methods: Using data from Kaiser Permanente Northern California (KPNC), we analyzed the trend in marijuana use in pregnant women, $>12$ years of age, who completed a selfadministered questionnaire on marijuana use and a urine toxicology test for cannabis during standard prenatal care, from 2009 to 2016. Utilizing a Poisson regression with a log link function, and adjusting for sociodemographics and parity, we estimated the annual prevalence of marijuana use for those with and without NVP. We tested for linear trends and differences in trends by NVP.

Results: Of 220,510 pregnancies, 38,831 (17.6\%) had an NVP diagnosis. Prenatal marijuana use was elevated each year among women with NVP. The adjusted prevalence of use increased significantly from 2009 to 2016 at an annual rate of 1.086 (95\% CI: 1.069-1.104) among women with NVP, from $6.5 \%$ (95\% CI: $5.7 \%-7.2 \%)$ to $11.1 \%$ (95\% CI: 10.2\%-12.0\%), and 1.069 (95\% CI: 1.059-1.080) among women without NVP, from $3.4 \%$ (95\% CI: $3.2 \%-3.7 \%$ ) to $5.8 \%$ (95\% CI: $5.5 \%-6.1 \%)$. Trends did not vary by NVP status.

Conclusion: From 2009 to 2016, prenatal marijuana use among KPNC pregnant women increased irrespective of their NVP status; however, the prevalence was consistently higher among those with NVP. Since the effects of marijuana on pregnancy outcomes are still uncertain, clinicians should advice pregnant women to use alternative, evidence-based interventions to alleviate NVP and ask them to abstain from using prenatal marijuana. 
OA9.01

"What Will Happen If I Say Yes?" Perspectives Among Adults With Depressive Symptoms on Routine Questions About Firearms Access in Primary Care

Julie Richards, ${ }^{1}$ Sarah Hohl, ${ }^{2}$ Courtney Segal, ${ }^{2}$ Casey Luce, ${ }^{1}$ Ursula Whiteside, ${ }^{3}$ Emily Williams, ${ }^{4}$ Greg Simon, ${ }^{1}$ Amy Lee, ${ }^{1}$ Rob Penfold, ${ }^{1}$ Evette Ludman, ${ }^{1}$ David Grossman ${ }^{1}$

${ }^{1}$ Kaiser Permanente Washington, Seattle, WA; ${ }^{2}$ University of Washington, Seattle, WA; ${ }^{3}$ NowMattersNow.org, Seattle, WA; ${ }^{4}$ VA Puget Sound Health Care System, Seattle, WA

Background: Firearms are highly lethal, the most common method of suicide death, and receipt of health care prior to suicide death is common. Firearm risk reduction counseling with patients at risk of suicide is a potentially important suicide prevention strategy. However, patient perspectives regarding how questions about firearm access should be asked or implemented in health care settings is unknown. Kaiser Permanente Washington recently implemented a selfadministered monitoring tool for routine depression care across all primary care clinics, which includes a question about firearm access. As part of a broader qualitative interview study focused on understanding patients' experiences of being asked about suicidality during routine primary care, we sought to describe patients' perspectives of the standard question about firearm access.

Methods: Invited patients were sampled using electronic health record data to identify those who had a positive 2-item Patient Health Questionnaire [PHQ-2] screening test for depression at a recent primary care visit. Enrolled participants completed a 15-30-minute semi-structured telephone interview focused on understanding experiences of being asked about suicidality. Interviews (recorded and transcribed) elicited beliefs and descriptions of the experiences of being asked about firearm access at a primary care visit. Thematic domain analysis was used to comprehensively explore responses to the portion of the interview about the firearm question.

Results: A total of 37 men and women, age 20-95 years (mean: 47.6), completed the phone interview. Three organizing themes emerged from qualitative analysis of responses to the part about the firearm question: 1) Respondents believed a standard question about firearm access is appropriate in the context of suicide risk assessment; 2) Respondents believed the question could be improved by clarifying the rationale for asking about firearm access (ie, suicide prevention) and expanding to address other common lethal means; and 3) Respondents were concerned that disclosing access to firearms may result in loss of autonomy and/or compromise of firearm ownership rights.

Conclusion: Clarifying the rationale for asking about firearm access and specifying what will be done with the information may alleviate patients' concerns and optimize honest disclosure. Additional patient perspectives are needed to inform clinical guidelines for and implementation of asking routine questions about firearm access.

OA9.02

Policy Considerations: Systematic Review of Nonpharmacologic Treatment for Chronic Pain

Andrea Skelly, ${ }^{1}$ Roger Chou, ${ }^{2}$ Joe Dettori, ${ }^{3}$ Judith Turner, ${ }^{4}$ Janna Friedly, ${ }^{4}$ Sean Rundell, ${ }^{4}$ Rochelle Fu, ${ }^{2}$ Erika Brodt, ${ }^{1}$ Ngoc Wasson, ${ }^{2}$ Cassandra Winter, ${ }^{1}$ Aaron Ferguson ${ }^{1}$

${ }^{1}$ Aggregate Analytics, Inc., Fircrest, WA; ${ }^{2}$ Oregon Health \& Science University, Portland, OR, ${ }^{3}$ Spectrum Research, Inc., Tacoma, WA; ${ }^{4}$ University of Washington, Seattle, WA

Background: Opioids are commonly utilized for treatment of chronic pain. In 2016, more than 2 million Americans reported struggling with opioid use disorder and an estimated 17,087 overdose deaths involving prescription opioids occurred. Consideration of nonopioid management alternatives, including nonpharmacologic options, and assessment of the evidence for them is vital.

Methods: Randomized controlled trials of noninvasive, nonpharmacologic treatments for 5 common chronic pain conditions (low back pain, neck pain, fibromyalgia, osteoarthritis, and tension headache) that reported results for at least 1 month postintervention were included and critically appraised. Results were summarized for function and pain and focused on persistence of effects.

Results: A total of 202 trials were included. Exercise, multidisciplinary rehabilitation, acupuncture, cognitive behavioral therapy, and mind-body practices were most consistently associated with durable slight to moderate improvements in function and pain for specific chronic pain conditions. Evidence was somewhat more robust for "active" interventions that engage patients in movement and address psychological contributors to pain versus more "passive" treatments focused on symptom relief. Evidence varied across pain conditions. Given variability in patient treatment preferences and differential responses to specific therapies in patients with various chronic pain conditions, policies that broaden access to a broader array of effective nonpharmacologic treatments may have greater impact than those that focus on one or a few therapies. Although the level of supporting evidence varied from condition to condition, we found evidence of persistent effectiveness across conditions for interventions such as exercise, multidisciplinary rehabilitation, psychological interventions, mind-body interventions, and acupuncture.

Conclusion: Our findings provided some support for clinical strategies and policy that focus on use of "active" nonpharmacologic therapies for specific chronic pain conditions. Policy directions might include prioritizing access to interventions with effectiveness across 
conditions, but policymakers need to consider the degree to which evidence may be reasonably extrapolated across conditions. This project was funded under Contract No. HHSA290201500009I from the Agency for Healthcare Research and Quality (AHRQ), U.S. Department of Health and Human Services (HHS). The authors of this abstract are responsible for its content. Statements in the abstract do not necessarily represent the official views of or imply endorsement by AHRQ or HHS.

\section{OA9.03}

Cross-Cultural Measurement Invariance of the Patient Health Questionnaire-9 for American Indian Adults

\section{Melissa Harry, ${ }^{1}$ Stephen Waring ${ }^{1}$}

\section{${ }^{1}$ Essentia Institute of Rural Health, Duluth, MN}

Background: While American Indian and Alaskan Native people have higher rates of suicide compared to other groups, little evidence exists on the prevalence of depression in this population. Scales developed for assessing mental health have been predominately tested in U.S. populations of Western European descent. However, tests of scale reliability and validity should include assessing scale crosscultural invariance (equivalence) between diverse groups, such as through multigroup measurement invariance testing. Establishing cross-cultural measurement invariance, specifically scalar invariance, is required to reliably compare scores between groups. The Patient Health Questionnaire-9 (PHQ-9) is a widely used tool for measuring depression. However, few studies have reported on the measurement invariance of the PHQ-9 for American Indian people. The goal of this study was to help fill that gap by assessing the measurement invariance of the PHQ-9 for American Indian adult patients at Essentia Health, an upper Midwest integrated health care organization.

Methods: Analyses included all American Indian individuals age 18 year and older $(n=4443)$ and a random sample of non-Hispanic white individuals $(\mathrm{n}=4443)$ with a completed PHQ-9 in the electronic medical record at Essentia Health from December 1, 2005, to December 31, 2017. We first performed confirmatory factor analysis with the standard one-factor PHQ-9 model and 5 two-factor models previously identified in the literature separately with American Indian and non-Hispanic white adult patients (age 18-98 years). We then tested nested measurement invariance hypotheses with the best fitting models.

Results: Confirmatory factor analysis showed that the onefactor PHQ-9 model and all 5 two-factor models showed good fits for both groups separately (eg, comparative fit index $>0.95$, root mean square error of approximation $<0.05)$. The one-factor model and best-fitting two-factor models (one with 9 items and another with 7 items) also displayed configural, metric, and scalar invariance between groups.
Conclusion: The PHQ-9 was cross-culturally equivalent in all tested models for American Indian and non-Hispanic white adult individuals, including the standard one-factor model. These results indicate that the PHQ-9 is a suitable depression screening tool for use with American Indian adults in clinical care. However, future research could confirm the generalizability of these findings to other populations of American Indian people.

\section{OA9.04}

Metformin to Manage Side Effects of Psychiatric Medications: Findings From an Integrated Health Care Delivery System

Esti Iturralde, ${ }^{1}$ Boriska Toth, ${ }^{1}$ Lucas Van Dyke, ${ }^{2}$ Roy Eyal, ${ }^{2}$ Jacqueline Kiang, ${ }^{2}$ Linda Kim, ${ }^{2}$ Maggie Mullen, ${ }^{2}$ J. Jewel Shim, ${ }^{2}$ Stacy Sterling ${ }^{1}$

${ }^{1}$ Division of Research, Kaiser Permanente Northern California, Oakland, CA; ${ }^{2}$ The Permanente Medical Group, Oakland, $C A$

Background: More than 10 million adults in the United States have a form of severe mental illness (SMI). Individuals with SMI face elevated risks for diabetes and heart disease, related in part to metabolic side effects of treatment with atypical antipsychotic medications (AAMs). Clinical trial evidence suggests that metformin, an oral diabetes medication, is an effective, well-tolerated treatment for preventing and managing these side effects, but research has not examined the translation of this evidence-based intervention into clinical practice.

Methods: We assessed the prevalence of metabolic risk factors, metformin prescription rates, and predictors of metformin prescriptions among 16,282 adults (age 18-65 years) with a documented SMI diagnosis, no diagnosed diabetes, and a filled AAM prescription during 2015 (index event), using electronic health record data from a large integrated health care delivery system in Northern California.

Results: Participants were of mean age $42.9 \pm 13.7$ years; $59 \%$ were women. The sample was racially diverse $(37.0 \%$ white, 13.6\% Latino, 9.0\% black, 8.6\% Asian, 31.8\% other category). Primary SMI diagnoses were psychotic disorder (29.5\%), bipolar disorder (30.8), other affective disorder (34.1\%), and autism spectrum disorder (4.6\%); $77 \%$ of patients had already-established AAM treatment. Although risk factors for adverse metabolic outcomes were common (eg, 40.4\% with obesity, 78.3\% prescribed an AAM associated with moderate or high risk of metabolic side effects), only 106 patients $(0.65 \%)$ were prescribed metformin during the post-index year. Metformin-prescribing providers often were primary care physicians or psychiatrists, accounting for $50.0 \%$ and $30.2 \%$ of metformin-prescribed patients, respectively. In multilevel logistic regression models (patients nested within AAM prescribers), independent 
predictors of metformin prescription were obesity (odds ratio [OR]: 3.2), female sex (OR: 2.1), primary psychotic disorder (OR: 1.9), established AAM treatment (OR: 1.9), and highmetabolic-risk AAM type (OR: 1.7); all $\mathrm{P}<0.05$. Prescriberlevel effects were significant (intraclass correlation of 0.28 , $\mathrm{P}<0.05$ ), suggesting nonsystematic implementation of metformin treatment.

Conclusion: Many patients with SMI are at elevated risk for adverse cardiometabolic outcomes related in part to their psychiatric medications, but few receive metformin medication, an evidence-based intervention to manage these risks. Future research should focus on strategies for how to overcome implementation barriers.

\section{P12.01}

Predictors of Postdeployment Mental Health Treatment-Seeking Among Iraq and Afghanistan Veterans Seen in Non-VA Facilities

Joseph J. Boscarino, ${ }^{1}$ Yirui $\mathrm{Hu},{ }^{2}$ Thomas Urosevich, ${ }^{2}$ Stuart Hoffman, ${ }^{2} \mathrm{H}$. Lester Kirchner, ${ }^{2}$ Richard Adams, ${ }^{3}$ Ryan Dugan, ${ }^{2}$ Carrie Withey, ${ }^{2}$ Charles Figley, ${ }^{4}$ Joseph A. Boscarino ${ }^{2}$

${ }^{1}$ William James College, Newton, MA; ${ }^{2}$ Geisinger Health, Danville, PA; ${ }^{3}$ Kent State University, Kent, OH; ${ }^{4}$ Tulane University, New Orleans, LA

Background: Currently, there is interest in improving postdeployment mental health treatments among U.S. veterans of Iraq and Afghanistan, yet many veterans do not use the Veterans Affairs (VA) health care system. Consequently, learning about veterans' treatment experiences outside of the VA system is critical for future treatment planning.

Methods: We examined postdeployment mental health treatment among a cross-sectional sample of 235 communitybased veterans of Iraq and Afghanistan receiving care in a large non-VA multihospital system in Pennsylvania.

Results: The mean age of veterans was 42.1 years (standard deviation: 9.2), 87\% were male, and 52\% used the VA in the past year. Among veterans, current posttraumatic stress disorder was $15 \%$ and current depression was $14 \%$. A total of $22 \%$ of participants reported using alcohol/drugs to cope postdeployment, $33 \%$ had a history of concussion, and $16 \%$ reported suicidal thoughts. Overall, $26 \%$ of veterans reported receiving mental health treatment and $27 \%$ reported taking psychotropic medicines in the past year. In multivariable logistic regressions, significant predictors of mental health treatment included female sex (odds ratio [OR]: 5.0, 95\% CI: 1.9-13.1, $\mathrm{P}=0.001)$, current concussion symptoms (OR: 3.0, 95\% CI: 1.4-6.1, $\mathrm{P}=0.003)$, low psychological resilience (OR: 2.7, 95\% CI: 1.4-5.3, $\mathrm{P}=0.005$ ), and using alcohol/ drugs to cope postdeployment (OR: 2.5 , 95\% CI: 1.1-5.4, $\mathrm{P}=0.026)$. The best predictors of recent psychotropic medicine use were VA service use (OR: 4.7, 95\% CI: $2.1-$ 10.5, $\mathrm{P}<0.001$ ), low psychological resilience (OR: 5.3, 95\%
CI: 2.4-11.6, $\mathrm{P}<0.001$ ), current depression (OR: 3.9, 95\% CI: $1.5-10.2, \mathrm{P}=0.005$ ), sleep problems (OR: 2.8, 95\% CI: 1.3-6.0, $\mathrm{P}=0.009)$, and high lifetime trauma exposures (OR: 2.6, 95\% CI: 1.2-5.6, $\mathrm{P}=0.019$ ).

Conclusion: After deployments, both warzone and nonwarzone factors predicted mental health service use among Iraq and Afghanistan veterans seen in non-VA facilities. Further research related to non-VA mental health service use among veterans is advised.

P12.02

Synthesizing Sources for Calculating Morphine Equivalents in Opioid Research

Morgan Fuoco, ${ }^{1}$ Lindsay Jenkins, ${ }^{1}$ Reesa Laws, ${ }^{1}$ Denise Boudreau, ${ }^{2}$ Mary Ann McBurnie ${ }^{1}$

${ }^{1}$ Kaiser Permanente Center for Health Research, Portland, OR; ${ }^{2}$ Kaiser Permanente Washington Health Research Institute, Seattle, WA

Background: The opioid epidemic is a top priority among public health researchers, policymakers, and communities across the United States. Chronic exposure to opioids prescribed for chronic noncancer pain puts a patient at risk for addiction, overdose, and death due to opioids. Clinicians and researchers use morphine equivalents (MEQ) to standardize opioid dose per day in order to assess the extent of exposure and risk, and there are several different methods for computing MEQ from medication-dispensing data. As part of a multisite study evaluating the risk of overdose and death among patients prescribed opioid medications, we developed a strategy for calculating MEQ by synthesizing information across methods.

Methods: We compiled literature and documentation from the most frequently used sources for calculating MEQ. These sources included the most recent conversion factor data file from the Centers for Disease Control and Prevention and the Centers for Medicare \& Medicaid Services, a database from an integrated health system, and published academic articles. Our research team, including a pharmaco-epidemiologist and pharmacist, synthesized information across sources by assessing gaps in MEQ specifications, discrepancies between sources, and overlapping information. We then developed a lookup table for all opioid National Drug Codes (NDC) dispensed during the study period at the 4 participating sites, which included NDC, strength, conversion factor, and reference source. MEQ was calculated by linking the NDC lookup table to dispensing data from the health care systems, which contained NDC, amount, and days' supply.

Results: This approach allowed us to create a comprehensive table of MEQ conversion factors for dispensed opioids that can be applied (either centrally or locally) to opioiddispensing data from multiple health care systems.

Conclusion: By compiling information across sources and engaging subject matter experts, we built a robust, reliable 
data source for calculating MEQ that is transportable to other settings with dispensing data. Having high-quality MEQ data allows researchers to more accurately characterize exposure to opioids and assess risk for dependence, overdose, and death.

\section{$\mathrm{P} 12.03$}

\section{Piloting Specialty Addiction Medicine Consultations in Primary Care via Live, Two-Way Video}

\section{Amy Leibowitz, ${ }^{1}$ Wendy Lu, ${ }^{1}$ Derek Satre,${ }^{1,2}$ Constance Weisner, ${ }^{1}$ Stacy Sterling ${ }^{1}$ \\ ${ }^{1}$ Division of Research, Kaiser Permanente Northern California, Oakland, CA; ${ }^{2}$ Weill Institute for Neurosciences, University of California, San Francisco, San Francisco, CA}

Background: Screening, brief intervention, and referral to treatment (SBIRT) is known to help primary care patients reduce unhealthy alcohol use, but less is known about whether it helps patients with more severe problems connect to appropriate treatment. SBIRT holds promise for facilitating successful treatment initiation, but few interventions have addressed the challenges in doing this. We piloted an on-call service whereby specialty addiction medicine consultants provided real-time video consultation - including motivational interviewing and information about medication-assisted treatment - to patients presenting in primary care with likely alcohol use disorders (AUDs).

Methods: "Alcohol as a Vital Sign 2.0: Addiction Medicine Video Consultation" was a 1-year feasibility pilot conducted in adult primary care in a large Kaiser Permanente Northern California (KPNC) medical center. Researchers developed and implemented video consult workflows, continuing medical education (CME) trainings, an on-call staffing protocol, and ongoing technical assistance. Observational data included CME attendance and evaluations, consult service utilization rates, and technological challenges encountered. Qualitative data included interviews with key stakeholders and results of a user survey. Preliminary data about patient outcomes, including specialty and medicationassisted treatment initiation, were extracted from KPNC electronic health records.

Results: A total of 91 primary care physicians (79\%) attended a CME training, and $98 \%$ rated it somewhat or very useful; 33 physicians ( $26 \%$ ) requested a total of 52 consults, and specialists provided video consults for 32 primary care patients (62\% success rate). Challenges included consultant unavailability, technology problems, and time constraints. Physicians rated the service as valuable (average of 6.9 on a scale for which $1=$ not valuable and $10=$ extremely valuable), and almost all (17 of 18 respondents) indicated they would use the service again if technology and staffing issues were solved. Of patients receiving a video consult, 12 $(38 \%)$ initiated treatment (defined as attending $1+$ addiction medicine appointment within 14 days of the consult) and 11
(34.4\%) were prescribed an AUD medication.

Conclusion: Utilization rates and stakeholder feedback suggest that it is feasible to implement a larger-scale, realtime addiction medicine video consult service and that such a centralized resource holds promise for improving AUD treatment initiation rates.

\section{P12.04 \\ Demographic, Clinical, and Prescribing Characteristics Associated With Future Opioid Use in an Opioid-Naïve Population}

\section{Gabriela Rosales, ${ }^{1}$ Weiming $\mathrm{Hu},{ }^{1}$ Rajasekhara Mummadi, ${ }^{2}$ Jose Benuzillo, ${ }^{1}$ David Mosen ${ }^{3}$ \\ ${ }^{1}$ Kaiser Permanente Center for Health Research, Portland, OR; ${ }^{2}$ Northwest Permanente, P.C., Portland, OR; ${ }^{3}$ Kaiser Permanente Northwest, Portland, OR}

Background: Opioid prescriptions in the United States have nearly tripled since 1991 . To reduce opioid utilization, research is needed to identify risk factors associated with future opioid use, especially among those with no prior use (naïve users). The study objective was to examine demographic, clinical, and prescribing characteristics associated with future opioid use in this population.

Methods: We analyzed the electronic records of Kaiser Permanente Northwest members with 1 or more opioid dispenses from January 1, 2010, to September 30, 2015, who did not have a dispense in the previous 6 months $(n=23,804)$. The outcome measure was receipt of a third dispense of opioids after index dispense. Demographic, clinical, and prescribing characteristics were age (46-65 vs $>65$ vs $\leq 45$ years), race (white vs nonwhite), ethnicity (Hispanic vs nonHispanic), Charlson comorbidity index (CCI of $1+$ vs 0$)$, any prior substance-abuse diagnosis (yes vs no), any prior mental health diagnosis (yes vs no), prescribing location (hospital, outpatient vs other), opioid duration (extended-release vs short-release) and morphine milligram equivalency (MME of $\geq 90$ vs $<90$ ). Multivariable logistic regression was used to the measure the associations between these variables and receipt of a third dispense of opioids.

Results: We found a higher likelihood of a third opioid dispense with the following: age 45-64 years (odds ratio [OR]: 1.34, 95\% CI: 1.25-1.44); age >65 years (OR: 1.23, 95\% CI: 1.13-1.33), white race (OR: $1.24,95 \%$ CI: $1.11-$ 1.39), CCI of $\geq 1$ (OR: $1.14,95 \%$ CI: 1.07-1.21), prior substance abuse (OR: 1.19, 95\% CI: 1.11-1.27), prior mental health diagnosis (OR: 1.25, 95\% CI: 1.18-1.33), extended/ long-release duration (OR: 1.53, 95\% CI: 1.43-1.64), and MME of $\geq 90$ (OR: 3.00, 95\% CI: 2.80-3.21). Two factors were associated with a lower likelihood of having a third opioid dispense: Hispanic ethnicity (OR: 0.60, 95\% CI: $0.52-0.69$ ) and receipt of a prescription for medication in a hospital setting (OR: 0.20, 95\% CI: 0.17-0.22).

Conclusion: Several characteristics were associated with 
future opioid use. Of interest, members whose prescriptions had an MME of $\geq 90$ at index dispense were 3 times more likely to have a third dispense of opioids compared to MME of $<90$. Future work is needed to understand how to employ prescribing and other characteristics to develop predictive risk tools that can be implemented to reduce opioid use.

\section{$\mathrm{P} 12.05$}

\section{Navigating Practical, Legal, and IT Challenges to Bring Assessment and Treatment of Pediatric ADHD in Primary Care at Geisinger into the 21st Century}

Sean O'Dell, ${ }^{1}$ Alanna Rahm, ${ }^{1}$ Amanda Young, ${ }^{1}$ Paul Kettlewell, ${ }^{1}$ Amy Kolinovsky, ${ }^{1}$ Hiyan Sun, ${ }^{1}$ Tracey Klinger, ${ }^{1}$ Rachel Kininger, ${ }^{1}$ Christine Lee, ${ }^{1}$ Kathy Dehart $^{1}$

\section{${ }^{1}$ Geisinger Health, Danville, $P A$}

Background: Although families and schools often rely on primary care physicians (PCPs) to assess and treat attentiondeficit/hyperactivity disorder (ADHD), PCPs often go without the necessary resources to make clinical decisions based on validated screening tools for parents and teachers implemented through the electronic health records (EHR). Prior research addressing this problem is limited by lengthy initial trainings that are infeasible in real-world settings, proprietary fee-per-administration screening tools, and lack of population-based clinical outcome data. The current project developed and tested a rollout of tools to improve PCPs adherence to American Academy of Pediatrics (AAP) guidelines for the assessment and treatment of ADHD.

Methods: All Geisinger providers in pediatrics sites who assess and treat pediatric ADHD were approached for consent. All clinics with at least one consented PCP were cluster randomized at the clinic level to intervention or wait-list control, stratified by number of new ADHD patients at the clinic in the last year and presence of an onsite behavioral health provider. The PRISM model (2008) informed stakeholder interviews with site leads and medical directors to develop intervention content. HIPAA/FERPAcompliant electronic Vanderbilt ADHD Rating Scales for parents and teachers were developed. Survey results are routed to the PCP for review; documentation templates and best practice alerts facilitate follow-up assessments on the AAP-recommended timeline. Informed by Moore's behavior change principles (2009), the initial 1-hour in-person training on AAP guidelines and EHR tools are supplemented by 1-, 3-, and 6-month performance support consultations. Evaluation of provider satisfaction, declarative knowledge, self-efficacy, intention to change, and perceived barriers will be assessed pre/post training and 6-month follow-up. PCP clinical performance will be assessed using interrupted time series analysis on key quality indicators extracted from the EHR or abstracted through chart review.
Results: 21 sites (75\%) had at least one PCP consent for participation. Overall, the participation rate for PCPs was 78\%. Trainings commenced in late fall 2018 and follow newly diagnosed patients for a 1-year post-training period.

Conclusion: We were able to engage a substantial proportion of the intended population in the intervention. Results from the pre/post training evaluation will be discussed as well as detailed description of implementation strategies and analysis of key quality indicators.

\section{P12.07 \\ Better Outcomes, Best Care: Learning From Every Patient to Improve Outcomes}

\section{Rachel Solomon, ${ }^{1}$ Paul Kurdyak, ${ }^{1}$ Jennifer Wilkie ${ }^{1}$ \\ ${ }^{1}$ Centre for Addiction and Mental Health, Toronto, Canada}

Background: When it comes to physical health, we would never treat a diabetes patient without measuring their glucose levels and knowing how they respond to treatment. Measuring how different groups of patients respond to different treatments is pivotal to planning and providing care that ensures the best outcomes for patients. However, every day in the mental health system, patients receive care that is neither standardized nor measurement-based, often leading to poor patient outcomes.

Methods: The Centre for Addiction and Mental Health $(\mathrm{CAMH})$ is the largest academic mental health hospital in Canada and one of the world's leading mental health research centers. In 2018, CAMH launched the Better Outcomes, Best Care initiative. The objective of the hospitalwide initiative is to achieve: a) Gaps are systematically identified in access and quality of care using provincial and CAMH data; b) Research will have a patient-oriented focus by engaging patients as partners and focusing on patient-identified priorities; c) Solutions can be designed and implemented using a variety of methods, including clinical trials, Lean, implementation science, and quality improvement (QI) methods; and d) Everything from randomized control trials, QI implementation, and complex intervention design can be used to evaluate interventions. The methodology for implementation was to leverage existing and wellestablished learning health systems in acute care settings to apply the foundational requirements of these established models to a mental health setting.

Results: Significant capstone projects have been established and are producing results, shifting the culture to one of continuous measurement and improvement. Preliminary results include an evidence-based multipronged approach to improving discharge processes and outcomes across the hospital, implementation of a single functional outcome measure across the hospital using the World Health Organization Disability Assessment Schedule, and streamlining of clinical assessment tools and measures to create a minimum data set for outcome measurement. 
Conclusion: Better Outcomes, Best Care is the beginning of a transformation, not only within CAMH but across the mental health system. Early implementation results signal the power of the learning health system model in transforming mental health service delivery, but also the seismic shift it represents and the importance of a large-scale commitment to the change.

\section{P12.08}

\section{Adolescent SBIRT: Health Care Utilization and Comorbid Problems Over 3 Years}

\section{Stacy Sterling, ${ }^{1}$ Andrea Kline-Simon, ${ }^{1}$ Ashley Jones, ${ }^{1}$ Lauren Hartman, ${ }^{2}$ Katrina Saba, ${ }^{2}$ Sujaya Parthasarathy ${ }^{1}$ \\ 'Division of Research, Kaiser Permanente Northern California, Oakland, CA; ${ }^{2}$ Pediatrics, The Permanente Medical Group, Oakland, CA}

Background: Little is known about the effects of screening, brief intervention, and referral to treatment (SBIRT) on health care utilization among adolescents. We describe health care utilization findings from a trial of SBIRT in pediatric primary care.

Methods: We randomized clinic pediatricians $(n=52)$ to 3 study arms: 1) pediatrician-only (PCP), in which they were trained to deliver SBIRT; 2) embedded-BHC, in which they could refer patients to a behavioral health clinician (BHC); and 3) usual care (UC). We used electronic health record data to obtain all inpatient and outpatient utilization (emergency department [ED], primary care, psychiatry, and chemical dependency) during the 3 years following the index visit, across the 3 arms. We used logistic regression to examine any ED or inpatient use and negative binomials. The exponent of the estimated coefficient represents the odds ratio or rate ratio, with a value less than 1 indicating less likelihood of a visit (for logistic) or fewer visits (for negative binomial distribution of primary care, psychiatry, chemical dependency, and total outpatient visit counts).

Results: There were 1871 eligible patients. At 1 year postindex, there were no differences in primary care or inpatient utilization between the arms. Compared to UC, adolescents in the BHC arm had less likelihood of ED use (adjusted odds ratio [aOR]: 0.60, 95\% CI: 0.34-1.05) and were likely to have fewer psychiatry visits (adjusted rate ratio [aRR]: $0.73,95 \% \mathrm{CI}: 0.52-1.02$ ) or any outpatient utilization (aRR: 0.67, 95\% CI: 0.51-0.87). Adolescents in the PCP arm also had less likelihood of ED use than those in UC (aOR]: 0.76, 95\% CI: 0.59-0.98). At 3 years postindex, there were no differences in the likelihood of primary care, inpatient, or ED utilization across the arms. However, compared to those in the UC arm, adolescents in the BHC and PCP arms had fewer psychiatry visits (aRR for BHC: 0.56, 95\% CI: 0.41-0.76; aRR for PCP: 0.73, 95\% CI: $0.53-0.99$ ) or any outpatient services (aRR for
BHC: $0.76,95 \%$ CI: 0.64-0.91; aRR for PCP: $0.78,95 \%$ CI: 0.65-0.93).

Conclusion: Adolescents in both intervention arms were less likely to use several types of health care, including ED services. SBIRT, delivered as part of pediatric primary care, may obviate the need for more costly specialty and emergency care.

P12.09

Pharmacoeconomic Evaluation of Naloxone Distribution for the Prevention of Opioid-Overdose Fatalities: A Systematic Review

\author{
Toney Duong, ${ }^{1}$ Anh Thu Tran, ${ }^{1}$ Joy Alonzo, ${ }^{1}$ Lixian \\ Zhong ${ }^{1}$
}

\section{${ }^{1}$ Texas A\&M University, College Station, $T X$}

Background: Drug-overdose mortality has become the number one cause of accidental deaths in the United States. In 2017, the Department of Health and Human Services declared the opioid epidemic a public health emergency. Naloxone, an opioid-overdose reversal agent, was recommended by the surgeon general to reduce opioidoverdose deaths. This study aimed to evaluate the costeffectiveness of naloxone distribution to adults at risk of opioid overdose via a systematic literature review.

Methods: We conducted a systematic review following the Preferred Reporting Items for Systematic Review and Metaanalysis (PRISMA) statement. MEDLINE/PubMed and Embase were searched using key terms to identify primary sourced articles published between January 1997 and June 2018. Two independent reviewers searched evaluated studies based on inclusion/exclusion criteria and performed data extraction.

Results: We identified 5 studies, including 4 costeffectiveness studies and 1 cost-benefit study. The studies were done in different countries and different health care settings, providing a broad assessment of different scenarios. All cost-effectiveness studies reported low incremental costeffectiveness ratios ranging from $\$ 94$ to $£ 899$ (or $\sim \$ 1185$ ) per quality-adjusted life-year (QALY) gained, suggesting that distributing naloxone to at-risk population costs relatively little to gain additional QALYs. In addition, the cost-benefit analysis suggested that such programs can be cost-saving, as the naloxone drug acquisition cost was offset by mean medical resource savings of about $\$ 472,000$ in 2015.

Conclusion: Naloxone distribution to heroin users for lay overdose reversal is cost-effective at standard willingnessto-pay of $\$ 50,000-\$ 100,000$ in U.S. dollars or $£ 20,000$ in U.K. pounds. Naloxone acquisition costs are offset by medical cost reductions. Our study synthesized existing pharmacoeconomic evidence on naloxone distribution to atrisk populations, facilitating the planning of such programs in the future. 


\section{CARE IMPROVEMENT}

OA3.01

Dissemination and Sustained Use of an Outpatient Clinical Decision System With High Use Rates, High Clinician Satisfaction, and Positive Impact on Quality of Care

JoAnn Sperl-Hillen, ${ }^{1}$ A. Lauren Crain, ${ }^{1}$ Jay Desai, ${ }^{1}$ Karen L. Margolis, ${ }^{1}$ Heidi L. Ekstrom, ${ }^{1}$ Deepika Appana, ${ }^{1}$ Christina Wood, ${ }^{1}$ Rashmi Sharma, ${ }^{1}$ Patrick J. O'Connor ${ }^{1}$

\section{${ }^{1}$ HealthPartners Institute, Bloomington, $M N$}

Background: We describe the performance of an electronic health record (EHR)-linked, web-based primary care cardiovascular clinical decision support (CDS) system and the facilitators and barriers to dissemination.

Methods: Algorithms identify patients at the point-of-care and target them to receive personalized and prioritized evidence-based treatment options. Printed versions of the CDS for patients and providers are used to elicit treatment preferences and facilitate shared decision-making. In 3 National Institutes of Health (NIH)-funded clusterrandomized trials, the CDS use ranged from $71 \%-77 \%$ of targeted patients, and clinicians reported 85\%-98\% satisfaction. Blood pressure control, glucose control, and 10-year cardiovascular risk trajectory significantly improved in patients cared for at clinics randomized to CDS vs usual care.

Results: This CDS system has been disseminated to 4 large care delivery systems with about 2000 primary care clinicians in 4 states, with dissemination to an additional 60 safety-net clinics in 10 states underway. The CDS website currently evaluates 40,000 visits a day ( 9 million visits per year) with high reliability and 300 millisecond/ case processing time. Facilitators of dissemination have included care system or payer interest in care improvement, clinician satisfaction, high CDS use rates with minimal impact on workflow, favorable effects on patient outcomes and shared decision-making, positive patient perceptions, standardization of care, and ability to rapidly translate new evidence/guidelines into practice. Barriers to dissemination include cost of implementation and lack of a corporate entity to market and manage ongoing support of the CDS system (not a strength of a research organization with limited ability or bandwidth to devote to this). Actual implementation costs per delivery system were about $\$ 100,000$, mostly related to effort required for data mapping and programming the interconnectivity between EHR and the web service. Time required for implementation was about 4-6 months after data and service agreements were finalized and varied depending on perceived priority of the project and skill level of programmers at the recipient organization.

Conclusion: This CDS system had high use rates, high primary care provider satisfaction, and significantly improved care in targeted adults and children. After 10 years of NIHfunded development and expansion, several potentially remediable factors limit widespread dissemination.

\section{OA3.02 \\ Challenges Encountered in Linking Community Health Centers' Electronic Health Record Data to a Web-Based Clinical Decision Support Tool}

Mary Middendorf, ${ }^{1}$ Marla Dearing, ${ }^{1}$ Rachel Gold, ${ }^{1,2}$ JoAnn Sperl-Hillen, ${ }^{3}$ Patrick J. O'Connor, ${ }^{3}$ Erik Geissal, ${ }^{1}$ John Heintzman, ${ }^{1,4}$ Stuart Cowburn, ${ }^{1}$ Arwen Bunce, ${ }^{1}$ Deepika Appana, ${ }^{3}$ Christina Wood, ${ }^{3}$ Rashmi Sharma, ${ }^{3}$ M.J. Dunne ${ }^{1}$

${ }^{1}$ OCHIN, Portland, OR; ${ }^{2}$ Kaiser Permanente Center for Health Research, Portland, OR; ${ }^{3}$ HealthPartners Institute, Minneapolis, MN; ${ }^{4}$ Oregon Health \& Science University, Portland, OR

Background: Clinical decision support (CDS) tools, which process patient data via algorithms based on up-to-date evidence, can help disseminate care guidelines by keeping providers abreast of current recommendations. However, such CDS tools are often available only in the delivery system or electronic health record (EHR) where they were developed, necessitating reprogramming at multiple sites when care guidelines change. This inefficiency could be addressed if data from any EHR could be sent to a website with regularly updated CDS algorithms. We are currently testing the effectiveness of CV Wizard ${ }^{\circ}-$ a web-based CDS tool run by HealthPartners - in 68 community health centers (CHCs) that share an EHR through OCHIN.

Methods: Between March 2017 and April 2018, OCHIN and HealthPartners collaborated to establish a data exchange between OCHIN's Epic EHR and the CV Wizard CDS website. Needed EHR data (eg, demographics, diagnoses, labs, allergies, vitals) were sent to CV Wizard, which then sent CDS suggestions back to the EHR.

Results: Numerous challenges in establishing this data exchange were identified and resolved. These included determining: who maintains records of data sent/responses sent back; what legal agreements were required for needed data retention; the method for sending CDS results back to the EHR; whether to send all available medication/laboratory/ allergy/diagnosis data to the CDS or just data points currently needed by the algorithm; how to manage security concerns related to the transmission of real-time patient data; whether the CDS' cache routine could be modified to improve performance/sustainability; how OCHIN's unique structure (a collaborative of many independent organizations) necessitated rethinking managing data from more than 60 lab interfaces; how to generate data on CDS use rates; and how to modify the CDS tool's "feedback" function for consistency with OCHIN's usual support practices. Barriers to customizing the cardiovascular disease risk cut-point that 
triggered the CDS tool also were identified.

Conclusion: Inefficiencies are inherent when new care guidelines must be programmed in myriad CDS systems. We demonstrated that multiple EHRs can successfully plug into a single CDS algorithm, after resolution of multiple data exchange challenges.

\section{OA3.03}

\section{The Impact of a Web-Based Point-of-Care Tool on Physician Behavior and Patient Health Outcomes}

\author{
J.B. Jones, ${ }^{1}$ Xiaowei Yan, ${ }^{1}$ Hannah Husby, ${ }^{1}$ Jake \\ Delatorre-Reimer, ${ }^{1}$ Farah Refai, ${ }^{1}$ Shruti Vaidya, ${ }^{1}$ \\ Ridhima Nerlekar, ${ }^{1}$ Karen MacDonald ${ }^{2}$
}

${ }^{I}$ Sutter Health, Walnut Creek, CA; ${ }^{2}$ AstraZeneca, Wilmington, $D E$

Background: Primary care physicians face various challenges, including increasing numbers of patients with cardiometabolic conditions (diabetes, hypertension, dyslipidemia), growing patient panel sizes, more administrative tasks, lack of user-friendly resources, and an ever-increasing knowledge in medicine, which makes managing patients' cardiometabolic conditions more challenging. In collaboration with AstraZeneca, Sutter Health has developed CM-SHARE, a web-based point-ofcare tool for primary care physicians that aims to display targeted information for providers to use to engage and manage their patients while also reducing the amount of time spent on the computer searching for information.

Methods: CM-SHARE was piloted at 2 primary sites in Northern California with 6 primary care providers. Application usage data were tracked, and one-on-one interviews with users were conducted to understand user adoption. Based on electronic health record (EHR), a pre/ post parallel, matched-control longitudinal analysis of clinical outcomes and workflow process measures was conducted to understand CM-SHARE's impact on EHR usage, clinic workflow, provider care delivery, and patient clinical outcomes. Comparisons were made using t-tests and Wilcoxon signed-rank test.

Results: Over 26 months since deployment, pilot providers used CM-SHARE in $33 \%(\mathrm{~N}=37,131)$ of all patient encounters. EHR and pilot user feedback indicate that CMSHARE was most used for complex high-morbidity patients with cardiometabolic conditions, particularly in diabetesfocused visits (53\% vs $28 \%$ usage for all encounters). Physicians reported high value in CM-SHARE's ability to visualize health data and to educate high-morbidity patients. Use of CM-SHARE was associated with a $25 \%-$ $35 \%$ reduction in EHR time and 11\%-20\% fewer EHR clicks for cardiometabolic-focused encounters. Compared with control patients, patients with diabetes who had CMSHARE launched had, on average, more hemoglobin A1c labs ordered $(\mathrm{P}=0.0001$ and $\mathrm{P}=0.0007$ at 6 -month and 12-month follow-up periods, respectively) and achieved a $0.1 \%$ lower hemoglobin $\mathrm{A} 1 \mathrm{c}$ lab value $(\mathrm{P}=0.02$ at 18 -month follow-up).

Conclusion: CM-SHARE has maintained stable usage over the course of the pilot, indicating that it fulfills a valuable physician need. Early results indicate positive trends in both reduction of EHR burden and improvement of disease monitoring and patient clinical outcomes. More work is needed to understand how CM-SHARE mediates patient outcomes and physician behavior change.

OA3.04

Transforming Hepatitis B Care in Kaiser Permanente Mid-Atlantic States Through a Registry and Coordinator-Supported Pathway

\section{Cabell Jonas, ${ }^{1}$ Michael Horberg ${ }^{1}$}

${ }^{1}$ Mid-Atlantic Permanente Research Institute, Rockville, MD

Background: Chronic hepatitis B (HBV) is a viral disease causing significant morbidity and mortality through cirrhosis and liver cancers such as hepatocellular carcinoma. Liver cancer is the 5th and 8th leading cause of cancer deaths among U.S. males and females, respectively. In developing a hepatocellular carcinoma surveillance program, the MidAtlantic Permanente Medical Group (MAPMG) uncovered upstream gaps in HBV diagnosis and documentation, which were influencing hepatocellular carcinoma surveillance eligibility. In response, MAPMG created a technologybased and coordinator-supported Hepatitis B Pathway to update HBV diagnoses among patients new to Kaiser Permanente Mid-Atlantic States, patients who have never seen a MAPMG gastroenterologist for HBV, and patients who have not seen a MAPMG gastroenterologist for HBV in $2+$ years.

Methods: The Hepatitis B Pathway uses a "back end" EMRembedded registry-based patient identification process and a "front end" initiating order that enables providers to directly refer patients. By using two access points, the program can conduct proactive outreach and receive direct referrals from physicians. Eligible patients also are identified through gastroenterology chart reviews. Coordinators utilize an Epic Reporting Workbench workflow tool to track patients through each step of the Hepatitis B Pathway. The structure of the patient registry, orders, and workflow tools are relevant to other Health Care Systems Research Network members planning population health management programs.

Results: Patients receive lab tests related to hepatitis B, liver function, and co-infections as well as an abdominal ultrasound. Patients have a gastroenterology encounter (primarily by telephone) to review results, and hepatocellular carcinoma surveillance eligibility is documented within the chart. Between December 2017 and September 2018, 1329 patients participated. Preliminary data will be presented.

Conclusion: The Hepatitis B Pathway has several aspects 
that can inform the design of population health management programs. These include registry construction, the Reporting Workbench tool, lessons learned about registry-based vs order-based access to the Pathway, performance metrics, etc. This program serves as an example of how clinical, operational, and research partnerships can effectively improve clinical care.

\section{OA11.01}

\section{Communicating Personalized Diabetes Risk With Options for Weight Management: Recruitment, Enrollment, and Outcomes}

Craig Wood, ${ }^{1}$ Lisa Bailey-Davis, ${ }^{1}$ Jacob Mowery, ${ }^{1}$ Christopher Seiler, ${ }^{1}$ Adam Cook, ${ }^{1}$ Krystal Cunningham, ${ }^{1}$ Allison Naylor, ${ }^{1}$ Scott Jamieson, ${ }^{1}$ David Rolston, ${ }^{1}$ Christopher Still ${ }^{1}$

\section{${ }^{1}$ Geisinger Health, Danville, $P A$}

Background: Primary care providers (PCPs) should offer or refer individuals with obesity or impaired/abnormal glucose to intensive multicomponent behavioral intervention. However, $<33 \%$ receive weight management advice, $<5 \%$ are referred for treatment, and only $15 \%$ would pursue weight management based on a PCP recommendation alone. Patients may be more likely to pursue weight management if they believe weight loss will improve future health. A personalized diabetes risk calculator was developed to assess the impact of weight loss on future diabetes incidence. The goal of this study was to determine if a diabetes risk calculator (DRC) can be implemented with PCPs, result in enrollment into effective weight management programs, and improve diabetes health indicators.

Methods: Adult patients $(\mathrm{N}=338)$ with body mass index $(\mathrm{BMI}) \geq 27 \mathrm{~kg} / \mathrm{m}^{2}$ and prediabetes (hemoglobin A1c: $5.7 \%-6.4 \%$ ) received a letter describing their individual risk of developing diabetes within 3 years and corresponding reductions in risk with weight loss of $5 \%, 10 \%$, and $15 \%$. Interested patients were invited to select a 6-month weight management treatment from the following options: remote telephone counseling with a dietician, Fitbit, and BMIQ website portal (RD); exercise class utilizing the Diabetes Prevention Program (DPP); Weight Watchers (WW); pharmacologic treatment plus RD (MEDS+RD); or pharmacologic treatment plus DPP (MEDS+DPP). Preliminary 6-month outcomes are reported.

Results: The recruitment response rate was $25 \%(\mathrm{n}=84$; $52 \%$ female; mean age: 57 years). The most popular treatment selection was MEDS+RD (33\%), followed by RD (31\%), DPP (15\%), WW (11\%), and MEDS+DPP (10\%). Of the first 67 patients who initiated the 6-month program, $79 \%$ completed treatment. Six-month data collection was completed for 13 patients: mean weight loss was $8.4 \%$ (standard deviation [SD]: 5.3, $\mathrm{P}<0.0001$ ) - including $85 \%$ with $>5 \%$ loss of baseline weight - and mean hemoglobin
Alc decrease was 0.2 (SD: $0.2, \mathrm{P}=0.0018$ ). When applying these results to the $\mathrm{DRC}$, the mean risk of 3-year diabetes decreased from $24.1 \%$ to $11.6 \%$ ( $\mathrm{P}=0.0017$ ).

Conclusion: The use of a personalized DRC to motivate weight management resulted in a high recruitment rate and low attrition, leading to meaningful weight loss and a reduction in hemoglobin A1c. Personalized risk letters are a feasible means for informing patients of their risk of developing diabetes and facilitating enrollment in available treatment programs.

\section{OA11.02 \\ Predictors of Opioid Tapering Rate: FLOAT Study}

David H. Smith, ${ }^{1}$ Jennifer Kuntz,, Jennifer L. Schneider, ${ }^{1}$ Alison Firemark, ${ }^{1}$ John Dickerson, ${ }^{1}$ Xiuhai Yang, ${ }^{1}$ Dea Papajorgji-Taylor, ${ }^{1}$ Mark Sullivan, ${ }^{2}$ Lynn DeBar, ${ }^{3}$ Lou Ann Thorsness, ${ }^{4}$ Katherine Reese ${ }^{4}$

${ }^{1}$ Kaiser Permanente Center for Health Research, Portland, OR; ${ }^{2}$ University of Washington, Seattle, WA; ${ }^{3}$ Kaiser Permanente Washington Health Research Institute, Seattle, WA; ${ }^{4}$ Clinical Pharmacy Services, Kaiser Permanente Northwest, Portland, OR

Background: Reducing opioid use is a high priority; however, little is known about patient characteristics associated with tapering. Our objective was to identify predictors of tapering rate among patients referred to a pharmacist-led opioid tapering program.

Methods: We assembled a retrospective cohort of adult $(>20$ years of age) Kaiser Permanente Northwest patients using long-term, high-dose opioids and referred to a pharmacistled opioid tapering service between February 1, 2010, and December 31, 2016. Time zero was the first contact with the program; patients were required to have continuous membership and drug coverage during the baseline year. The outcome was the rate of change in milligram morphine equivalents per day (MMED) each month over the year following index. Candidate predictors were identified a priori based on published literature and clinician input and were measured during the baseline year using Kaiser Permanente Northwest's electronic health record. Predictors included age, sex, socioeconomic status (SES), smoking, body mass index (BMI), MMED, comorbid conditions (mental health, type of pain diagnosis), health care utilization, and baseline opioid trajectory. Generalized estimating equations estimated the monthly change in MMED for each predictor, controlling for concomitant medications; significance was defined as alpha $<0.05$.

Results: We included 1424 patients who were referred for tapering. Patient's average age was 57 years; $62 \%$ were female. Patient's MMED were high $(>200)$ and stable at baseline. Controlling for baseline medication use, faster tapering (negative coefficient) was associated with nonwhite patients (monthly MMED change: $-1.07,95 \%$ CI: -1.97 to 
-0.16), lower SES (-0.77, 95\% CI: -1.48 to -0.06$)$, lower BMI (-1.50, 95\% CI: -2.25 to -0.74$)$, more emergency department $(-0.16,95 \% \mathrm{CI}:-0.31$ to -0.02$)$ and urgent care visits $(-0.02,95 \% \mathrm{CI}:-0.04$ to -0.01$)$, higher comorbidity $(-0.30,95 \%$ CI: -0.45 to -0.16$)$, and highest baseline opioid trajectory $(-1.25,95 \% \mathrm{CI}:-2.14$ to -0.35$)$. Slower tapering (positive coefficient) was associated with smoking (monthly MMED change: +0.82 , 95\% CI: 0.16 to 1.47 ), pain group visits $(+0.47,95 \% \mathrm{CI}: 0.30$ to 0.64$)$, more hospital stays $(+0.87,95 \% \mathrm{CI}: 0.46$ to 1.27$)$, diagnoses of depression $(+1.14,95 \%$ CI: 0.58 to 1.71$)$, fibromyalgia $(+0.68,95 \% \mathrm{CI}$ : 0.11 to 1.25$)$, more unique pain diagnoses $(+0.37,95 \% \mathrm{CI}$ : 0.19 to 0.55$)$, and lowest baseline opioid trajectory $(+0.63$, 95\% CI: 0.01 to 1.25$)$.

Conclusion: These data may help in designing tapering interventions, including setting expectations for patients and clinicians and identifying patients needing greater resource intensity (eg, mental health consult) to successfully taper.

\section{OA11.03}

\section{Proton-Pump Inhibitor Treatment Patterns Among Israeli Adults, 2000-2015}

Vered Rosenberg, ${ }^{1}$ Gabriel Chodick, ${ }^{1}$ Clara Weil, ${ }^{1}$ Varda Shalev, ${ }^{1}$ Kariv Revital ${ }^{1}$

\section{${ }^{I}$ Maccabi Healthcare Services, Tel-Aviv, Israel}

Background: Proton-pump inhibitors (PPIs) are a leading effective therapy for acid-related disorders. Several studies have found that PPIs are overused in ambulatory care settings. The current study aims to describe patterns and duration of PPI treatment in a real-world setting.

Methods: We conducted an observational study using the computerized database of Maccabi Healthcare Services. Study population included adult members who first purchased PPIs between January 1, 2000, and December 31, 2015. Annual incidence and prevalence rates were calculated. The total annual PPI consumption was measured using defined daily dose (DDD). Indications for initiating PPI treatment were defined according to gastrointestinal-related diagnoses and dispensed medications. Duration of PPI treatment, up to 5 years after the first purchase, was assessed using the proportion of patients covered (PPC) method.

Results: Study population included 528,420 patients. The mean annual incidence rate was 2780.4 (standard deviation: $202.9)$ per 100,000 . The annual prevalence rate increased steadily, from 2360.9 per 100,000 in 2000 to 12,683 in 2015. The total annual consumption increased from 1.3 million DDDs in 2000 to 34.7 million in 2015. At least one gastrointestinal-related diagnosis was available at treatment initiation for 325,786 patients $(62 \%)$. The most common diagnoses were signs, symptoms, and functional disorders $(116,421$ [22\%]). Approximately $15 \%$ of all patients maintained PPI therapy in the 5 years of follow-up. In the subgroup analyses, older age and diagnosis of reflux disease were associated with longer duration of treatment, as measured by proportion of patients covered in the 5 years after starting PPI treatment (18-29 years of age: $5 \%$ vs $\geq 60$ years of age: $27 \%-41 \%$; reflux disease: $20 \%$; and $H$. Pylori infection: $10 \%$ ). Interestingly, approximately $15 \%$ of those who had no record of any gastrointestinal-related diagnosis maintained PPI therapy in the 5 years of follow-up.

Conclusion: Despite potential risks and current recommendations to minimize long-term use, there is evidence for PPI overuse. The prevalence rate of PPI use increased steadily, often with no apparent clinical indication. Further examination of indications for continued PPI treatment is advised, especially among those $\geq 60$ years old who are more likely to be treated for longer periods.

\section{OA11.04 \\ Reducing Medical Overuse Across Three Diverse Health Care Settings: Lessons Learned}

\author{
Michael Parchman, ${ }^{1}$ Lorella Palazzo, ${ }^{1}$ Gabrielle \\ Gundersen, ${ }^{1}$ Paula Blasi, ${ }^{1}$ Nora Henrikson, ${ }^{1}$ Brian \\ Austin $^{1}$
}

${ }^{1}$ Kaiser Permanente Washington Health Research Institute, Seattle, WA

Background: Health care organizations frequently struggle to reduce the use of services for which the risk of harm exceeds the possible benefit. Herein, we describe findings from 3 diverse health care organizations that applied the Taking Action on Overuse Framework to address overuse. Over 15 months, study team members and external facilitators supported each organization's efforts to engage providers, patients, and care teams in an overuse-reduction quality improvement initiative. A large hospital system addressed overuse of the emergency department, an academic specialty department addressed overuse of endoscopy, and a statewide primary care association addressed opioid overprescribing in community health clinics.

Methods: Stakeholders from each organization described experiences with their overuse-reduction initiative during midpoint phone interviews and during a 2-day capstone meeting at the end of the project. The team reviewed interview transcripts and capstone meeting notes, identified common themes and lessons learned, and triangulated findings with observations from external facilitators and study team members. We were particularly interested in understanding how their work and experience with reducing overuse compared to traditional quality improvement.

Results: Sites chose their overuse-reduction topic based on both fit with organizational strategic priorities and the prevalence of overuse; 15 months into the initiatives, all sites had engaged with leadership and gathered data to frame their overuse problem. None had fully engaged frontline providers and staff or gathered data about the impact of their initiative. Common challenges included a lack of 
leadership support, difficulty securing data, and competing organizational priorities. Framing the work around physical, emotional, or financial harm to patients was important in efforts to engage providers and staff. Those leading the projects emphasized that changing complex, entrenched practices required significantly more time and effort than anticipated.

Conclusion: Although overuse reduction shares similarities with the areas of traditional quality improvement and patient safety, this work requires unique approaches and more time and effort than often anticipated. Key elements of successful overuse-reduction efforts include engaged leadership and a focus on reducing patient harm. Future work is needed to explore which combinations of strategies are most effective in supporting overuse reduction.

\section{P3.01}

\section{Patient Perspectives and Patient-Reported Barriers to Kidney Care From Southeastern Chronic Kidney Disease Patients}

Jennifer Gander, ${ }^{1}$ Musu Sesay, ${ }^{1}$ Doraina Walker Williams, ${ }^{1}$ Lee Cromwell, ${ }^{1}$ Teaniese Davis, ${ }^{1}$ J. Patrick Austin, ${ }^{1}$ Adam Wilk, ${ }^{2}$ Rachel Patzer, ${ }^{2}$ Nirvan Mukerji ${ }^{1}$

${ }^{1}$ Kaiser Permanente Georgia, Atlanta, GA; ${ }^{2}$ Emory University, Atlanta, GA

Background: Treatment education significantly improves patient outcomes and access to care for the growing number of patients with advanced chronic kidney disease (CKD). Few studies have examined CKD patient-reported experiences regarding accessing kidney care, including key barriers and facilitators, and how their experiences may be augmented through patient education. We conducted a qualitative study to capture the experiences of advanced CKD patients accessing kidney failure treatment, including any modifying effects of CKD treatment education, within a southeastern integrated health care system.

Methods: We completed 3 focus groups with 2 types of patients with advanced CKD: a) 2 focus groups among patients referred and attended the kidney treatment education class (attendees); and b) 1 focus group of patients who were referred and did not attend the education class (nonattendees). Each participant gave written consent and was asked about 1) patient-health care provider relationships, 2) kidney care education, and 3) social support.

Results: Preliminary results from the 3 completed focus groups involved $17 \mathrm{CKD}$ patients $(76.7 \%$ black, $47.1 \%$ female) with average age of 59 years. The "attendees" $(\mathrm{n}=11)$ reported being satisfied with their health care team and were encouraged by the formal kidney treatment education class; reported barriers included needing more information on CKD treatment options and uncertainty about treatment cost. "Non-attendees" $(\mathrm{n}=6)$ primarily reported only interacting with their nephrologist, reported transportation and scheduling conflicts as barriers to attending the education class, and were interested in other modes of education. Both attendees and non-attendees reported having a strong social support system. Attendees and non-attendees reported feeling overwhelmed with their advanced CKD diagnosis and feeling that they had insufficient time between being referred for the class and when they would start dialysis.

Conclusion: Kidney treatment education was informative but could be improved by occurring more frequently and offering information on treatment cost. We plan to complete 1 additional focus group among non-attendees and 2 focus groups among patients that achieved a non-catheter-based dialysis start (optimal starters). In our efforts to close the information gap for patients with advanced CKD, we will continue to enhance our patient engagement processes utilizing the results from our focus groups.

\section{P3.02 \\ MedTrue $^{\mathrm{TM}}$ : Triangulating Patient-Reported Data With Electronic Health Record and Claims Data to Create Accurate Medication Lists and Facilitate Conversations}

Michael Gionfriddo, ${ }^{1}$ William R. Kaledas, Jr., ${ }^{1}$ Katie Frusciante, ${ }^{1}$ Melissa S. Kern, ${ }^{1}$ Nevan Elder, ${ }^{1}$ Stephen Castor, ${ }^{1}$ Eric Wright ${ }^{1}$

\section{${ }^{1}$ Geisinger Health, Danville, PA}

Background: Medication lists are often incomplete, which can lead to serious adverse consequences. To help patients and their clinicians have better conversations around medication use and to create a more accurate medication list for care management, we developed MedTrue ${ }^{\mathrm{TM}}$. MedTrue is a web-based, electronic health record (EHR)-agnostic, workflow application that integrates medication data sources related to reconciliation and adherence from the patient, provider, and external pharmacy claims systems, such as Surescripts $^{\circledR}$. Herein, we describe MedTrue and discuss the development process.

Methods: MedTrue was developed using an iterative design process that included clinical observations and feedback from end users. Project team members were embedded in the clinic test sites to identify and troubleshoot technical issues, gather observations on usability and utility, and collect enhancements for the application.

Results: MedTrue has both a patient-facing and a health care professional-facing interface. Initial prototypes of MedTrue covered a comprehensive review of medication use and contained several different screens addressing not only medication reconciliation but medication adherence and medication scheduling. Based on stakeholder feedback, adherence and reconciliation screens were combined and the scheduler screen was removed due to minimal utility with the current use case. MedTrue can be accessed by patients ahead of a scheduled office visit via our patient 
portal and immediately in the clinic prior to the office visit through an iPad. Once accessed, patients can confirm their medication list, add any additional medications, and report their adherence to their medications over the last month. Health care professionals can access MedTrue through the patient's EHR from within an embedded iFrame or a web link opening the application in a browser. Health care professionals can then review a patient's medication changes as well as existing medications, which are also listed with patient-reported and pharmacy claims adherence data. To date, MedTrue has been accessed by more than 7000 patient/ clinician dyads with over 35,000 unique uses.

Conclusion: MedTrue ${ }^{\mathrm{TM}}$ is a novel technology solution that aims to create better conversations around medication use and produce more accurate medication lists, ultimately improving patient safety and outcomes. We are currently evaluating MedTrue in a cluster-randomized trial.

\section{P3.03 \\ Developing a Center for Evaluation in a Learning Health System}

\author{
Meghan JaKa, ${ }^{1}$ Jeanette Y. Ziegenfuss, ${ }^{1}$ Jennifer \\ Renner, ${ }^{1}$ Casey Easterday, ${ }^{1}$ Nico Pronk ${ }^{1}$ \\ ${ }^{1}$ HealthPartners Institute, Minneapolis, MN
}

Background: Measuring program effectiveness is necessary in a learning health system. A suite of services typically exists to assess various aspects of program success (eg, quality improvement, health informatics). However, these specialized services are not intended to be cross-functional, nor do they have methodologic expertise needed for systematic program assessment. Research can address this gap, but reliance on external funding results in misaligned timelines and competing priorities. Evaluation services have the potential to complement research efforts by leading crossfunctional collaboration of existing services to rigorously assess programs on tighter timelines and potentially lower costs. The development of an evaluation center within a research setting is presented here. Specific challenges, including demonstrating value, establishing stakeholder buy-in, and developing operations, will be addressed.

Methods: To meet growing demand, a pilot evaluation service line was established within a learning health system's research institute. Through several test projects, the service line expanded its existing skillset and cultivated client "champions." In parallel, a development team with expertise in research, evaluation, business intelligence, and project management was established to formally develop the service line into a center. Informed by feedback from center members, leaders, and stakeholders, the team held a series of meetings to develop a strategic direction and establish goals for creating a solvent center.

Results: This process resulted in securing stakeholder buy-in through client champions, hiring new expertise, and developing a core set of plans focused on strategic direction, operations, collaboration, and center performance. Important performance goals such as number of repeat customers and right-sized budgets will be assessed over the coming year. An advisory board and peer-review process for evaluation products also were developed to ensure rigorous methodology and promote growth of the newly formed center. As evidence of early success, the center has secured operational funds to implement a number of programs in its first year and is regularly receiving new project requests.

Conclusion: Continued success of the quickly growing center depends on fostering strong relationships, prioritizing and operationalizing projects, leveraging scientific rigor, and receiving ongoing input from key stakeholders. Conceptual, operational, and fiscal lessons learned during the first year of implementation will be discussed.

\section{P3.04}

\section{Screening and Referral of Orthopedic Patients Into Care Coordination to Decrease Readmissions}

\author{
Danielle Miller, ${ }^{1}$ Theodore Manson, ${ }^{2}$ Nathan O'Hara, ${ }^{2}$ \\ Joan Davenport ${ }^{1}$
}

${ }^{1}$ University of Maryland School of Nursing, Baltimore, MD; ${ }^{2}$ University of Maryland Medical Center, Baltimore, MD

Background: Unplanned hospital readmissions negatively impact patient outcomes and continue to place a financial strain on the health care system. It is estimated that Medicare spends $\$ 17.4$ billion on unplanned readmissions in a year, and 30-day readmission rates are a hospital quality indicator reported by the Centers for Medicare \& Medicaid Services (CMS). Hospitals face monetary penalties for readmission rates that exceed national benchmark under the Affordable Care Act. In 2014, hip and knee replacements were added to the Hospital Readmission Reduction Program, thus authorizing CMS to financially penalize hospitals for readmissions within 30 days of discharge.

Methods: This is a quality improvement project aimed at reducing readmission in orthopedic patients undergoing a revision of a total hip replacement or total knee replacement. The LACE risk-screening tool identifies patients at high, intermediate, and low risk for readmission to refer those patients into care coordination. LACE index screening chronicled in multiple studies has proven to be a superior tool in determining readmission risks. The low-risk patients receive a telephone phone call prior to the first appointment postdischarge. The intermediate-risk and high-risk patients receive phone calls for 30 days postdischarge.

Results: Planned data analysis will examine the readmission rates in quarter one of 2018 (July-September) and compare those readmission rates in quarter two of 2018 (OctoberDecember) when the LACE screening tool was implemented. In addition, the number of no-show appointment rates will be compared from quarter one to quarter two of 2018 as 
well. Emergency department visits will be tracked in quarter two for patients screened by the LACE tool.

Conclusion: The LACE index is a screening tool to help health care systems make decisions on management of care based off likelihood of survival and readmission into the hospital. In 2010, Walraven et al endorsed this tool due to extensive testing in more than 1 million randomly selected medical and surgical patients after their discharge. Introducing care coordination can assist with adequate postdischarge support - increasing the likelihood of proper follow-up with the health care team - and improve hand-off between the inpatient and outpatient setting of health care.

\section{P3.05}

Direct-to-Patient Outreach to Improve Laboratory Monitoring of High-Risk Medications in Ambulatory Care

\section{Lisa Pieper, ${ }^{1}$ Susan Shetterly, ${ }^{1}$ Marsha Raebel ${ }^{1}$}

\section{${ }^{1}$ Kaiser Permanente Colorado, Denver, CO}

Background: Many high-risk medications require laboratory monitoring for safe use. At Kaiser Permanente Colorado (KPCO), we identified a quality gap in completing laboratory monitoring for high-risk medications, specifically disease-modifying antirheumatic drugs (DMARDs). Although most patients eventually completed monitoring, there was a gap in timeliness to monitoring. Herein, we describe the steps we took to develop and implement an intervention that successfully reduced the gap, present best practices for transitioning the work into routine operations, and discuss expansion to other departments and medications. Methods: KPCO is an integrated health care delivery system with about 600,000 members in the Denver/Boulder area. When organizational leaders were interviewed about quality improvement priorities, monitoring of high-risk medications was identified as of high importance. A multidisciplinary team comprised of laboratory leaders, content experts with knowledge of quality issues, clinical medicine and pharmacy experts, health information technology professionals, medical education specialists, evaluators, researchers, virtual data warehouse (VDW) experts, and project managers collaborated to develop/implement directto-patient interactive voice response (IVR) reminders. Reminders were sent by text or phone call to patients taking DMARDs who were due/overdue for monitoring. Data resources to ensure appropriate patients received correct reminders included the VDW, the electronic health record, and the IVR system. System outcomes were measured.

Results: Employing a multidisciplinary team to develop/ implement an intervention for a quality gap identified through an organizational leadership interview process enabled strong operational support for the project. IVR reminders to rheumatology patients were implemented by researchers in 2017 and transitioned into usual care in
2018. The reminders were supported by a patient helpline. Outcomes demonstrated 1) the proportion of patients chronically taking DMARDs, with guideline-concordant monitoring increased from $52.8 \%$ (baseline) to $65.4 \%$ (outreach) $(\mathrm{P}<0.001)$; and 2$)$ the 95 th percentile for time to monitoring declined from 149 days (baseline) to 117 days (outreach). Patient and clinician feedback has been positive. The project was expanded in December 2018 to additional high-risk medications prescribed by dermatology, gastroenterology, and neurology.

Conclusion: Automated communication outreach to remind patients to obtain ordered laboratory monitoring improves timeliness of testing for high-risk medications, is liked by patients, and has been incorporated into usual operations.

\section{P3.06 \\ Examining Workflow Issues When Designing a Clinical Decision Support Intervention to Reduce Opioid Prescribing for Dental Extractions}

\section{Brad Rindal, ${ }^{1}$ Robert P. Schwartz, ${ }^{2}$ Jan Gryczynski, ${ }^{2}$ Stephen Asche, ${ }^{1}$ Jeanette Y. Ziegenfuss, ${ }^{1}$ Don Worley, ${ }^{1}$ Anjali R. Truitt, ${ }^{1}$ Tracy Shea, ${ }^{1}$ Sheryl Kane, ${ }^{1}$ Shannon G. Mitchell ${ }^{2}$}

${ }^{1}$ HealthPartners Institute, Minneapolis, MN; ${ }^{2}$ Friends Research Institute, Inc., Baltimore, MD

Background: The implementation of clinical decision support (CDS) within an electronic health record (EHR) to reduce opioid prescribing following dental extractions poses significant challenges related to workflow and timely activation. Failure to consider these issues could reduce the effectiveness of the CDS intervention. We describe how these issues were addressed prior to developing a CDS for a trial focused on deimplementing opioid prescribing in dentistry.

Methods: Three study team members conducted observations of 15 dental extractions, with a focus on provider-patient interactions as well as provider use of the EHR as it relates to analgesic prescribing for extractions. A structured checklist was developed by the team and completed for each extraction observation, with follow-up clarifying questions asked of the clinical staff when necessary to better understand content accessed and sequencing of EHR-related activities. Activities were specified as occurring before or after the extraction.

Results: The observations provided valuable information about when provider-patient discussions of analgesic medications occurred and how the EHR was utilized during these interactions. The observations illuminated variation in processes and challenges of ensuring appropriate CDS activation in circumstances when a dental extraction is added into the treatment plan for a visit. Observations revealed that general dentists and oral surgeons have different workflow processes. These differences include 
when provider-patient discussion of analgesics occurs, the level of involvement of other clinic staff, EHR use, and visit types where dental extractions could occur. Findings have implications for informing CDS content as well as the content of implementation trainings.

Conclusion: CDS is a potential tool within an EHR that can be utilized to implement evidence-based practices or to deimplement ineffective and/or potentially harmful practices. These results foster our understanding of the issues and challenges of using CDS in dentistry.

\section{P3.07}

\section{System and Patient-Level Barriers to Timely Follow-Up Colonoscopy Completion: Qualitative Exploration Within Safety-Net Clinics}

Jennifer L. Schneider, ${ }^{1}$ Jennifer Rivelli, ${ }^{1}$ Inga Gruß, ${ }^{1}$ Amanda Petrik, ${ }^{1}$ Beverly B. Green, ${ }^{2}$ Gloria Coronado ${ }^{1}$

${ }^{1}$ Kaiser Permanente Center for Health Research, Portland, OR; ${ }^{2}$ Kaiser Permanente Washington Health Research Institute, Seattle, WA

Background: Fecal immunochemical testing (FIT) is an effective colorectal cancer (CRC) screening method. Its effectiveness, however, relies on follow-up diagnostic colonoscopy when the FIT result is abnormal. Many patients with an abnormal FIT result, particularly those within safetynet clinics, do not complete a timely follow-up colonoscopy. Methods: As part of the Strategies and Opportunities to STOP Colon Cancer in Priority Populations (STOP CRC) study, which tested the effectiveness of mailed FIT outreach to increase CRC screening rates in safety-net clinics, we conducted interviews with participating patients. We sought to understand facilitators and barriers to the timely completion of a follow-up colonoscopy after an abnormal FIT result. We interviewed both Spanish-language (SL) and English-language (EL) patients who completed their colonoscopy "early" ( $\leq 2$ months of FIT result), "later" ( $>2$ months of FIT result), or not at all. Interviews were completed by telephone, lasted approximately 45 minutes, and were transcribed for content analysis.

Results: We completed 32 patient interviews (16 SL, 16 EL), mostly with females (66\%), and representing 13 early completers, 14 later completers, and 5 noncompleters of follow-up colonoscopy. The most commonly stated challenge to timely colonoscopy completion was fear about the procedure, and this was endorsed more often by SL participants and those who completed later. SL participants cited as additional challenges cost and lack of clear information about the procedure, while EL participants described lack of reliable transportation. Being able to discuss concerns about the colonoscopy process with a provider prior to the procedure was the greatest facilitator to colonoscopy completion, endorsed strongly by both SL and EL early completers. Noncompleters of the colonoscopy (both SL and EL participants) described simultaneous barriers including not understanding their abnormal FIT result, having other health concerns, and cost.

Conclusion: Our interviews provide insight into challenges patients experience that can delay or prevent completion of a follow-up colonoscopy and add to our previous interview findings with gastroenterologists that showed lack of understanding, logistical issues, cost/insurance challenges, and poor referral documentation/communication created barriers to follow-up colonoscopy completion. Overall, these findings can inform the design of programs to improve rates of colonoscopy completion in safety-net clinics.

\section{P3.08 \\ Peer Support Needs of Young Adult Patients With Self-Identified Complex Medical Conditions: Opportunities for Improved Connection With Clinical Care}

\author{
Karen Wernli, ${ }^{1}$ Sarah Evers, ${ }^{1}$ Heidi Berthoud, ${ }^{1}$ \\ Consuelo Norris, ${ }^{1}$ Dori Rosenberg ${ }^{1}$
}

${ }^{1}$ Kaiser Permanente Washington Health Research Institute, Seattle, WA

Background: Younger adults with chronic or complicated medical conditions often experience different care needs. Peer support is a valuable tool to improve patient selfefficacy, knowledge, motivation, and patient empowerment. However, little is known about who is using peer support and why. We evaluated peer support needs among young adult members with chronic or complex medical conditions within Kaiser Permanente Washington (KPWA).

Methods: In the summer of 2018, we conducted via email a cross-sectional survey of KPWA members 25-45 years of age. Participants ( $\mathrm{N}=322 ; 3.8 \%$ of those contacted, $74 \%$ female, mean age: 37 ) self-identified as having a complex medical condition (receiving care for a condition that is not resolved by a single medical treatment, including surgeries). Statistical analysis was conducted in SPSS to evaluate descriptive statistics.

Results: Majority of respondents were currently receiving care for a chronic or complex condition $(78 \%)$ or had received care within the past 2 years (22\%). Most commonly reported conditions included mental health/depression/ addiction, inflammatory/autoimmune, women's health, musculoskeletal/bone disease, cardiac/vascular disease, and cancer; $25 \%$ of respondents used peer support for their condition. Among those who did use peer support, most common reasons for support were ongoing support for quality of life ( $\mathrm{n}=60,75 \%)$, and coping and to better understand or learn about their condition $(\mathrm{n}=61,76 \%)$. Among users of peer support, $25 \%$ were referred from their medical team. Most reported their medical care team was not aware of their peer support $(\mathrm{n}=49,61 \%)$. Of all survey respondents, almost $40 \%$ wanted their main health care provider, in particular 
their physician, to talk with them about getting peer support $(n=123)$. Further, respondents wanted to use online support groups (51\%), social media-based support group for people with the same conditions as them (36\%), in-person support group $(35 \%)$, or mentoring-based one-on-one peer support (34\%).

Conclusion: Patients with chronic and complex medical conditions reported self-needs for peer support across a variety of medical conditions. Young adult patients would like improved integration of their peer support needs with their medical team. Further research will be to develop interventions to support young adult patients through peer and online support tools.

\section{PATIENT, CLINICIAN, AND HEALTH SYSTEM ENGAGEMENT}

\section{OA4.01 \\ Characteristics of Learning Health System-Based Research Programs}

\section{Brian Mittman, ${ }^{1}$ Bhanuja Dub, ${ }^{1}$ Chunyi Hsu, ${ }^{1}$ Visanee Musigdilok, ${ }^{1}$ John Ovretveit, ${ }^{2}$ Michael K. Gould ${ }^{1}$}

\section{${ }^{1}$ Kaiser Permanente Southern California, Pasadena, CA; ${ }^{2}$ Karolinska Institutet, Stockholm, Sweden}

Background: Embedded or partnership research involving close collaboration between researchers and health system leaders is a promising strategy for enhancing the relevance, timeliness, value, and beneficial use of research in health system decision-making. Embedding researchers into health care systems and facilitating active, sustained collaboration and partnership between researchers and decision-makers can facilitate the identification of important research questions, improved study design and conduct, and more valid interpretation and application of findings. We sought to characterize existing embedded research programs in private and public academic and nonacademic U.S. health systems to identify features of successful programs.

Methods: Reviews of published descriptions of health system-based research programs, community-based participatory research, and stakeholder-engaged and partnership research, supplemented by discussions with key experts, guided development of a framework listing key features of such research and a new online survey collecting detailed descriptions of these features. Key domains in the framework and survey include organizational placement and governance arrangements (including details of policy/ practice and research leader collaboration in governance decisions), funding sources, staffing and inventory of skills and expertise, types of projects conducted, dissemination and implementation practices, etc. Respondents were identified through membership lists of relevant associations (eg, Health
Care Systems Research Network [HCSRN], Association of American Medical Colleges Research on Care Community, Veterans Affairs' Quality Enhancement Research Initiative [QUERI]), through a prior survey of health system-focused research conducted by AcademyHealth (Washington, DC), and through snowball sampling.

Results: Health system-based research programs are prevalent among academic health systems, large and mediumsized private health care systems, and private and public safety-net systems, in addition to well-established programs based at HCSRN member institutions and VA's QUERI. Programs vary widely in their organizational placement (eg, within an academic health system or associated medical school), funding (eg, support internal health system funding vs primarily on extramural research funding), staffing (fulltime, dedicated vs "virtual" staffing arrangements drawing from academic departments), and strength of connection to health system priorities and oversight.

Conclusion: Characterizing existing health system-based embedded research programs is a key step toward developing explicit guidance and recommendations for program features and toward growth in health system-focused research and learning health care systems.

\section{OA4.02}

OpenNotes in Chronic Obstructive Pulmonary Disease: Patient Perceptions of Their COPDSpecific Clinic Notes

Kimberly A. Fisher, ${ }^{1}$ Kara Kennedy, ${ }^{2}$ Sigall Bell, ${ }^{3}$ Mayuko Ito Fukunaga, ${ }^{2}$ Kathleen M. Mazor ${ }^{1}$

${ }^{1}$ Meyers Primary Care Institute, Worcester, MA; ${ }^{2}$ University of Massachusetts Medical School, Worcester, MA; ${ }^{3}$ Beth Israel Deaconess Medical Center, Boston, MA

Background: Patients report many benefits of access to their clinic notes (OpenNotes), including increased patient activation and self-management, which are particularly important in chronic illnesses such as chronic obstructive pulmonary disease (COPD). Despite this promise and increasing numbers of patients being provided access to their clinic notes, little is known about how patients understand and react to the information in clinic notes.

Methods: In-depth "think-aloud" interviews were conducted with patients with COPD who had been provided a written copy of their most recent COPD-specific clinic note. Interviews were audio-recorded, transcribed verbatim, and analyzed using thematic analysis. Emergent themes were organized according to communication function.

Results: Interviews were conducted with 31 participants who were predominantly (93.5\%) white, $48.3 \%$ female, and older (mean age: 65.9 years). Patients reacted positively to statements in the notes suggesting the provider listened to them ("He's got my thoughts in here, which ... makes me 
feel good about the fact that he listened to me."), saw them as a person, and was attentive to details ("I don't even realize that he does that."); all of which fostered the relationship. Reading notes enabled patient self-management through motivation ("seeing it [smoking history] in black and white makes you think"), prompted patients to seek more healthrelated information, and reminded them of action steps. Patients indicated notes facilitated information sharing with other providers and family members, served as a longitudinal record of care, and provided an opportunity for patients to learn details of their condition they had not gleaned from the visit ("I didn't get all this from the doctor. I got this from reading.") - each strengthening information exchange. Most patients reacted negatively to medical terminology, abbreviations, incorrect information, and wording that was perceived as disparaging ("He is an active smoker would've been fine, instead of putting abuse down there.").

Conclusion: Sharing clinic notes with patients is a promising communication tool that can strengthen the patient-provider relationship and enable self-management and information exchange. As patients increasingly access their clinic notes, more research is needed to ensure notes are written in a way that optimizes communication with patients while fulfilling other functions of clinical documentation.

\section{OA4.03 \\ Live Phone Calls More Effective Than Automated Reminders for a Direct-Mail Fecal Testing Program in Community Health Centers}

Jamie Thompson, ${ }^{1}$ Amanda Petrik, ${ }^{1}$ Michael Leo, ${ }^{1}$ Denis Nyongesa, ${ }^{1}$ Melinda Davis, ${ }^{2}$ Brittany Younger, ${ }^{3}$ Melissa Castillo, ${ }^{3}$ Anne Escaron, ${ }^{3}$ Gloria Coronado ${ }^{1}$

${ }^{1}$ Kaiser Permanente Center for Health Research, Portland, OR; ${ }^{2}$ Oregon Health \& Science University, Portland, OR; ${ }^{3}$ AltaMed Health Services Corporation, Los Angeles, CA

Background: Colon cancer is a leading cause of cancer death in the United States, and screening rates are disproportionately low among Latinos. Our study seeks to raise rates of colon cancer screening in a Latino-serving community health center. Using patient-refined materials, we examined fecal immunochemical test (FIT) completion rates following automated and live approaches in a 3-arm pilot pragmatic trial.

Methods: Eligible participants were 50-75 years of age, not up-to-date with colon cancer screening, and had a clinic visit in each of the previous 2 years at either of 2 health center clinics. A total of 1767 participants were randomized $(1: 1: 1)$ to receive a text alert, mailed FIT, and 2 automated phone call reminders (automated); a mailed FIT and up to 3 live call reminders (live); or the combination of text alert, mailed FIT, 2 automated call reminders, and up to 3 live call reminders (automated plus live). The format and content of the messages were selected using a patient-engagement approach, boot camp translation. We assessed FIT return rates in the 3 arms.

Results: Among randomized participants ( $\mathrm{N}=1767), 553$ (31.3\%) completed a FIT within 6 months. Participants had a mean age of 59 years and $57 \%$ were female. FIT completion rates were $26.0 \%$ (automated), $32.3 \%$ (live), and $35.7 \%$ (automated plus live). Controlling for clinic, adults allocated to the live condition had a significantly higher FIT completion rate than those in the automated condition (adjusted difference: 6.3 percentage points; 95\% CI: $1.1-$ 11.4), as did adults allocated to the automated plus live condition (adjusted difference: 9.7 percentage points; 95\% CI: 4.4-14.9). FIT completion rates differed significantly by preferred language (English: 25.5\%; Spanish: 35.1\%; Other: $39.7 \%$; $\mathrm{P}<0.01$ ); number of clinic visits in the past year (Yes: $33.9 \%$ vs No: $21.2 \% ; \mathrm{P}<0.01$ ); and prior FIT testing (Yes: $45.1 \%$ vs No: $17.1 \%$; $\mathrm{P}<0.01)$. No significant interactions were observed between treatment conditions and language, number of clinic visits, or prior FIT testing.

Conclusion: Live call reminders, either alone or with automated alerts and reminders, outperformed automated approaches alone. Using multiple touchpoints and communication modes might have the greatest impact on FIT completion rates.

\section{OA4.04 \\ Use of Novel Patient-Facing, Self-Directed Health Information Technology to Improve Patient Engagement in Preventive Care: Focus on Colorectal Cancer Screening}

Erin Hahn, ${ }^{1}$ Aileen Baecker, ${ }^{1}$ Eric Haupt, ${ }^{1}$ Andre Ajura, ${ }^{1}$ Wahid Wakach, ${ }^{1}$ Tracy Imley, ${ }^{1}$ Brian Mittman, ${ }^{1}$ Ernest Shen, ${ }^{1}$ Michael K. Gould, ${ }^{1}$ Michael Kanter ${ }^{1}$

\section{${ }^{1}$ Kaiser Permanente Southern California, Pasadena, CA}

Background: Engagement in health care via patient-facing online tools may be an effective method to increase use of preventive care services, including colorectal cancer (CRC) screening. Within Kaiser Permanente Southern California, the online patient portal includes an Online Personal Action Plan (oPAP), a tailored messaging tool intended to enhance patient engagement. We evaluated an oPAP CRC screening outreach strategy that allowed patients to directly request a CRC cancer screening kit (FIT Kit) to be delivered to their home using a self-directed "Order Kit" button.

Methods: Kaiser Permanente Southern California members, age 50-74 years, who were eligible for annual CRC screening and who had a portal account received the CRC oPAP "button" message from December 14, 2016, to October 2, 2017. Those who did not use the button were mailed a kit as a passive outreach strategy. We compared demographics, comorbidity, proxy measures of engagement 
(eg, missed appointments, portal use), and completion and timeliness of the FIT Kit for button-users vs non-users. We compared the number of button-users who completed a FIT Kit within the prior year to determine if the availability of the button engaged additional patients.

Results: We identified 177,209 eligible members; 9\% $(n=16,941)$ used the order button. Among button-users, $83 \%$ completed the FIT Kit vs $37 \%$ of non-button users $(\mathrm{P}<0.0001)$. Mean number of days to completion was 56 days in the button-users vs 90 days $(\mathrm{P}<0.0001)$. In multivariate regression, button-users were more likely to be white (odds ratio [OR]: $1.23, \mathrm{P}<0.001$ ), less likely to have 4 or more comorbidities (OR: $0.74, \mathrm{P}<0.001$ ), more likely to have 10 or more patient-portal logins in the past year (OR: 3.21, $\mathrm{P}<0.001$ ), and less likely to have no-show appointments (OR: 0.72, $\mathrm{P}<0.0001$ ). Of button-users, 12,076 were eligible for a FIT Kit in the prior year. Of those, 63\% $(n=7592)$ completed a kit, compared to $87 \%(n=10,448)$ post-button $(\mathrm{P}<0.0001)$.

Conclusion: A higher proportion of button-users completed FIT Kits than those who received the kit via the passive mail strategy; button-users completed the kits in a significantly shorter number of days. Of button-users, a significantly greater number completed FIT Kits after the button was introduced. Button use appears to reflect patient engagement, with greater number of health care contacts and fewer missed appointments for button-users.

P14.01

\section{Patient Self-Reported Symptoms Do Not Correspond With Asthma Exacerbations Identified in the Electronic Health Record}

Courtney Anderson, ${ }^{1}$ Glenn Goodrich, ${ }^{1}$ Susan Shetterly, ${ }^{1}$ Jo Ann Shoup, ${ }^{1}$ Nicole Wagner, ${ }^{1}$ Bruce Bender, ${ }^{2}$ Marsha Raebel ${ }^{1}$

${ }^{1}$ Kaiser Permanente Colorado, Aurora, CO; ${ }^{2}$ National Jewish Health, Denver, CO

Background: Filling a short-acting inhaled beta-agonist (BA) more frequently than recommended (overfill) is a predictor of asthma exacerbations. Asthma care nurses at Kaiser Permanente Colorado outreach to patients with BA overfills, a resource-intensive activity. To reduce this demand, the Breathewell project was implemented. Breathewell uses an automated system to contact patients overfilling a BA to identify patients currently experiencing asthma symptoms and those with recent exacerbations. However, whether patients' self-report of asthma symptoms is linked with asthma exacerbations is unknown. We therefore analyzed whether patients' self-reported symptoms agreed with asthma exacerbations identified within their electronic health record (EHR).

Methods: The intervention was conducted within Kaiser Permanente Colorado between February 2017 and February
2018. The study population included members, age 18 years or older, diagnosed with persistent asthma without chronic obstructive pulmonary disease. Members overfilling a BA received an automated (phone, text, or email) 1-question survey asking whether they experienced asthma symptoms in the last month. Asthma exacerbation data were collected from the EHR of patients responding to the survey for 1 month before and 1 month after the BA overfill. Asthma exacerbations were defined as prednisone bursts or emergent visits (emergency room, urgent care, or hospitalizations) with a primary diagnosis of asthma. Agreement was evaluated between survey responses and exacerbations using Cohen's kappa coefficient $(\kappa)$.

Results: Of the 1278 patients contacted by the automated system, 508 (39.7\%) responded to the survey. In the month prior to the BA overfill, of 11 patients with an exacerbation, 10 (90.9\%) reported symptoms; of 497 without an exacerbation, $293(59.0 \%)$ reported symptoms. Agreement between self-report of symptoms and past exacerbations was poor $(\kappa=0.023)$. In the month following the BA overfill, of 20 patients with an exacerbation, $16(80.0 \%)$ reported symptoms; of 488 without an exacerbation, $287(58.8 \%)$ reported symptoms. Agreement between symptoms and future exacerbations also was poor $(\kappa=0.027)$.

Conclusion: Poor agreement between symptoms and either past or future exacerbations indicates self-report of asthma symptoms does not correspond with asthma exacerbations.

\section{P14.02}

\section{Improving Clinical Research Through Citizen Scientist Engagement}

Janet Brishke, ${ }^{1}$ Christy Evans, ${ }^{1}$ Elizabeth Shenkman ${ }^{1}$

${ }^{1}$ University of Florida, Gainesville, $F L$

Background: Stakeholder engagement is an important part of the research process, yet many community stakeholders lack knowledge about clinical research. The OneFlorida Clinical Research Consortium utilizes community stakeholders (citizen scientists) to bridge the gap between researchers and community members by offering lay perspective on research studies. As not all citizen scientists are familiar with research logistics, this may sometimes foster miscommunication or unrealistic expectations between citizen scientists and researchers.

Methods: Using instructional design principles such as a formative evaluation, a multimedia approach, and learning assessments with color-coded feedback, an online, selfpaced curriculum was created to operationalize training of new citizen scientists. The curriculum was pilot tested with institutional review board approval from July 2017 to January 2018, and data was collected from initial scores on assessments completed at the end of each module as well as a postcurriculum evaluation.

Results: The overall score across all modules was $81 \%$, with 
scores ranging from $76 \%$ for the clinical and translational science module to $98 \%$ for the stakeholder engagement module. Notable differences were observed in the cultural competency module, in which younger participants scored higher than older participants $(90 \%$ vs $77 \%)$, and in the orientation to citizen science module, in which established citizen scientists scored higher than new members $(93 \%$ vs $81 \%$ ). Citizen scientists completed an evaluation that asked questions about comprehension, video quality, and assessment items. Most citizen scientists felt it was easy to understand the information in the video tutorials, while $75 \%$ of citizen scientists felt that the assessment items were comprehensive and $62.50 \%$ felt that the assessment items matched the learning objectives.

Conclusion: The citizen scientists felt this curriculum was an effective approach to providing background on topics relevant to their work. The instructional design principles utilized were rated favorably, with minor suggestions to strengthen the program for future implementation. Participants gained a deeper understanding of the processes involved in conducting clinical research and have been able to give more focused, actionable feedback. Understanding how research works can help community stakeholders better understand their contribution to research, offer critical feedback to help advance health care, and inform other community members of the value in participating in research studies.

\section{$\mathrm{P} 14.03$}

Clinician Engagement in Health Systems Research: A Survey of Clinicians Affiliated With a Clinical Data Research Network

Irina V. Haller, ${ }^{1}$ Pat Conway, ${ }^{1}$ Joseph A. Bianco, ${ }^{2}$ James Conniff, ${ }^{3}$ Steven Eyer, ${ }^{2}$ Rozalina McCoy, ${ }^{4}$ Sarah Olimb, ${ }^{1}$ Allise Taran, ${ }^{1}$ Tameka Sama,${ }^{5}$ Catherine A. McCarty 6

${ }^{1}$ Essentia Institute of Rural Health, Duluth, MN; ${ }^{2}$ Essentia Health, Ely, MN; ${ }^{3}$ Duluth Family Medicine, Duluth, MN; ${ }^{4}$ Mayo Clinic, Rochester, MN; ${ }^{5}$ Arizona State University, Phoenix, AZ; ${ }^{6}$ University of Minnesota, Duluth, $M N$

Background: Clinicians are critical stakeholders in patientcentered research, as they can make research relevant to real-world settings. However, engagement of clinicians in research can be challenging. Clinicians from two sites of the Patient-Centered Network of Learning Health Systems (LHSNet) were invited to participate in a survey regarding supports and barriers to engaging in research to better understand their experiences and inform strategies to increase engagement.

Methods: An anonymous electronic survey included multiple-choice and open-ended questions focusing on clinician experience and interest in research, barriers, and resources needed for engagement in research. Barriers and resources were analyzed using descriptive methods and their variation by clinician demographic characteristics, clinical role, and level of rurality of health care setting calculated. Scales regarding level of interest in conducting research, barriers, and resources needed for clinician engagement were created using factor analysis. Open-ended data were analyzed using NVivo and modified grounded-theory approach.

Results: Survey responses were received from 719 clinicians from community-based, academic, and integrated health systems (35\% response rate). Among respondents, $46.6 \%$ were physicians, $17.5 \%$ nurse practitioners, and $6.1 \%$ physician assistants. Most clinicians (75\%) reported no current engagement in research. The 13 items regarding research roles formed a reliable scale (Cronbach's alpha $=0.933$ ). Participant's score on the interest in research scale did not vary by gender, age, years in practice, or clinical role but did vary by level of rurality of health care setting $(\mathrm{P}<0.001)$. Lack of protected time for research and competing clinical responsibilities were the highestrated barriers, followed by access to other research team members, research time reimbursement at salary level, and organizational culture that values research activities. Ratings of barriers did not vary by age, level of rurality, or clinical role. Female respondents rated organizational barriers and lack of resources higher than males $(\mathrm{P}<0.05)$. When asked about the type of research they wish to engage in, participants identified a wide range of topics, including research methods, education, medical conditions, populations, organizational development, and care delivery models. Some reiterated their concern about participating in research because of the time involved.

Conclusion: Survey results provided a foundation for developing strategies for clinician engagement in health systems-based research.

\section{P14.04}

Creating Sustainable Infrastructure for Ongoing Patient Engagement: Qualitative Findings From the PORTAL Patient Engagement Council

Chalinya Ingphakorn, ${ }^{1}$ Carmit McMullen, ${ }^{1}$ Carolyn Taylor, ${ }^{1}$ Suzanne Gillespie, ${ }^{1}$ Charles Anderson, ${ }^{1}$ Mickie Bowe, ${ }^{1}$ Florence Kurttila, ${ }^{1}$ Loretta Nierat, ${ }^{1}$ Nicole Sanchez, ${ }^{1}$ Leslie Taylor, ${ }^{1}$ Janice Tufte, ${ }^{1}$ Henry Werch ${ }^{1}$

${ }^{1}$ Kaiser Permanente Center for Health Research, Portland, OR

Background: Nearly a decade ago, the Patient-Centered Outcomes Research Institute (PCORI) recognized that creating a sustainable infrastructure for meaningful and reciprocal patient engagement is essential to advancing medical care. PCORI established a network of clinical data research networks (CDRNs). One CDRN, PORTAL, represents Kaiser Permanente, HealthPartners, and Denver 
Health systems. PORTAL established a patient engagement council (PEC) to provide patient engagement resources aligned with PCORI's mission.

Methods: In 2014, PORTAL staff identified and recruited patient stakeholders to join the PEC. PEC members participated in training sessions on topics such as "Reading, Reviewing, and Critiquing Research Articles;" "Reviewing Grant Proposals;" "Using Twitter to Enhance Your Advocacy Voice;" and "Understanding Conference Posters." Each PEC member has completed a self-assessment of their advocacy skills and learning goals. They have attended national conferences to meet with researchers, learn about and comment on research findings, and further develop their expertise. PORTAL staff helped to create personal profiles and biosketches for PEC members that have been included in grant applications. There have been on average 10 members on the council, with some turnover.

Results: PEC members have responded to more than 50 requests from researchers. PEC members have made research more patient-centered by offering their input (mostly via webinar or email) about study conceptualization and design, proposal ideas, study implementation and data collection plans, manuscripts, and dissemination. Five of our PEC members are formal patient partners on grant-funded studies, and all PEC members now have enhanced their skills and confidence to make their advocacy voice heard across a broad range of research topics. With the conclusion of PORTAL funding, we offer a patient engagement toolkit as a freely available resource for researchers hoping to increase patient engagement.

Conclusion: The real-world experience and perspective that the PEC brings to research is needed and invaluable. We found that patients are eager to partner with researchers, to have their voices heard, and to develop the expertise to make meaningful contributions to research. They also have a message to researchers: "Seek us out!"

\section{P14.05}

Graphic Medicine as Physician Tool to Understand Their Patient's Experience of a Medical Condition

\section{Tom Janisse ${ }^{1}$}

\section{${ }^{1}$ The Permanente Federation, Portland, OR}

Background: Engaging patients in their health care, listening to their stories, and improving the quality of their experience also depend on physicians understanding their patient's experiences of a medical condition. Physicians have little time to converse with patients about this in the visit. Graphic medicine - the use of comics (pictures and words together in sequence to tell a story) - is a way to gain insight into a patient's experience of what it's like.

Methods: This is a small, mixed-methods study to test the effect on physicians of reading a comic book, "My Degeneration: Parkinson's Disease." The 13 participants, including 11 physician-editors (representing 10 disciplines), answered a 7-question presurvey before receiving and reading the book and a 10-question postsurvey. Also, the 12 participants present at the recent Permanente Journal editorial team meeting, held 3 days after the postsurvey, offered comments (60 minutes) on the survey results, then related their experience of reading the book, its attributes, and their recommendations for the comic book as an educational tool for residents and patients.

Results: Greatest improvements were "know patients' wants" (54\%), "know treatments" (37\%), "know patients' needs" (34\%), and "know patients' experience" (30\%); 82\% recommended the comic book for resident education and $73 \%$ for patients. Notable physician comments included: "My patients say: 'Doc, you guys really need to understand what's going on for me. It's really hard for me." Another senior editor who just disclosed he's been living with Parkinson's disease for 10 years exclaimed, "Excellent book! And I'm well into my 80s." Other comments were: "For a patient to have a conversation with his disease, as in the book, is a wonderful idea." "The things that people do to deal with their condition are remarkable!" "Combining pictures with words has triple the educational value for millennial residents who demand high yield." "Recommend for all who may be interested, including family members."

Conclusion: A graphic comic format improves the level of knowledge and treatment of a chronic medical condition, the understanding of a patient's needs and wants from a physician, and the patient experience, among a multidisciplinary group of physician-editors.

\section{P14.06 \\ Geisinger Provider Experience With Prior Authorization}

Ilene Ladd, ${ }^{1}$ Christina Gregor, ${ }^{1}$ Jove Graham, ${ }^{1}$ Michael Evans, ${ }^{1}$ Michael Gionfriddo, ${ }^{1}$ Laney Jones ${ }^{1}$

${ }^{1}$ Geisinger Health, Danville, PA

Background: The prior authorization (PA) process was created to improve appropriate use of prescription medications and to manage health care costs. However, the process as currently practiced has engendered high levels of dissatisfaction among medical staff. The American Medical Association (AMA) issued a survey in 2017 to assess physicians' views on the impact of PA on patients and providers. We adapted the AMA 2017 survey to establish a baseline snapshot of providers' views of PA prior to the implementation of an intervention to improve PA in our health system.

Methods: The 14-question survey was emailed to 2307 providers at Geisinger who had written any prescription since July 2018. A reminder email with a link to the survey was sent 1 week and 2 weeks after the initial survey. The first question of the survey screened out providers who had 
not written a prescription that required PA in the past year. Results: Overall, 509 providers responded to the survey for a response rate of $22 \%$. Of those who responded, 401 (79\%) reported they had prescribed a medication requiring PA. The majority of providers $(62 \%)$ reported waiting at least 1 day to receive PA approval; $88 \%$ of the respondents believe that the PA process delayed access to necessary care, and $89 \%$ said the PA process led to negative impact on their patients' clinical outcomes. The burden of the PA process on providers and staff was described as high or extremely high by $80 \%$ of the providers, and $73 \%$ believed the burden of the PA process has increased over the last 5 years.

Conclusion: Overall, the perceived burden of the PA process to providers and patients was mostly similar with the AMA's study, but fewer local providers reported that the burden of PA had changed in the past 5 years $(73 \%$ vs $86 \%$, respectively.) After additional responses have been collected, this survey will serve as a baseline for provider views of the process prior to implementing a PA intervention at our system.

\section{P14.07}

\section{Developing a Research Strategy in a Network of Outpatient Behavioral Health Clinics for Veterans and Their Families}

\section{Rajeev Ramchand, ${ }^{1}$ Anthony Hassan, ${ }^{1}$ Alice Kim, ${ }^{1}$ Crystal Shelton, ${ }^{1}$ Heather Chiarello ${ }^{1}$}

\section{${ }^{1}$ Cohen Veterans Network, Silver Spring, $M D$}

Background: The Cohen Veterans Network (CVN) was established in 2016 to provide low- or no-cost mental health care to veterans and their families. With 10 clinics open and 15 more scheduled to launch across the United States by 2020, CVN is currently establishing a research strategy to learn from its experiences and use this information to improve future performance.

Methods: Through consultation with the CVN scientific advisory board as well as a review of key research findings from existing learning health systems (ie, the Mental Health Research Network), a research strategy was developed specific to CVN's practices and operations.

Results: There are two elements to the CVN research strategy - the research foundation which establishes the processes that will guide CVN's research activities (eg, human subjects protection, quality assurance, and dissemination), and the research strategy itself. The strategy is composed of 5 interrelated areas of focus: the use of existing data; the collection of new data; informing and improving clinical practices; enhancing business efficiencies; and contributing to the scientific collective.

Conclusion: A formal approach to conducting research will help ensure that CVN clients continue to benefit from highquality outpatient behavioral health care. The goal is for the strategy to be dynamic, with continuous adaptations as CVN continues to learn how to incorporate research learnings into program improvement and contributes to the emerging science of health systems and mental health research.

\section{DATA SCIENCE, INFORMATICS AND DATA MODELS}

\section{OA5.01 \\ Variation in Care Improvement Opportunities Suggests Need for Tailored Clinician-Specific Improvement Interventions}

Patrick J. O'Connor, ${ }^{1}$ Jeffrey Anderson, ${ }^{1}$ JoAnn SperlHillen, ${ }^{1}$ A. Lauren Crain, ${ }^{1}$ Jay Desai, ${ }^{1}$ Pritika Kumar, ${ }^{1}$ Rebecca Rossom ${ }^{1}$

\section{${ }^{1}$ HealthPartners Institute, Bloomington, $M N$}

Background: Various organizations, including Centers for Medicare \& Medicaid Services, have defined accountability measures that are publicly reported and used to adjust payment for clinical services. We posit that if primary care clinician (PCC) performance on accountability measures (such as diabetes or cardiovascular care) is suboptimal, then PCC-specific care improvement opportunities can be identified through automated monitoring of a broad set of specific underlying process-of-care measures pertaining to clinical actions.

Methods: As part of a clinical decision support (CDS) system implemented within a large health care delivery system, we provided individualized patient treatment recommendations related to optimal management of 6 cardiovascular risk factors at adult encounters. We developed process-of-care measures related to recognition of conditions, timely monitoring of disease states, time to medication intensification for patients in suboptimal control, medication choices, frequency of drug/condition and drug interaction safety concerns, and appropriate referrals by calculating the provider's proportion of patients that each of 60 clinical recommendations were generated by the CDS system (10 blood pressure, 15 glucose, 29 lipid, 4 aspirin, and 2 smoking). We then quantified variation across 597 PCCs (minimum 20 patients) on these process-of-care measures by analyzing the data from 128,679 clinic visits made by 75,855 adults with diabetes or cardiovascular risk factors from January 1, 2017, through December 31, 2017. Providers were classified as high-performing (10th percentile) and low-performing (90th percentile) for each recommendation based on the proportion of patients for whom the recommendation was generated.

Results: We observed substantial variation when comparing low- vs high-performing providers, with as much as a $29 \%$ difference in the proportions of patients for whom CDS recommendations were generated. Across measures, low- 
performing providers had a proportion of patients for a CDS recommendation at least 1.9 times greater than highperforming providers.

Conclusion: In a care delivery system frequently recognized for high-quality care, we observed many clinician-level opportunities to improve underlying processes of care that are linked to optimal diabetes and cardiovascular risk factor management. Wide variations observed in performance on process of care presents actionable opportunity to design and deliver PCC-specific quality improvement interventions. The dissemination potential for this automated surveillance technology is high.

\section{OA5.02}

\section{Using Seasonal-Hybrid Extreme Studentized Deviate Method to Detect Anomalies in Virtual Data Warehouse Quality Assessment}

\author{
Qing Zhou, ${ }^{1}$ Matthew Slaughter, ${ }^{1}$ Ning Smith, ${ }^{1}$ Alan \\ Bauck $^{1}$
}

\section{${ }^{1}$ Kaiser Permanente Northwest, Portland, OR}

Background: Kaiser Permanente's Center for Effectiveness \& Safety Research (CESR) conducts data quality assessments of virtual data warehouse (VDW) data regularly to ensure that the data collected through the VDW meet the specification defined by the data dictionary. The goal of this quality check is to reveal any potential issues in the data and the extract, transform, load (ETL) process by detecting anomalies in the counts of the raw records at different time intervals.

Methods: In the past, the moving average (MA) method has been used successfully to detect regular outliers without seasonality trend but failed to differentiate the recurrent seasonal jumps/drops from the real outliers when seasonality exists (for example, number of flu vaccination encounters). To solve this problem, we used the seasonal-hybrid extreme studentized deviate (S-H-ESD) method, which builds upon the generalized extreme studentized deviate test, to detect the anomalies. In this project, we applied the method to monthly encounter counts of 5 encounter types within the VDW where known anomalies exist at different time points. An open-source R software package named AnomalyDetection was used to perform the analysis. We also conducted MA analysis for comparison purpose.

Results: The results showed that, using AnomalyDetection, the S-H-ESD method correctly identified all anomalies while avoiding the seasonalities. In contrast, MA incorrectly labeled seasonal jumps as outliers. We also implemented the $\mathrm{R}$ code block in the SAS/Interactive Matrix Language procedure, which will allow us to deploy the $\mathrm{R}$ package directly from SAS and automate the AnomalyDetection process in the future.

Conclusion: Applying the seasonal-hybrid extreme studentized deviate method to data quality assessments complements and enhances the ability to correctly identify potential problems within the data.

\section{OA5.03}

Patient Navigation Following an Abnormal FIT: Use of a Predictive Model for Precision Delivery

\section{Eric Johnson, ${ }^{1}$ Matthew Slaughter, ${ }^{1}$ Sacha Reich, ${ }^{1}$ Gloria Coronado, ${ }^{1}$ Amanda Petrik ${ }^{1}$}

\section{${ }^{1}$ Kaiser Permanente Northwest, Portland, OR}

Background: Colon cancer is a leading cause of cancer death in the United States. Only $\sim 70 \%$ of Kaiser Permanente Northwest patients who are due for a follow-up colonoscopy obtain one within 6 months of their positive fecal immunochemical test (FIT) result. Patient navigation can increase follow-up colonoscopy rates but is "high-touch," and not all patients need it. This study, Colorectal Cancer Screening Follow-up Risk at Kaiser Permanente Northwest (KPNW CRC Risk), is a pilot for testing patient navigation among patients determined to be unlikely to complete a follow-up colonoscopy.

Methods: Patients were 50-75 years of age, had an abnormal FIT test, and were due for a colonoscopy. The risk prediction model included data from the electronic health record. Patients are assigned a risk prediction model-generated "colonoscopy adherence score" based on their probability of completing colonoscopy. Patients are randomized, within probability strata, to a phone-based program of patient navigation or to usual care. Patient navigation was conducted through the delivery system and within the KPNW gastroenterology teams. Primary effectiveness outcomes are colonoscopy completion within 60 days and time to colonoscopy receipt.

Results: To date, 320 patients have been randomized (160 assigned to patient navigation, 160 to usual care). All patients have a predicted probability of less than 0.70 of obtaining a colonoscopy within 6 months. With 60 days of followup, across all probability groups, more patients receiving navigation support are completing their colonoscopies than usual care patients $(50.0 \%$ [95\% CI: 36.9-63.1] vs $40.8 \%$ [95\% CI: 28.2-54.8]). The number of days from abnormal FIT result to colonoscopy completion is less in the patient navigation group than in the usual care group. On average across all probabilities at 60 days, the colonoscopies are completed about a week earlier (6.8 days). In the 50\%-60\% probability range, colonoscopies are completed about 3 weeks earlier with patient navigation than in the usual care group.

Conclusion: Knowing which patients could benefit from patient navigation could optimize the delivery of these services, address health disparities, reduce associated costs, and support broad adoption of patient navigation programs. Further application of risk prediction modeling for health services delivery should be explored. 
OA5.04

DIY Analytics for HMOs: How You Can and Why You Should

Roy Pardee, ${ }^{1}$ David Cronkite ${ }^{1}$

${ }^{1}$ Kaiser Permanente Washington Health Research Institute, Seattle, WA

Background: There is a robust market for predictive analytic products in both health care and health insurance. Some provide locally installed software (eg, $\mathrm{ACG}^{\circledR}$ System at Johns Hopkins University [Baltimore, MD]), while others offer services that give predictions for uploaded data (eg, Jvion [Johns Creek, GA]). Both are expensive but provide significant value. The increased availability and quality of open-source software for data science re-opens the "build or buy" question. Many of our organizations straddle both market segments (providers and insurers), affording more data than is used by most vended software. We hypothesize that this combination of data may yield greater insights than either would alone. We will tell the story of an effort at "building" a local model - similar to the risk of hospitalization component in ACG - to predict who is likely headed into the hospital for prioritizing case management efforts.

Methods: We pulled diagnoses, procedures, pharmacy fills, body mass index measures, blood pressures, and lab results for a cohort of 70,000 chronically ill patients observed during a 3-month period in 2016. We then identified inpatient admissions among those patients in the subsequent 12 months. Using a data science approach, we trained several classifiers from Python's scikit-learn package to discriminate between patients who were and were not hospitalized on the basis of their claims and clinical data together. We used the remainder of the data to evaluate those trained classifiers.

Results: The support vector (SVC) and random forest (RF) classifiers performed best, producing samples of more than $50 \%$ hospitalized people among their top 100 most-likely patients, comparable to the predictor of inpatient risk that ACG produces (SVC: 63\%; RF:55\%; ACG:46\%), though ACG relies on 12 months of data prior to the observation period rather than our use of only 3 months.

Conclusion: With modest efforts, virtual data warehouse data, free software, commodity hardware, and almost no clinical expertise, we constructed a predictor of inpatient risk that worked as well for our purposes as ACG, despite using fewer months of input data. Our results demonstrate that "building" analytics tools is a viable choice for organizations desiring to explore analytics, especially if they have access to more data sources than is expected by vended solutions.
P6.01

\section{An Integrated Analytic Database to Support Genomic Research}

\author{
Alan Bauck, ${ }^{1}$ Gwyn Saylor, ${ }^{1}$ Anand Sethuraman ${ }^{2}$
}

${ }^{1}$ Kaiser Permanente Center for Health Research, Portland, OR; ${ }^{2}$ Kaiser Permanente Program Office, Oakland, CA

Background: The Kaiser Permanente Research Bank (KPRB) is one of the most population-diverse biobanks in the United States and contains collections of blood, saliva, and diverse patient data for more than 330,000 individuals. Eight geographically separate KP regions participate in the KPRB. Participants consent to broad use of their biospecimens, electronic health record data, survey data, and genomic data for health research. To make its data available for precision medicine and genomic research, the KPRB developed a single integrated analytic database. Nine research investigators with knowledge of a broad range of research domains developed a comprehensive list of electronic health record (EHR) data to include in the database. The Center for Effectiveness and Safety Research (CESR) virtual data warehouse (VDW) was identified as the primary source for EHR data.

Methods: Data extraction programs were developed to pull data from the 8 participating regions' CESR VDW for the KPRB-consented participants, perform quality checks, and load the data into the KPRB analytic database. In addition to incorporating a large portion of the CESR VDW, the KPRB analytic database also includes a thousand-item survey, demographic information, and lab specimen data on KPRB-consented participants. To facilitate researchers' understanding of the KPRB analytic data, a population summary report was developed that can be regenerated using an existing data characterization program. The report provides more than 70 pages of summary statistics about the KPRB population and can be readily enhanced.

Results: The KPRB analytic database resides in a Microsoft SQL Server database with schemas for EHR, survey, and subjects and includes a total of 58 tables. All the data are linked through 1 of 3 key linking identifiers or child linking identifiers. The linking identifiers are accessible only within the database and not shared. When data are provisioned for researchers, a project-specific linking identifier will be generated, enabling access for that project. A comprehensive data dictionary of the database is built and maintained.

Conclusion: The integrated KPRB analytic database incorporates and links EHR, survey, demographic, and specimen information. It provides a rich and curated set of data from which KPRB analysts can efficiently provision data needed for health research. 
P6.02

The Center for Effectiveness and Safety Research Virtual Data Warehouse (VDW) Model, an Extension of the Health Care Systems Research Network VDW

Alan Bauck, ${ }^{1}$ Don Bachman, ${ }^{1}$ Phillip Crawford, ${ }^{1}$ Reesa Laws, ${ }^{1}$ Tamara Lischka, ${ }^{1}$ Gwyn Saylor, ${ }^{1}$ Sanchita Sengupta, ${ }^{1}$ Jenny Staab ${ }^{1}$

\section{${ }^{1}$ Kaiser Permanente Northwest, Portland, OR}

Background: The virtual data warehouse (VDW) for Kaiser Permanente's Center for Effectiveness and Safety Research (CESR) is a distributed set of standardized research data tables. In addition, it is a set of common data warehouses that are maintained by each site. The CESR VDW extends and complements the core patient/health care data in the Health Care Systems Research Network (HCSRN) VDW to include new data domains, such as patient-reported outcomes, radiation therapy, personal health records, pregnancy, benefits, bone mineral density, infusion, and additional medication data. Methods: CESR collects input on potential data domains to standardize for multisite research through surveys, meetings, and informal feedback. That information is collated to identify areas of high interest, and those with the highest interest are then explored through data feasibility assessments. The data feasibility assessments look at data availability, likelihood of use, and effort to develop and maintain. Data domains that pass the feasibility assessment are developed through multisite workgroups of researchers and programmers. The final deliverables are 1) a standardized and well-specified data dictionary; 2) common ETL (extract, transform, load) code, where applicable, or agreed-on business rules to transform the data to the new standard; 3) data that meet the common data specification and are refreshed and available for use by all researchers at all sites; and 4) a data characterization report summarizing the new data across the sites.

Results: The CESR VDW expands on the HCSRN VDW and more than doubles the number of tables available for multisite research from 19 to 52 tables and growing. The data definitions and specifications are readily shared with organizations seeking to build all or parts of the data for collaboration.

Conclusion: The CESR VDW complements and enhances the HCSRN VDW. It provides researchers the ability to efficiently conduct health research across organizations with an expanded set of research domains.

\section{P6.03}

\section{An Algorithm to Identify Ongoing Pregnancies in the Vaccine Safety Datalink}

Brad Crane, ${ }^{1}$ Don Bachman, ${ }^{1}$ Stephanie Irving, ${ }^{1}$ Kimberly Vesco, ${ }^{1}$ Eric Weintraub, ${ }^{2}$ Natalie McCarthy, ${ }^{2}$ Allison Naleway ${ }^{1}$
${ }^{1}$ Kaiser Permanente Center for Health Research, Portland, OR; 'Immunization Safety Office, Centers for Disease Control and Prevention, Atlanta, GA

Background: Using data from 7 integrated health care systems (sites), the Vaccine Safety Datalink (VSD) monitors vaccine safety. Evaluating vaccine safety during pregnancy is a priority for the VSD. The existing VSD Pregnancy Episode Algorithm (PEA) is effective at identifying pregnancy episodes after a pregnancy outcome but is unable to identify ongoing pregnancies. To address this limitation, we developed the Dynamic Pregnancy Algorithm (DPA) to identify pregnancies with or without pregnancy outcomes in the electronic medical record or claims data.

Methods: The DPA identifies medical encounters associated with pregnancies (pregnancy indicators) and incorporates coded diagnostic, procedural, and gestational age data to estimate several pregnancy-related variables. To test functionality, we implemented the DPA at all VSD sites to identify pregnancies occurring during 2016. Using the VSD PEA as a gold standard, we retrospectively compared DPA results with the PEA to 1) assess how quickly the DPA was able to identify pregnancy episodes, and 2) assess data quality of pregnancy episodes identified exclusively by the DPA. Results were stratified by live birth and non-live birth outcomes.

Results: The DPA identified 243,409 pregnancy episodes in 2016. Of those, 152,779 (63\%) had outcomes in 2016 identified by the PEA. Across sites, 99\% of PEA episodes with outcomes in 2016 were found by the DPA (range: 98.4\%-99.8\%). The PEA identified 8971 live birth and 4,194 non-live birth outcomes that occurred in December 2016. Of these, the DPA found at least 1 pregnancy indicator 6 or more months prior to outcome for $89 \%$ of live births (range: $85 \%-92 \%$ ) and found at least 1 pregnancy indicator 1 or more months prior to outcome for $47 \%$ of non-live births (range: $45 \%-60 \%$ ). Of pregnancies identified by the DPA during February $2016(\mathrm{n}=86,147), 92 \%$ had subsequent outcomes identified by the PEA in 2016 (range: $86 \%-92 \%$ ). Among those DPA-identified pregnancies with no subsequent PEA outcome, $70 \%$ did not have continuous health plan enrollment for all of 2016.

Conclusion: The VSD DPA reliably captures pregnancy episodes relative to the VSD PEA. By not requiring outcomes to identify pregnancies, the DPA can identify, in real time, ongoing pregnancies previously not available for study in the VSD.

P6.04

Using Predictive Analytics to Decrease Appointment No-Shows

Sharon Fuller, ${ }^{1}$ Owen Kanaga, ${ }^{1}$ Sarah Wray, ${ }^{1}$ Yates Coley, ${ }^{1}$ Adam Elder, ${ }^{1}$ Emily Westbrook ${ }^{1}$

${ }^{1}$ Kaiser Permanente Washington Health Research Institute, Seattle, WA 
Background: Patients do not show up for $6 \%$ of their appointments at Kaiser Permanente Washington clinics, and another $5 \%$ of appointments are same-day cancellations. This costs millions of dollars each year in unused provider and resource capacity and makes it harder to achieve patient access goals. Patients' health is also at risk when they do not follow through on their care. In 2018, we implemented a predictive model from Epic, our electronic health record, to identify appointments with a high risk the patient will noshow. As part of Kaiser Permanente Washington's Learning Health System (LHS) Program, researchers are collaborating with the delivery system to evaluate the model's accuracy and implement improvements.

Methods: The Epic model estimates the likelihood a patient will no-show for a given appointment. The first prediction occurs when the appointment is made, followed by 1 prediction a day for the 7 days before the appointment. We examined the area under the curve (AUC) to assess the model's accuracy. The AUC for the current model is approximately 0.70 , which is respectable, but leaves room for improvement. We have developed an external dataset including the 22 stock Epic data elements, along with additional Epic variables of potential interest. To test improvements, we will first create our own prediction model using these variables and compare it with Epic's model. We will add or remove variables to attempt to increase the AUC. Our revised model can be built back into our Epic instance.

Results: We will present AUC results for our custom model, discuss relevant variables, and preview new interventions to decrease the no-show rate.

Conclusion: We plan to use our increased predictive capabilities to focus interventions where they will be most effective. Reducing no-shows will increase efficiency and improve patient access and care. The advanced data and biostatistical and analytic capabilities of the LHS Program have helped the enterprise to move quickly toward an important strategic and operational goal. This collaborative effort also provides the opportunity to begin developing a framework for harnessing the power of predictive analytics for future projects.

\section{P6.05 \\ Development and Validation of an Algorithm to Identify Chronic Obstructive Pulmonary Disease in Individuals Eligible for Lung Cancer Screening}

\section{Duc Ha, ${ }^{1}$ Chan Zeng, ${ }^{1}$ Andrea Burnett-Hartman, ${ }^{1}$ Brian Hixon, ${ }^{1}$ Caroline Joyce, ${ }^{1}$ Debra Ritzwoller ${ }^{1}$ \\ ${ }^{1}$ Institute for Health Research, Kaiser Permanente Colorado, Aurora, $\mathrm{CO}$}

Background: The balance of benefits and harms of lung cancer screening (LCS) can be optimized through better characterization of underlying comorbidities. Chronic obstructive pulmonary disease (COPD) is a major comorbidity that can modify outcomes in individuals eligible for LCS. This study's objective is to exploit uniquely available clinical and administrative data to develop and validate an algorithm to more accurately identify COPD in individuals eligible for LCS.

Methods: In a retrospective cohort study we identified individuals 55-80 years of age who are eligible for or have received LCS at a large integrated health care system from 2014 to 2017 . In addition to descriptive statistics, we develop multivariable logistic regression models to predict COPD using input associated variables: demographics (eg, age, sex, race, body mass index, tobacco exposure); diagnoses of COPD, asthma, and other comorbidities captured within 2 years of the date of LCS eligibility or initial low-dose computed tomography by the respective providers (primary/pulmonology) and clinical context (outpatient/inpatient); pharmacy dispenses related to COPD and smoking cessation; durable medical equipment claims (eg, supplemental oxygen, nebulizers) and spirometry data (eg, peak expiratory flow rate, FEV1, forced vital capacity); health care utilization related to COPD exacerbations (eg, emergency department visit, hospitalization); and referrals to smoking cessation and pulmonary rehabilitation programs. We will use the Global Initiative for COPD definition as the reference standard and consider alternative diagnoses of obstructive airway diseases (eg, chronic obstructive asthma, bronchiectasis). The model performance will be evaluated by discrimination, calibration, sensitivity, and specificity.

Results: We identified more than 16,000 individuals who were eligible for LCS, of whom 3375 received baseline lowdose computed tomography. The median age was 64 years; most $(56 \%)$ were male, current smokers $(>55 \%)$, and had $\geq 30$ pack-years of tobacco exposure $(95 \%)$. The majority $(>50 \%)$ of eligible or screen patients had a diagnosis of asthma, COPD, or both, and received 1 or more COPDrelated pharmacy dispenses. Efforts are underway to capture spirometry values and develop multivariable logistic regression models.

Conclusion: Using a unique set of clinically derived input variables from a cohort of patients potentially exposed to both the benefits and harms of LCS, our algorithm may enhance the effectiveness, precision, and delivery of populationbased LCS by more accurately identifying eligible patients with COPD.

\section{P6.06}

\section{Validating the Use of Order Instructions to Predict Days Supply}

Luesa Jordan, ${ }^{1}$ Dustin Key, ${ }^{1}$ Lawrence Madziwa, ${ }^{1}$ Matthew Nguyen ${ }^{1}$

${ }^{1}$ Kaiser Permanente Washington Health Research Institute, Seattle, WA 
Background: Pharmacy (Rx) dispensing data is a gold standard in measuring medication use in observational studies. Research studies using pharmacy data often require days supply. Days supply can be an important element in a study. For example, it may be required to determine the eligibility of a participant or exposure status at a given point. When days supply is not available, the pharmacy instruction can be used to impute it.

Methods: Days supply is equivalent to the ratio of drug quantity to pills-per-day (PPD) and can be estimated by the instruction field also known as SIG. The SIG variable provides directions for medication use (eg, "TAKE 1 TABLET BEFORE BED"). Most SIG values can be translated to PPD. To automate this process, the authors have built a macro that takes SIG values and pharmacy amounts to derive a PPD value. This macro, which uses regular expression analysis, looks for common patterns of interest within each SIG value ("X TIMES A DAY," "EVERY X HOURS," etc). We compared the algorithmderived days supply to that of a pharmacy fills dataset for which the quantity was readily available. The mean absolute percentage error (MAPE) statistic and agreement accuracy were used for validation. Further stratification was done by drug form and SIG translation difficulty.

Results: After correcting for outliers, the PPD macro performs well against the validation data (MAPE mean: 17.1; overall accuracy: 76.2\%). For certain types of pharmacy dosage forms - tablets, capsules, and pills (TCP) - the macro performs even better $(8.1 ; 85.3 \%)$. For all other dosage forms, the macro performs poorly $(81.4 ; 11.9 \%)$. Within TCP, performance on SIGs that were classified as difficult is moderately poor $(64.2 \% ; 19.0 \%)$.

Conclusion: The PPD program can be a valuable tool for studies needing to analyze a prescription's days supply. Careful application of the program is necessary, however, due to known limitations/potential pitfalls (eg, weaknesses in handling certain dosage forms, inability to consider tapering, receiving SIG values with protected health information from outside sites).

\section{P6.07}

\section{Visualizing the Benefits Characteristics of a Large Health Care Provider's Membership}

\section{Don McCarthy, ${ }^{1}$ Mike Santema ${ }^{1}$}

\section{${ }^{1}$ Kaiser Permanente Southern California, Pasadena, CA}

Background: Visualization can be a valuable tool when dealing with complex data such as the benefits information associated with the membership of a large health care provider. We present several geographic visualizations of Kaiser Permanente Southern California (KPSC) membership insurance and benefits information.

Methods: We use the newly implemented at KPSC Center for Effectiveness and Safety Research benefits and the Health
Care Systems Research Network virtual data warehouse enrollment and census files (based on membership and benefit data extracted from KPSC membership data warehouse) as well as Base SAS software to create geographic visualizations of selected data elements (eg, copay, Medicare, Medicaid, Affordable Care Act status).

Results: We generate geographic visualizations of, for example, utilization and drug copay (in-network/out-ofnetwork), plan type, Medicaid status, Medicare status, and ACA status.

Conclusion: Mapping insurance data displays interesting and sometimes surprising patterns about the geographic location of different types of KPSC members and provides an excellent example of the power of geographic visualization.

\section{P6.08}

The Validation of a Predicted-Risk Model to Improve Completion of Colorectal Cancer Screening in Community Health Centers

Amanda Petrik, ${ }^{1}$ Eric Johnson, ${ }^{1}$ Matthew Slaughter, ${ }^{1}$ Carrie Nielson, ${ }^{1}$ Jamie Thompson, ${ }^{1}$ Ricardo Jimenez, ${ }^{1}$ Gloria Coronado ${ }^{1}$

${ }^{1}$ Kaiser Permanente Center for Health Research, Portland, OR

Background: Colorectal cancer (CRC) screening by annual fecal immunochemical test (FIT) depends on follow-up colonoscopy after an abnormal FIT to remove precancerous lesions or expedite CRC diagnosis and treatment. Up to $50 \%$ of FIT-positive patients fail to complete this essential followup component. Predicting and Addressing Colonoscopy in Safety Net Settings (PRECISE) aims to validate and apply a risk-prediction model to identify patients who are likely to forgo follow-up colonoscopy.

Methods: The original risk-prediction model from a prior study identified patients, 50-75 years of age, with an abnormal FIT result. Electronic health record data were used in a Cox regression model to predict colonoscopy completion within 6 months of an abnormal FIT. In the PRECISE project, we assembled a similar cohort and validated the original riskprediction model.

Results: The original model included 5-year age group, nonwhite race, insurance type, quintiles of income inequality (by Gini index), long-term anticoagulant use, receipt of a flu vaccine in the past year, frequency of missed clinic appointments, and clinic site. The PRECISE validation model produced a naïve c-statistic, and because of the high slope shrinkage and inapplicability to the clinic setting, the model was redeveloped. The use of "health system" as a predictor was not applicable to this single health system, therefore we used county as a determinant of geographical differences, which remained a strong predictor. This, as well as a higher probability of colonoscopy, warranted redevelopment. We are refining the model by updating the 
predictions using the baseline rate of colonoscopy at Sea Mar Community Health Centers to improve agreement between observed and predicted probabilities of colonoscopy and recalibrating the model's predictor coefficients by fitting a new Cox regression within the Sea Mar cohort.

Conclusion: The redeveloped model leverages readily available data in the electronic health record and tailors the model to the Sea Mar population. The model helps remove patients who would be unlikely to need patient navigation because of their high probability of follow-up. Predicting follow-up colonoscopy after an abnormal FIT allows clinics to target patient-navigation resources to patients who need it the most. The approach may represent a sustainable way to improve follow-up colonoscopy completion in community health center patients.

\section{P6.09}

\section{Tracking eConsult Note Views}

\section{Arvind Ramaprasan, ${ }^{1}$ James Ralston ${ }^{1}$}

${ }^{1}$ Kaiser Permanente Washington Health Research Institute, Seattle, $W A$

Background: Electronic consultations or eConsults are asynchronous, non-face-to-face consultations between the primary care provider (PCP) and the specialist via a secure web-based platform. In August 2017, Kaiser Permanente Washington providers were the first in the United States to invite patients to read the eConsult notes, which use the electronic health record (EHR) to connect a primary care provider (PCP) to a specialty provider for advice on a patient. As with all current clinic notes, eConsult notes are shared with the patients. The objective of this poster is to provide insight on patient viewership of eConsults and compare it with progress note views from face-to-face visits. Methods: We extracted face-to-face visits and eConsults from EHR and tracked the corresponding note views from January 1, 2017 to August 31, 2018. We used Tableau for visualization of results.

Results: During the study period there were 2,826,420 encounters, including 2,805,798 (97.5\%) face-to-face visits and 20,622 (0.7\%) eConsult referrals with associated progress notes accessible to 477,186 patients, and 70,507 $(2.5 \%)$ encounters either with progress notes hidden from patient view or without any notes attached. Of those accessible, progress notes from 187,499 (6.8\%) face-to-face visits and 1193 (6.4\%) eConsults were viewed at least once during the study period.

Conclusion: eConsults aim to simplify the process of seeking input from specialists, thereby reducing the need for specialty referrals that directly result in time and cost efficiencies. However, relatively few patients are viewing eConsult notes. We hypothesize that many patients are not aware that the eConsult notes are being shared and that those looking for notes may be experiencing difficulties finding them on the web portal. More research is needed to ascertain the reasons for low patient viewership of eConsults.

P6.10

\section{Converting Opioids to Morphine Milligram Equivalents for Research Purposes: Comparing an In-House Macro to Centers of Disease Control and Prevention's SAS Program}

\section{Arvind Ramaprasan, ${ }^{1}$ Ladia Albertson-Junkans ${ }^{1}$ \\ ${ }^{1}$ Kaiser Permanente Washington Health Research Institute, Seattle, $W A$}

Background: Over the years, health care organizations have independently developed algorithms to compute morphine milligram equivalent (MME) for standardizing opioid exposure for research and clinical purposes; however, inconsistencies in strength and conversion factors coupled with a lack of standardized programming approaches leaves room for error and compromises replicability and comparability across studies and data sources. The Centers for Disease Control and Prevention (CDC) developed and published a SAS program that calculates average daily dose (in MME) at the dispensing level. The SAS program is parsimonious and thus amenable to a variety of data models. It does not, however, include National Drug Codes (NDCs) for medications that are not typically used in outpatient settings, such as powders and solutions. To support current studies related to opioids, programmers at Kaiser Permanente Washington Health Research Institute (KPWHRI) recently developed a SAS macro for calculating average daily dose (in MME) at the dispensing level, using a comprehensive list of extended-release/longacting (ERLA) and immediate-release/short-acting (IRSA) opioids compiled and vetted by pharmacy experts across several health care sites. This poster examines two existing tools for calculating MME that researchers could use to ensure greater consistency, reliability, and programming efficiency in opioid-related studies.

Methods: We extracted 1,168,130 ERLA and IRSA opioid dispenses for 36,746 individuals from January 1, 2006, to September 30, 2015. We independently computed the daily MME per dispensing using the CDC and KPWHRI SAS tools. We used Tableau to visualize results.

Results: Comparing results from KPWHRI and CDC tools show $99.967 \%(n=1,167,740)$ concordance in daily MME. There were $342(0.03 \%)$ dispensing records with discordant daily MME, of which 53 (13.6\%) dispenses, corresponding to 9 NDCs, had varying strengths and 289 (84.5\%) dispenses, corresponding to $3 \mathrm{NDCs}$, had different conversion factors in the two macros. Overall, neither of the two programs computed daily MME for 48 (0.004\%) dispensing records.

Conclusion: KPWHRI has developed an algorithm within its virtual data warehouse common data model for accurate computation of average daily dose in opioid 
MME. Preliminary results have shown a very high level of concordance between the KPWHRI macro and CDC program. Further validation on a larger sample and broader time frame could enhance robustness of the KPWHRI macro, potentially benefitting other opioid-related multisite studies.

\section{P6.11}

\section{Making the Kaiser Permanente Northwest Virtual Data Warehouse Compatible With SAS's FedSQL and DS2}

Matthew Slaughter, ${ }^{1}$ Catherine Cleveland ${ }^{1}$

${ }^{1}$ Kaiser Permanente Center for Health Research, Portland, OR

Background: The Health Care Systems Research Network (HCSRN) virtual data warehouse (VDW) facilitates research data collection and analysis across sites by providing a common set of tables according to standard definitions and formats. The distribution of SAS code across sites depends on the use of standardized table names provided as SAS macro variables and site-specific SAS data connections. New SAS features, such as the FedSQL and DS2 procedures, can improve VDW multisite programs by taking advantage of the SQL:1999 American National Standards Institute standard and allowing multithreaded data processing. However, changes to the Kaiser Permanente Northwest (KPNW) StdVars program were necessary for compatibility with these procedures.

Methods: The KPNW StdVars program must allow access to VDW data tables through a single set of data connections and macro variables, regardless of programming context. The FedSQL and DS2 procedures do not allow duplicate library connections to the same data source. Therefore, duplicate legacy library connections were eliminated from the program. In addition, current SAS releases do not support database management system schemas in FedSQL and DS2 data connections for some data sources. Consequently, standard 2-level SAS data references were replaced with 3-level table references, including the schema name when those tables were referenced in FedSQL or DS2. Initially, a separate StdVars program was created for these procedures, but this solution was insufficient, as 3-level names are not allowed in other contexts. To create a single StdVars program for use with all SAS procedures, the \&SYSPROCNAME automatic macro variable was used to make variables referencing VDW tables resolve to a 2- or 3-level name dynamically.

Results: Changes to the KPNW StdVars program allowed new SAS procedures to access KPNW VDW data using single set of data library connections and macro variables.

Conclusion: Issues with FedSQL and DS2 implementation will vary across HCSRN sites, but the KPNW experience may be a useful model. Full VDW compatibility with new SAS procedures will allow the use of potentially more efficient and powerful programming techniques in distributed SAS code.

\section{P6.12}

\section{Transition From Claims to Electronic Health Record Data in National Health Care Research Within the Hospital Setting}

\section{Sonja Williams ${ }^{1}$ \\ ${ }^{1}$ National Center for Health Statistics, Hyattsville, MD}

Background: The National Hospital Care Survey (NHCS) is a survey of hospital care in the United States designed to provide reliable health care utilization data for hospital inpatient and ambulatory settings. National estimates are not yet possible because of low hospital response rates. NHCS collects a year's worth of hospital visits from a sample of hospitals. Since 2011, NHCS collected Uniformed Bill (UB)-04 claims data. Claims data collect up to $25 \mathrm{ICD}$ diagnosis and procedures codes. The diagnosis codes are optimized for billing purposes and are not always reflective of the entirety of the hospital visit. In an effort to address the known limitations of claims data, the NHCS began collecting electronic health records (EHR) in addition to claims, with the ultimate goal of all data being collected from EHR systems. The NHCS's transition from UB-04 claims data to EHR data can provide insight on the successes and challenges of using hospital data in health care research.

Methods: In 2016, most participating hospitals provided either UB-04 data $(n=89)$ or EHR data $(n=31)$. Data were extracted and integrated into a final dataset.

Results: Available data elements varied widely between UB-04 claims hospitals and EHR hospitals. UB-04 claims data provided most information needed to create estimates of care in the hospital but lacked clinical note information. Claims data have no information on medications and labs conducted. Most claims visits $(\sim 87 \%)$ are missing race or ethnicity data. Some EHR hospitals do not have an identifiable hospital setting. In addition, some EHR records do not include diagnoses information and most are missing procedures.

Conclusion: Although claims data are standardized, there are known limitations, namely unreliable race and ethnicity data and no clinical note information that can give more knowledge about the hospital stay. EHR are rich with contextual information such as clinical notes but are based on open standards that vary by EHR source, EHR vendors, and hospital preferences and needs. The results from this study will inform future data collection and integration efforts of EHR and claims data in support of health care research. 
P6.13

Exploration of Differences Between Medication Dispense Data and Pharmacy Claim Data in Estimating Proportion of Days Covered for Statins

Satish Mudiganti, ${ }^{1}$ Xiaowei Yan, ${ }^{1}$ J.B. Jones, ${ }^{1}$ Hannah Husby, ${ }^{1}$ Jake Delatorre-Reimer, ${ }^{1}$ Farah Refai, ${ }^{1}$ Karen MacDonald ${ }^{2}$

${ }^{I}$ Sutter Health, Walnut Creek, CA; ${ }^{2}$ AstraZeneca, Wilmington, $D E$

Background: Pharmacy claims data are the gold standard for assessing adherence, but most health care systems do not have access to these data in a way that supports point-of-care management. Medication dispense data (MDD) identify medications filled at pharmacies, regardless of claims status. As MDD become increasingly available in electronic health records, it is important to understand how they compare to claims data for assessing adherence, which we explore in this study.

Methods: We completed a retrospective analysis from January 1, 2017, to December 31, 2017, on claims and MDD for primary care patients with dyslipidemia who were $\geq 35$ years old, enrolled in a managed care program in an open health care system, and had evidence of statin medication dispenses in the electronic health records. The proportion of days covered (PDC) was calculated for each patient using each data source. Patients were divided into 3 groups: equal (claims PDC = MDD PDC); higher-MDD PDC; and higher-claims PDC. A logistic regression model was applied to estimate how demographics and the number of statins dispensed from claims were associated with each group.

Results: Among 1035 patients (average age: 58 years), $64 \%, 56 \%$, and $79 \%$ were white, male, and non-Hispanic, respectively. The equal group (mean PDC: 0.8 [standard deviation (SD): 0.3]) comprised 566 (55\%) patients, with 9505 and 8833 dispenses recorded in MDD and claims data, respectively. Patients with more dispenses (31-40) were more likely to have the same PDC from both claims and MDD (odds ratio [OR]: 1.7, 95\% CI: 1.1-2.4) compared with reference level (1-10 dispenses). There were 279 (27\%) patients in the higher-MDD PDC group, with significantly fewer dispenses compared with the equal group (5562 MDD and 4078 claims dispenses). PDC mean value was 0.8 (SD: 0.2 ) for MDD and 0.6 (SD: 0.2) for claims. Patients with low dispenses (1-10) were more likely to have higher PDC from MDD compared with higher number of dispenses (31-40) (OR: 2.4, 95\% CI: 1.4-4.0).

Conclusion: A surprising $27 \%$ of patients had higher PDC derived from MDD than claims data. This difference could not be explained by demographic factors but was significantly associated with a lower number of dispenses. Additional studies are needed to understand the differences and quality of MDD PDC measure.

\section{Abstract P6.14 withdrawn by authors after initial publication}

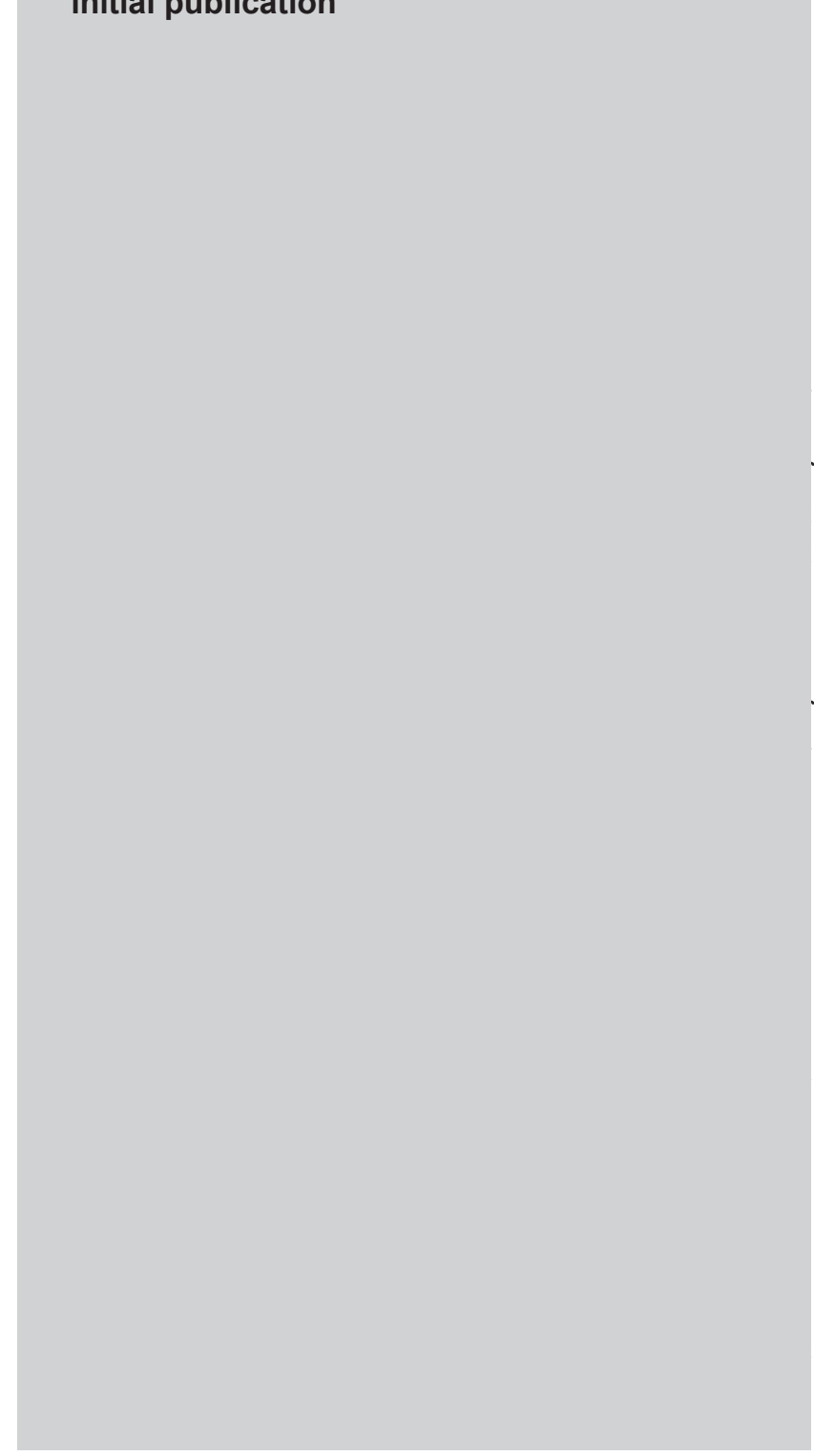

\section{HEALTH SERVICES DELIVERY, UTILIZATION, AND COVERAGE}

OA6.01

Implementation and Effectiveness of Two Models of Direct-to-Member-Mailed Colorectal Cancer Screening Outreach for Medicaid and Medicare Enrollees

Beverly B. Green, ${ }^{1}$ Imara West, ${ }^{2}$ Malaika Schwartz, ${ }^{2}$ Jennifer Coury, ${ }^{3}$ William Vollmer, ${ }^{4}$ Jean Shapiro, ${ }^{5}$ Amanda Petrik, ${ }^{4}$ Laura-Mae Baldwin, ${ }^{2}$ Gloria Coronado ${ }^{4}$ 
${ }^{1}$ Kaiser Permanente Washington, Seattle, WA; ${ }^{2}$ University of Washington, Seattle, WA; ${ }^{3}$ CareOregon, Portland, OR; ${ }^{4}$ Kaiser Permanente Northwest, Portland, OR; ${ }^{5}$ Division of Cancer Prevention and Control, Centers for Disease Control and Prevention, Atlanta, GA

Background: Colorectal cancer screening rates are low and particularly low among individuals enrolled in Medicaid. Little is known about the success of Medicaid health plans' direct-tomember outreach to raise rates of colorectal cancer screening.

Methods: We conducted a hybrid implementationeffectiveness study that compares the implementation and effectiveness of two program models that health plans developed for a mailed fecal immunochemical test (FIT) intervention. The health plans mailed FITs to the homes of ageeligible Medicaid and Medicare health plan members overdue for colorectal cancer screening. One health plan (Health Plan A) used a collaborative approach of mailing co-branded introductory letters, FIT kits, and reminder postcards along with relying on clinics' standard procedures for ordering and processing completed FIT kits; the other health plan (Health Plan B) used a centralized approach of mailing FITs directly to patients, processing them at a centralized laboratory, and delivering live reminder phone calls to patients who had not completed their FITs. We report the implementation and effectiveness of these two models using activities delivered and FIT return rates overall and for member subgroups.

Results: A total of 2812 enrollees in Health Plan A and 8551 enrollees in Health Plan B were mailed an introductory letter in the first year of the program; 2650 (94\%) and 8551 (100\%), respectively, were mailed a FIT kit. Overall, FIT return rates were $17.4 \%$ in Health Plan $\mathrm{A}$ and $18.2 \%$ in Health Plan B. Partnering clinics in Health Plan A carried out a variety of activities to support the program, including reviewing lists of patients prior to the mailing, delivering live phone call reminders, and offering patient incentives for FIT completion; screening rates appeared highest in clinics that reviewed the lists prior to the mailing. Among enrollees in Health Plan B, 9.8\% (839 of 8551) of those eligible received a reminder phone call; return rates were higher for those who received the reminder phone call compared to those who did not $(76.2 \%$ vs $11.9 \%)$.

Conclusion: Our findings may help health plan decision-makers choose between models of delivering mailed FIT outreach that work best for their unique populations and organizations.

\section{OA6.02}

\section{Utilization of Post-Acute Hospitalization Settings in a Sequence of Care in Medicare Beneficiaries}

\author{
Amol Karmarkar, ${ }^{1}$ Yong-Fang Kuo, ${ }^{1}$ Chih-Ying Li, ${ }^{1}$ Amit \\ Kumar, $^{2}$ Kenneth Ottenbacher ${ }^{1}$
}

${ }^{1}$ University of Texas Medical Branch, Galveston, TX; ${ }^{2}$ Northern Arizona University, Phoenix, AZ
Background: The objective of this study was to examine utilization of inpatient rehabilitation facility (IRF), skilled nursing facility (SNF), and home health care (HHA) in posthospital 90-day time period for Medicare beneficiaries. Methods: The study was a secondary analysis of $100 \%$ Medicare claims data (2012-2014). We used data files that linked hospitals and post-acute hospitalization claims, patient-level assessment associated with post-acute hospitalization, provider-level information, and market-level variables. Our study cohort was comprised of cases with index acute hospitalization for 3 conditions: stroke (ischemic and hemorrhagic), lower extremity fractures (hip fractures and other fractures), and lower extremity joint replacements (hip and total knee replacements) $(\mathrm{N}=887,754)$.

Results: For stroke, approximately $31 \%$ of patients did not receive any post-acute hospitalization care, followed by IRF (27.4\%) and SNF (26.2\%). For lower extremity fractures, more than $70 \%$ of the patients went to SNF after acute hospitalization, followed by approximately $21 \%$ going to IRF. For lower extremity joint replacements, $40 \%$ were discharged to SNF, followed by $35 \%$ to HHA. For all the conditions, HHA was utilized both as an index post-acute care setting (after acute hospitalization) and as a subsequent postacute care setting (after SNF or IRF stay). We computed and plotted "dominant patterns" for post-acute care utilization, by conditions and by hospital referral regions, to examine regional variation in utilization of post-acute care. For lower extremity fractures and lower extremity joint replacements, we found significant variation in utilization of post-acute care by hospital referral regions, which is indicative of nonclinical factors influencing discharge planning and delivery of post-acute care.

Conclusion: With so much attention given to episode-based payment models (bundled payment models), it is important to take into account the transition of patients between the post-acute care settings to ensure such transition in a continuum are done to actually improve health outcomes for patients rather than to avoid the unintended consequences (hospital readmission) in a given time period, which can result in a financial penalty to providers. Future work needs to risk adjust for case severity, provider networks (relationship of acute and post-acute care settings), and regional level factors to control for underlying differences to make unbiased comparison of outcomes across different patterns of post-acute care utilization.

\section{OA6.03 \\ Lean Redesigns and Time Working Among Primary Care Physicians}

Dorothy Hung, ${ }^{1}$ Su-Ying Liang, ${ }^{1}$ Quan Truong, ${ }^{1}$ Harold S. Luft ${ }^{1}$

${ }^{1}$ Palo Alto Medical Foundation Research Institute, Palo Alto, $C A$ 
Background: As primary care continues to face high patient demand and provider burnout, health systems are increasingly turning to interventions that improve work efficiency. While research points to the effectiveness of Lean methodology, few studies examine its impact on the amount of time physicians spend engaged in either direct or indirect patient care activities. We used time-stamped electronic health record (EHR) data to reconstruct daily physician time working over the course of 6 years. With these longitudinal data, we assessed impacts of Lean on physician work patterns in a large ambulatory care delivery system.

Methods: This study was based on EHR capture of 15 million daily transactions among 316 primary care physicians over 6 years. We used a stepped-wedge study design and interrupted time series analysis to examine effects of Lean redesigns on daily physician work time. Redesigns included standardizing equipment and patient education materials in all examination rooms, co-locating physician and medical assistant (MA) dyads in a shared workspace, and creating new workflows for care teams. These workflows included care team huddles, agenda setting by MAs at the start of patient visits, and joint management of the electronic inbox. Results: We found a $6.6 \%$ reduction in the amount of time that physicians spent in the EHR each day after the last patient visit $(\mathrm{P}<0.001)$. Additionally, there was a small but statistically significant $1.1 \%$ decrease in hours that physicians continued to work in the clinic or remotely each day, beyond the time spent caring for patients during office visits $(\mathrm{P}<0.001)$. These decreases were observed immediately following the introduction of Lean redesigns and were sustained over a continuous 3-4-year period following Lean intervention. We found no change in number of hours that physicians spent caring for patients during scheduled office visits.

Conclusion: Findings align with previous research on Lean efficiencies in primary and specialty care. Specifically, we found the most benefit in the alleviation of physician time spent on indirect patient care and administrative duties. A lack of observed impact on daily hours spent in office visits suggests that Lean did not adversely impact time allocations for providing direct patient care.

\section{OA6.04}

Leveraging the Electronic Medical Record for ValueBased Improvement and Physician Engagement

\section{Vivian Wu, ${ }^{1}$ Steven Chang ${ }^{1}$ \\ ${ }^{1}$ Henry Ford Health System, Detroit, MI}

Background: Electronic health records have significantly changed health care delivery for both physicians and patients. However, the full potential of the data has not been utilized by the health care system. The barriers to using the available data resources to their full extent are complex. Henry Ford Cancer Institute (Detroit, MI) has developed a methodology to leverage the current available data resources to engage physicians in increasing the value (ie, cost/outcomes) of health care provided. The objective of this study was the development of a comprehensive real-time data reporting mechanism for physicians to understand their clinical practices.

Methods: A multidisciplinary team of hospital administrators, analysts, statisticians, performance improvement staff, and clinicians were brought together to develop a comprehensive real-time data reporting structure. Specific data elements included were volumetric data based on cancer stage, outcomes (both patient-reported and survival relative to national benchmarks), process metrics, financial data, and patient safety data.

Results: The development of the dashboards resulted in significant improvement in many key quality indicators for the health system, including readmission, hospital-acquired infections, and length of stay. Key findings included that data transparency drove clinician engagement into the performance and quality improvement efforts. Engagement has driven constant quality improvement cycles and continued improvement in patient care.

Conclusion: The electronic medical record can be mined to identify key performance indicators that can inform on processes that may require operational changes. Real-time reporting and transparency can provide the fuel for valuebased improvement as our health care systems move away from fee-for-service to fee-for-value.

\section{OA10.01}

Building Multisector Relationships for Health Care Innovation: Network Analyses of Regional Collaborations

\section{Tessa Heeren ${ }^{1}$}

\section{${ }^{1}$ Public Policy Center, University of Iowa, Iowa City, IA}

Background: The purpose of the State Innovation Model (SIM) grant in Iowa is to support the development and execution of innovative plans to achieve better quality of care, lower costs, and improve health for a state's population. One component of the Iowa SIM is the Community and Clinical Care (C3) Initiative, which awarded 7 regions funding to develop community-based coalitions across medical, public health, and social service delivery systems to provide enhanced care coordination for patients. Network analyses were conducted to evaluate how collaborative relationships developed within each of the 7 regions of the $\mathrm{C} 3$ initiative.

Methods: An online survey was administered to organizations involved in the $\mathrm{C} 3$ to gather perspectives about relationship development and collaborative endeavors. Respondents were asked to report about involvement in 3 types of collaborative activities: 1) care coordination; 2) data sharing; and 3) resource sharing. To learn more about the $\mathrm{C} 3$ role in stimulating and maintaining collaboration, respondents were asked about the strength and perceived sustainability of 
relationships. UCINet software was used to analyze networks and identify key organizations within networks.

Results: The networks captured were diverse and engaged stakeholders in clinical health care settings, public health, social services, community programs, insurers, and government entities. The C3 grant had varying degrees of impact across sites. Sites that reported robust collaborative networks before the grant had lower rates of attributing collaborative relationships to the C3. Perceptions of sustainability were promising; across sites, respondents expected around $39 \%$ of the current relationships to continue beyond the funded period. Overall, relationships across sites were rated highly, with nearly half of all reported relationships across $\mathrm{C} 3$ sites receiving the strongest possible rating. Network development varied by activity type, as indicated by the average densities of networks. Across all C3 sites, the most developed networks were those involved in resource sharing, followed by care coordination, and data sharing networks were substantially less developed.

Conclusion: The $\mathrm{C} 3$ initiative was integral to the development of local networks and fortified relationships that are collaborative, multifaceted, cross-sector, and sustainable. Organizations took advantage of the opportunity to share resources with collaborative partners, while establishing data sharing networks has been challenging.

\section{OA10.02 \\ Facility-Specific Barrier Assessment to Guide Local Adaptation of Intervention Strategies to Increase Human Papillomavirus Vaccination}

Chunyi Hsu, ${ }^{1}$ Chun Chao, ${ }^{1}$ Nancy Cannizzaro, ${ }^{1}$ Bhanuja Dub, ${ }^{1}$ Visanee Musigdilok, ${ }^{1}$ Brian Mittman ${ }^{1}$

\section{${ }^{1}$ Kaiser Permanente Southern California, Pasadena, CA}

Background: Human papillomavirus (HPV) is the causative agent for cervical, penile, and anal cancers, among others. HPV vaccines are effective in preventing incident infection and precancerous lesions in HPV-naïve individuals. Centers for Disease Control and Prevention recommends routine HPV vaccination for boys and girls 11-12 years of age. However, despite significant vaccination benefits and evidence-based recommendations, HPV vaccination rates remain suboptimal. Studies show that barriers for HPV vaccination are multifactorial and multilevel and may be heterogeneous across clinics. We conducted a systematic barrier assessment in two Kaiser Permanente Southern California (KPSC) pediatric clinics as part of a pilot study to evaluate tailored interventions to improve vaccination rates. Methods: Two KPSC clinics with significant vaccination gaps were selected. A focus group of providers in one clinic generated an initial list of barriers, augmenting barriers identified through a comprehensive literature review. Barriers were grouped as system-, parent- and providerlevel barriers and incorporated in an online survey $(4,16$, and 9 items, respectively) sent to 125 providers (physicians, nurses, and administrative leads). Respondents were asked to rate barriers as either very important, moderately important, or not important.

Results: Completed surveys were received from 5 physicians and 2 nurses in one clinic (58\% response rate) and 21 physicians, 16 nurses, and 8 online leads in the other $(43 \%$ response rate), for a total of 52 surveys returned. Four of the top five barriers were consistent between the two clinics and were each related to parent knowledge and attitude (vaccine not required by school, not understanding the benefit of the vaccine, wanting it deferred to a later time, and believing the child not at risk). One of the clinics also identified a systemlevel barrier (competing priorities for physicians and nurses) as a top barrier. In one clinic the physicians identified parentlevel barriers as top five, whereas the nurses identified one provider-level barrier (physician's perception of parental concerns) and four parent barriers.

Conclusion: Provider-identified barriers to HPV vaccination differed modestly across two clinics and, within one, between physicians and nurses. Tailoring interventions to address identified local barriers may enhance success in improving vaccination rates.

OA10.03

Using the Electronic Health Record to Identify Patient-Level Factors Related to Missed Opportunities for Human Papillomavirus Vaccine Among Youth 11-13 Years of Age

Christine Joseph, ${ }^{1}$ Lois Lamerato, ${ }^{1}$ Amy Tang, ${ }^{1}$ Vivian Wu, ${ }^{1}$ Charles Barone, ${ }^{1}$ Gwen L. Alexander ${ }^{1}{ }^{1}$ Linda Kaljee, ${ }^{1}$ Paul Kilgore ${ }^{2}$

${ }^{1}$ Henry Ford Health System, Detroit, MI; ${ }^{2}$ Wayne State University, Detroit, MI

Background: The 2006 introduction of a vaccine to prevent infection with the sexually transmitted human papillomavirus (HPV) has engendered much controversy. HPV vaccine coverage in the United States is $53.5 \%$ for age-eligible youth. This is well below the Healthy People 2020 target of $80 \%$ coverage. "Missed opportunities" (patient was eligible and due to receive the vaccine and a dose was not administered) is a major contributor to low rates for Gardasil ${ }^{\circledR}$ 9, a 9-valent HPV vaccine (9vHPV). We used our electronic health record, which is linked to the statewide immunization registry, to describe patient-level factors associated with missed opportunities and for series completion of $9 \mathrm{vHPV}$.

Methods: We retrieved all clinical encounters for youth 11-13 years of age presenting to Henry Ford Health System pediatric or family medicine clinics in 2016. Analyses were restricted to clinics seeing more than 100 age-eligible patients/year and to age-eligible patients for whom there was no evidence of diphtheria, tetanus, pertussis (Tdap), 
meningococcal vaccine (MenACWY), or $9 \mathrm{vHPV}$ in the previous 12 months.

Results: A total of 7724 youth 11-13 years of age made visits during the period under study and had no evidence of previous Tdap, MenACWY, or 9vHPV (49.5\% female; mean age: 11.7 [standard deviation: 0.8]; 60.1\% white, 23.4\% African American). Of the 2187 (28.3\%) receiving Tdap and/or MenACWY during the observation year, 52\% did not receive 9vHPV. Factors associated with missed opportunities for initiation of 9vHPV included older age (odds ratio: 1.2 [95\% CI: 1.0-1.5] for every year increase), white race (1.5 [1.2-1.8]), commercial insurance (1.7 [1.42.0]), and suburban residence (1.8 [1.4-2.3]; all $\mathrm{P}<0.05$. Factors associated with missing the second dose, however, were older age (1.5 [1.1-2.0]), nonwhite race (1.4 [1.0-1.9]), and noncommercial insurance (1.3 [1.0-1.7]).

Conclusion: In this sample, overall adolescent vaccine coverage was low and uptake of $9 \mathrm{vHPV}$ was considerably less than the targeted $80 \%$. Our examination of patient-level factors revealed a patient profile for missed opportunities that is somewhat different from that of missing the second dose. Targeted strategies might account for this difference. Electronic health records linked to statewide immunization registries can help health care systems identify subgroups for whom missed opportunities for administering the $9 \mathrm{vHPV}$ are more prevalent.

\section{OA10.04}

\section{Budgeting to Breathe: Strategies Families Use to Manage Asthma Care Costs}

Lauren A. Cripps, ${ }^{1}$ Melissa B. Gilkey, ${ }^{2}$ Deidre V. Washington, ${ }^{3}$ Kathryn M. Przywara, ${ }^{3}$ Alison A. Galbraith $^{1}$

${ }^{I}$ Center for Healthcare Research in Pediatrics, Harvard Pilgrim Health Care Institute and Harvard Medical School, Boston, MA; ${ }^{2}$ Gillings School of Global Public Health, University of North Carolina, Chapel Hill, NC; ${ }^{3}$ Asthma and Allergy Foundation of America, Landover, $M D$

Background: In the United States, the annual cost of managing asthma has risen dramatically in recent years to over $\$ 3000$ per person on average. Families affected by asthma report difficulty adhering to care regimens because of the high cost of preventive medications and other care coupled with increased cost sharing required by some insurance plans. To inform efforts to reduce asthma cost burden and improve medication adherence, we conducted a qualitative study to explore how commercially insured families affected by asthma manage the cost of care.

Methods: In 2018, we conducted semi-structured telephone interviews with a national sample of 61 commercially insured adults who had asthma and/or were the parent of a child with asthma. Our purposive sample included participants with high-deductible health plans and traditional plans with low or no deductibles. Using a standardized codebook, we analyzed data qualitatively via content analysis.

Results: Across health plan types, participants reported three primary types of consumer behavior for managing asthma care costs. First, participants budgeted for asthma care. Strategies included planning for asthma care in the household budget, putting aside funds in a health-related savings account, or "stockpiling" medications received at no or low cost upon meeting an annual deductible. Second, participants shopped for care to reduce costs. Strategies included comparing medication prices across pharmacies, searching for medication coupons, asking health care providers for samples, and working with providers to identify lower-cost medications. Finally, participants sought to minimize the amount of care they used. For some, this meant avoiding asthma triggers and adhering strictly to preventive medication regimens to avoid costly acute care visits. For others, it meant forgoing preventive medications, sharing them with others, or replacing them with essential oils or other unproven alternative therapies.

Conclusion: Findings of this national study suggest that families affected by asthma are highly engaged as consumers of health care in ways that have both positive and negative implications for medication adherence. Our typology of consumer behaviors can inform efforts to support families in selecting and adhering to high-value care plans while seeking to protect them from the cost burdens that can lead to forgone care.

P9.02

Defining and Characterizing Frequent Attenders: A Systematic Literature Review and Recommendations

\section{Erik Faber, ${ }^{1}$ Dip Shukla, ${ }^{1}$ Brian Sick ${ }^{1}$}

${ }^{1}$ University of Minnesota Medical School, Minneapolis, MN

Background: To decrease cost and improve efficiency, health care organizations have focused on frequent attenders (patients with inappropriately increased health care utilization). Prior studies of this population have investigated singular health care settings, rather than whole health care systems, and they use inconsistent definitions of frequent attendance. Furthermore, there is variation across studies in the factors correlated with frequent attendance. The purpose of this article is to suggest a uniform definition of frequent attendance, including whether it should vary across different health care settings, and to determine the factors correlated with frequent attendance.

Methods: This systematic review of 3 databases found 1835 unique articles, of which 140 met inclusion criteria. These studies were analyzed for their definition of frequent attendance and the factors they found associated with frequent attendance.

Results: Most studies ( $\mathrm{n}=108)$ defined frequent attendance 
by number of health care visits within a set time period, whereas 34 used a top percentile cut-off. This definition varied across primary care, emergency room, and inpatient settings. Based on averages across studies, we propose the following definitions for frequent attenders: for primary care setting, either the top 10th percentile or at least 11 visits in 12 months; for emergency room setting, at least 5 visits in 12 months; and for inpatient setting, at least 4 admissions in 12 months. Common factors correlated with frequent attendance were mental health, drug/substance abuse, and chronic disease.

Conclusion: We propose a definition of frequent attendance for three common health care settings: primary care, emergency room, and inpatient. Furthermore, future studies should include mental health, drug/substance abuse, and chronic disease as factors when studying this population. Adoption of these recommendations will allow comparisons across studies such that meta-analyses could better determine the best interventions for more appropriate utilization of health care resources.

\section{P9.03}

\section{Trends in Medical Imaging From 1996 to 2016 in Pregnant Women in North America: The Radiation- Induced Cancers Study}

Marilyn Kwan, ${ }^{1}$ Diana Miglioretti, ${ }^{2}$ Emily Marlow, ${ }^{2}$ Erin Bowles, ${ }^{3}$ Sheila Weinmann, ${ }^{4}$ Kamala Deosaransingh, ${ }^{1}$ Stephanie Cheng, ${ }^{5}$ Joseph DeVault, ${ }^{6}$ Melanie Francisco, ${ }^{4}$ Matthew Lakoma, ${ }^{7}$ Joanne Mohr, ${ }^{8}$ M. Kay Theis, ${ }^{3}$ Julie Munneke, ${ }^{4}$ Charisma Jenkins, ${ }^{4}$ Casey Luce, ${ }^{3}$ Deborah Multerer, ${ }^{6}$ Prachi Chavan, ${ }^{9}$ James Duncan, ${ }^{10}$ Wesley Bolch, ${ }^{11}$ Lawrence Kushi, ${ }^{1}$ Alanna Rahm, ${ }^{12}$ Robert T. Greenlee, ${ }^{6}$ Natasha Stout, ${ }^{7}$ Jason Pole, ${ }^{13}$ Rebecca Smith-Bindman ${ }^{9}$

${ }^{1}$ Kaiser Permanente Northern California, Oakland, CA; ${ }^{2}$ University of California, Davis, Davis, CA; ${ }^{3}$ Kaiser Permanente Washington Health Research Institute, Seattle, WA; ${ }^{4}$ Kaiser Permanente Northwest, Portland, OR; ${ }^{5}$ Institute for Clinical Evaluative Sciences, Toronto, Canada; ${ }^{6}$ Marshfield Clinic, Marshfield, WI; ${ }^{7}$ Harvard Pilgrim Health Care, Boston, MA; ${ }^{8}$ Kaiser Permanente Hawaii, Honolulu, HI; ' University of California, San Francisco, San Francisco, CA; ${ }^{10}$ Washington University in St. Louis, St. Louis, MO; ${ }^{11}$ University of Florida, Gainesville, FL; ${ }^{12}$ Geisinger Health, Danville, PA; ${ }^{13}$ Pediatric Oncology Group of Ontario, Toronto, Canada

Background: Over the last 20 years, the use of advanced medical imaging has increased dramatically. Medical imaging improves diagnosis of disease and injury, yet many modalities expose patients to carcinogenic ionizing radiation. Rates of imaging in pregnant women, where the risks to the fetus are particularly detrimental, are unknown.

Methods: We evaluated patterns of medical imaging (computed tomography [CT], magnetic resonance imaging [MRI], angiography and fluoroscopy [fluoroscopy], conventional radiography [x-rays], and nuclear medicine [NUCS]) during pregnancy from 1996 to 2016 in 6 U.S. integrated health care systems and Ontario, Canada. Imaging utilization was ascertained using standardized diagnosis, procedure, and billing codes mapped to each imaging modality. We calculated per pregnancy imaging rates stratified by country and birth year using marginal standardization.

Results: In the United States, CT rates increased from 2.0 to 11.4 exams/1000 pregnancies from 1996 to 2007 and declined to $9.3 / 1000$ in 2016. In Ontario, growth in CT rates was less than in the United States, increasing from 2.0/1000 in 1996 to $4.5 / 1000$ in 2007 to $6.2 / 1000$ in 2016. Magnetic resonance imaging (MRI) rates increased steadily and surpassed CT rates in 2013 in the United States $(9.9 / 1000$ vs $9.5 / 1000$, respectively) and in 2007 in Ontario (5.4/1000 vs $4.7 / 1000$, respectively). NUCS rates decreased in the United States from 2004 forward but increased in Ontario to 2.5-fold higher than the United States by $2016(3.3 / 1000$ vs $1.3 / 1000)$. X-ray rates decreased in the United States from $72.6 / 1000$ in 1999 to $47.6 / 1000$ in 2016 but increased in Ontario from 36.2/1000 in 1996 to 44.8/1000 in 2016. Similarly, fluoroscopy rates decreased in the United States from 4.1/1000 in 2001 to $2.4 / 1000$ in 2016 and increased in Ontario from 2.6/1000 in 2005 to 5.2/1000 in 2016.

Conclusion: Overall rates of CT and MRI are higher in the United States than in Ontario. However, the United States had more recent decreases in CT use and increases in MRI use in the last 5 years, while Ontario had gradual increases in both CT and MRI use within the last 10 years. It is important to continue monitoring imaging rates in pregnant women to avoid unnecessary testing and radiation exposure.

\section{P9.04}

\section{A Predictive Model for Chaplain Taxonomy Patterns Identified Through Latent Class Analysis Among Newborns in Health Care Delivery}

Xiaobo Zhong, ${ }^{1}$ Madhu Mazumdar, ${ }^{1}$ Vansh Sharma, ${ }^{2}$ Lina Jandorf, ${ }^{3}$ Denise Welsh, ${ }^{4}$ Deborah B. Marin ${ }^{2}$

${ }^{1}$ Institute for Healthcare Delivery Science, Department of Population Health Science and Policy, Icahn School of Medicine at Mount Sinai, New York, NY; ${ }^{2}$ Center for Spirituality and Health, Department of Psychiatry, Icahn School of Medicine at Mount Sinai, New York, NY; ${ }^{3}$ Center for Behavioral Oncology, Department of Population Health Science and Policy, Icahn School of Medicine at Mount Sinai, New York, NY; ${ }^{4}$ Center for Spirituality and Health, Icahn School of Medicine at Mount Sinai, New York, NY

Background: Several studies describe the nature of chaplains' care, but a major limitation is the lack of a standardized terminology to describe chaplains' activities across different clinical settings. A standard inventory, 
called the Advocate chaplaincy taxonomy, was developed to describe chaplains' activities in 2015 at a tertiary-care teaching facility. The taxonomy includes 100 items divided into 3 categories: intended effects $(n=15)$, methods $(n=26)$, and interventions $(n=59)$. The intended effects category refers to the outcome that a chaplain hopes to accomplish during a visit. Methods describe the manner by which the intended effect is achieved. Interventions refer to what a chaplain does to achieve the desired outcome. The taxonomy was originally suggested to be implemented in an order of intended effect $\rightarrow$ method $\rightarrow$ intervention. Consequently, 23,010 $(15 \times 26 \times 59)$ pathways may result from this order of progression.

Methods: We performed latent class analysis (LCA) to identify underlying taxonomy patterns with newborns in an inpatient setting and built a predictive model.

Results: A 3-class LCA model was selected with pattern 1 showing high probabilities of a collection of 3 intended effects ("Helping someone feel comforted," "Build relationship of care and support," and "Faith affirmation"), with 1 method ("Offer spiritual/religious supports"), and 1 intervention ("Active listening"). Pattern 2 exhibited high probabilities of delivering "Demonstrate caring and concern," "Offer spiritual/religious support," and "Pray," but lower for the other items. The remaining visits were grouped together as a reference pattern. By assigning each newborn to 1 of the 3 identified patterns based on the highest posterior probabilities, we created a categorical latent variable with 133 cases in the reference pattern, 129 in pattern 1, and 559 in pattern 2. Multivariate analysis showed that, compared to newborns in the reference pattern, being a female (odds ratio [OR]: 1.86), a follow-up visit (OR: 3.01), a long-stay visit (OR: 2.67), and department type (NICU/PICU/ED/labor and delivery) were significantly associated with pattern 2 . Meanwhile, a follow-up visit (OR: 4.12) and a long-stay visit (OR: 3.11) were significantly associated with pattern 1 . Conclusion: This is the first study with large sample size (821 visits from 433 patients) and is easily scalable due to its data collection module in Epic electronic medical records.

\section{P9.05}

\section{Effect of Clinical Decision Support on Appropriateness of Advanced Imaging Use Among Physicians-in-Training}

Jashvant Poeran, Lisa J. Mao, ${ }^{1}$ Nicole Zubizarreta, ${ }^{1}$ Madhu Mazumdar, ${ }^{1}$ Bruce Darrow, ${ }^{1}$ Nicholas Genes, ${ }^{1}$ Joseph Kannry, ${ }^{1}$ Paul Francaviglia, ${ }^{1}$ Parley D. Kennelly, ${ }^{1}$ Jayson Whitehorn, ${ }^{1}$ Galen Kilroy, ${ }^{1}$ Darion Garcia, ${ }^{1}$ David S. Mendelson ${ }^{1}$

\section{${ }^{1}$ Icahn School of Medicine at Mount Sinai, New York, NY}

Background: Clinical decision support (CDS) tools have been shown to reduce inappropriate imaging orders. We hypothesized that CDS may be especially effective for house-staff physicians who are prone to overuse of resources.
Methods: Our hospital implemented CDS for computed tomography/magnetic resonance imaging (CT/MRI) orders in the emergency department, with scores based on the American College of Radiology's appropriateness criteria (range of 1-9; higher scores represent more appropriate orders). Data on CT/MRI orders from April 2013 to June 2016 were categorized into pre-CDS (baseline), post-CDS 1 (intervention) with active feedback for scores of $\leq 4$, and post-CDS 2 (intervention) with active feedback for scores of $\leq 6$. Segmented regression analysis with interrupted time series data estimated changes in scores stratified by house staff and non-house staff. Generalized linear models further estimated the modifying effect of the "house staff" variable. Results: Mean scores were 6.2, 6.2, and 6.7 in the preCDS, post-CDS 1, and post-CDS 2 periods, respectively $(\mathrm{P}<0.05)$. In the segmented regression analysis, mean scores significantly $(\mathrm{P}<0.05)$ increased when comparing particularly pre-CDS vs post-CDS 2 for both house staff $(+0.41$ from baseline, 95\% CI: $0.17-0.64)$ and nonhouse staff $(+0.58$ from baseline, 95\% CI: $0.34-0.81)$, demonstrating no differences in effect between the cohorts. The generalized linear model demonstrated significantly higher scores compared to pre-CDS, particularly in postCDS 2 (0.44 increase in scores; $\mathrm{P}<0.05)$. The "house staff" variable did not significantly change estimates in the postCDS 2 period.

Conclusion: Implementation of active decision support increased overall scores of CT/MRI orders. However, there was no significant difference in effect on scores between house staff and non-house staff.

P9.06

Segmentation Based on Disease Burden for Primary Care Populations With Cardiometabolic Conditions

Xiaowei Yan, ${ }^{1}$ J.B. Jones, ${ }^{1}$ Hannah Husby, ${ }^{1}$ Jake Delatorre-Reimer, ${ }^{1}$ Farah Refai, ${ }^{1}$ Frangiscos Sifakis, ${ }^{2}$ Karen MacDonald ${ }^{2}$

${ }^{1}$ Sutter Health, Walnut Creek, CA $;{ }^{2}$ AstraZeneca, Wilmington, $D E$

Background: Population health management depends on segmenting patients with common clinical needs into distinct groups to facilitate targeted services. Historically, segmentation has relied on ad-hoc methods. Longitudinal electronic health records (EHRs) enable data-driven methods to create clinically meaningful segmentation recommendations that explicitly recognize disease burden and severity.

Methods: We completed a retrospective EHR analysis on adult primary care patients, $\geq 35$ years old, with more than 1 cardiometabolic disease (ie, hypertension, hyperlipidemia, diabetes) and 1 related clinical measure (blood pressure, hemoglobin A1c, or low-density lipoprotein cholesterol) during the study period (October 1, 2015-September 30, 
2017) in a large health care system in Northern California. For each patient, we generated a feature vector that included demographic, disease burden, disease complexity, and biometric measures and imputed missing data values using a discriminant function method. To generate reliable clusters, we applied a consensus latent class analysis method and repeated the process for 3-cluster, 4-cluster, and 5-cluster models.

Results: A 5-cluster model was selected based on Akaike information criterion and Bayesian information criterion. Among the 182,884 patients identified, $10.6 \%, 13.6 \%$, $25.4 \%, 22.3 \%$, and $28.2 \%$ were assigned to clusters 1 through 5, respectively. Prevalence of cardiometabolic conditions varied significantly, and 3 clusters had distinct cardiovascular disease (CVD) risk ranges. In cluster 1, the patient mean age was 67 years, average body mass index was 32, 70\% had all 3 cardiometabolic conditions, and $88 \%$ had high CVD risk. Mean age for cluster 2 (66 years) was similar to that of cluster 1 , with most patients having hyperlipidemia (95\%) and 38\% having all 3 cardiometabolic conditions. Cluster 3 was the oldest group (mean age: 69 years), and had a female majority (68\%), a high prevalence of psychiatric conditions (32\% anxiety, 28\% depression), and the highest care utilization (mean primary care visits in 2 years: 8; mean specialist visits: 13). Clusters 4 and 5 were similar in terms of age (59 and 60 years, respectively) and prevalence of diabetes (12\% vs $13 \%)$ and CVD (2\%-3\%).

Conclusion: We used data-driven methods to cluster cardiometabolic patients into more homogeneous subgroups to facilitate individualization of care. We will conduct clinical validation with clinicians to test distinction of clusters, clinical usefulness of segments, and care protocols that meet needs for each cluster.

\section{P9.07}

\section{Clinical Face Validation of a Segmentation Model for Classifying Patients With Cardiometabolic Conditions}

\section{Xiaowei Yan, ${ }^{1}$ J.B. Jones, ${ }^{1}$ Hannah Husby, ${ }^{1}$ Jake Delatorre-Reimer, ${ }^{1}$ Farah Refai, ${ }^{1}$ Frangiscos Sifakis, ${ }^{2}$ Karen MacDonald ${ }^{2}$}

\section{${ }^{1}$ Sutter Health, Walnut Creek, CA $;{ }^{2}$ AstraZeneca, Wilmington, $D E$}

Background: Population health management strategies are important for effective and efficient management of chronic conditions. Many population health management approaches group patients based on measures such as cost; however, data-driven methods based on measures of clinical risk and/or clinical needs have not been extensively evaluated. Such methods can serve as the basis for developing distinct care models for different subgroups of patients, optimizing the way care is personalized to match clinical needs. We conducted a face validation exercise to evaluate the clinical validity of a 5-cluster segmentation model developed using measures of disease burden, complexity, and clinical need. Methods: Akaike and Bayesian information criteria were used to segment 182,884 primary care patients with cardiometabolic conditions into 5 segments. Segmentspecific profiles were created to describe each segment on the basis of decreasing cardiovascular risk (segment 1 to segment 5), demographics, and/or morbidity. Profiles were shared with 3 primary care physicians, and separate semi-structured interviews were conducted focusing on 3 questions: 1) From a patient management perspective, were 5 segments too many or too few to manage? 2) Are the segments clinically distinct? 3) Can a physician correctly assign patients from their panel to the correct segment?

Results: Physicians understood the value of segmentation and believed it will improve patient care, quality, and efficiency if used in a team model. All 3 physicians suggested combining segments 4 and 5 into one segment due to similarities in cardiovascular disease risk and health gaps and believed the other 3 segments have distinct features that distinguish them from each other. All physicians were able to correctly assign patients selected from the highest-risk and the lowest-risk segments, but 2 had difficulty differentiating patients in segment 4 from those in segment 5. Clinicians recommended including clinically relevant and actionable factors for each segment to improve point-of-care utility.

Conclusion: We completed the initial step of establishing the clinical validity of a data-driven segmentation approach derived using measures of disease burden; however, largescale clinical validation with more clinicians is needed to determine the final segments. Future work will focus on refining the description and interpretation of segments and developing segment-specific care models.

P9.09

Five-Year Assessment of Health Care Utilization for Patients With and Without Migraine in a Large Health Care System

Reeti Sharma, ${ }^{1}$ Alice Jacobson, ${ }^{1}$ Shruti Vaidya, ${ }^{1}$ Victoria Chia, ${ }^{2}$ Dawn Buse, ${ }^{3}$ Richard Lipton, ${ }^{3,4}$ Walter Stewart, ${ }^{5}$ Alice Pressman ${ }^{1}$

${ }^{1}$ Sutter Health, Walnut Creek, CA; ${ }^{2}$ Amgen Inc, Thousand Oaks, CA; ${ }^{3}$ Albert Einstein College of Medicine, New York, NY; ${ }^{4}$ Montefiore Medical Center, New York, NY; ${ }^{5} H I N T$ Consulting, Orinda, $C A$

Background: Most evidence on health care utilization of those with migraine is based on self-report and has not been validated by documented use of health care. We describe health care utilization among primary care patients with and without migraine in a large health care system and examine factors that account for variation in use of care.

Methods: Longitudinal electronic health record (EHR) data from Sutter Health in Northern California were obtained on 1.5 million primary care patients between January 1, 2013, 
and December 31, 2017. A previously developed and validated diagnostic algorithm was used to identify 94,149 patients who sought care for migraine and 1.4 million who did not. We stratified migraine patients by those who sought care exclusively from primary care (PC-M) and those who saw a neurologist $(\mathrm{N}-\mathrm{M})$. We extracted counts of encounters for any reason for primary care, neurology, emergency department (ED), and inpatient visits for all patients during the 5-year period.

Results: Compared to primary care patients without migraine, patients with migraine experienced statistically significantly more encounters per patient (EPP) over 5 years for primary care (10.8 migraine vs 6.4 no migraine), neurology (1.2 vs 0.2 ), inpatient ( 0.17 vs 0.11 ), and $\mathrm{ED}(0.67$ vs 0.26$)$ visits $(\mathrm{P}<0.05$ for all). Among migraine patients, N-M had more ED EPP than PC-M (0.86 vs 0.62, $\mathrm{P}<0.001)$. For ED encounters, $\mathrm{N}-\mathrm{M}$ were more likely to have a discharge diagnosis of migraine than PC-M (14\% vs $9 \%, \mathrm{P}<0.001)$.

Conclusion: During the 5-year period, migraine patients averaged higher any-reason EPP compared to those without migraine for all types of encounters assessed, with the majority of visits occurring in the primary care setting. N-M were more likely to have ED encounters, and those encounters were more likely to be for migraine. A limitation to this analysis is that we are unable to distinguish between episodic and chronic migraine patients. It is likely that neurologists see a higher percentage of chronic migraine patients than primary care physicians, and these patients may utilize health care resources more. Further studies are planned to understand factors that contribute to higher health care utilization rates for N-M patients.

\section{P9.10}

\section{A Web-Based Clinical Decision Support System (Wizard ${ }^{\odot}$ ) Increases Appropriate Cardiometabolic Diagnostic Coding at Primary Care Visits}

\author{
JoAnn Sperl-Hillen, ${ }^{1}$ Jeffrey Anderson, ${ }^{1}$ Rebecca \\ Rossom, ${ }^{1}$ Kris Ohnsorg, ${ }^{1}$ Jay Desai, ${ }^{1}$ Heidi L. Ekstrom, ${ }^{1}$ \\ A. Lauren Crain, ${ }^{1}$ Patrick J. O'Connor ${ }^{1}$
}

\section{${ }^{1}$ HealthPartners Institute, Bloomington, $M N$}

Background: Accurate coding of complex patients is essential for care systems to remain viable in a value-based payment world that includes risk-adjustment. The objective of this study was to determine if use of the Wizard ${ }^{\circ}$ clinical decision support (CDS) system that improves quality of care for patients with uncontrolled cardiovascular risk increases appropriate diagnostic coding of cardiometabolic conditions at primary care visits.

Methods: We conducted a clinic randomized trial of a webbased CDS system at 17 primary care clinics in Minnesota from March 15, 2017, to December 31, 2017. The CDS system a) identified adults at primary care encounters with high reversible cardiovascular risk, and prompted nurses to print Wizard handouts and give them to patients and providers immediately antecedent to the visit; b) provided prioritized evidence-based clinical care recommendations for any uncontrolled major cardiovascular risk factors to promote shared decision-making; and c) retained data from eligible encounters at intervention and control clinics in a data repository that was later mapped to electronic health record Clarity data to identify encounter-level ICD-10 diagnostic codes for diabetes, hypertension, hyperlipidemia, smoking, and obesity.

Results: At 34,281 targeted encounters with 18,782 patients with reversible cardiovascular risk, mean age was $57.7,50 \%$ were female, $13 \%$ were black, $6 \%$ Asian, 3\% Hispanic, and $75 \%$ white, mean 10-year atherosclerotic cardiovascular disease (ASCVD) risk was $15.7 \%$, and mean body mass index (BMI) was 33.0. Wizard handouts were printed for $75 \%$ of targeted encounters in intervention clinics. The percentage of encounters with ICD-10 codes identified were (control vs intervention, P-value): diabetes coded if diabetes identified (70.8\% vs $74.5 \%, \mathrm{P}<0.0001)$; hypertension coded if hypertension identified ( $57.9 \%$ vs $60.9 \%, \mathrm{P}<0.0001$ ); hyperlipidemia coded if statin intensification was indicated (29.9\% vs $31.9 \%, \mathrm{P}=0.005)$; ASCVD coded if ASCVD identified $(24.7 \%$ vs $26.8 \%, \mathrm{P}=0.051)$; obesity coded if $\mathrm{BMI}$ was $\geq 30$ ( $14.8 \%$ vs $17.3 \%, \mathrm{P}<0.0001)$; and smoking coded if current smoking identified ( $25.8 \%$ vs $32.4 \%, \mathrm{P}<0.0001$.

Conclusion: This CDS system significantly increased the likelihood that known cardiovascular risk factors were assigned clinically appropriate ICD-10 diagnostic visit codes at primary care encounters.

\section{P9.11}

\section{Prescription Drug Price Variation Among Community Pharmacies in Texas}

\section{Lixian Zhong, ${ }^{1}$ Ashley Parambil, ${ }^{1}$ Niraj Patel, ${ }^{1}$ Toney Duong $^{1}$}

\section{${ }^{1}$ Texas A\&M University, College Station, TX}

Background: Prescription drug prices pose a significant boundary to medication access in uninsured patients in the United States. In Texas, data on retail and independent pharmacies' prescription drug prices impact in communities were limited. This study seeks to evaluate the variation in out-of-pocket prices for different prescription drugs sold in community pharmacies across the state of Texas and to assess demographic, socioeconomic, and disease-state factors that may influence prescription drug prices.

Methods: Telephone interviews were conducted to each pharmacy to collect listed cash prices for 5 common prescription drugs (levothyroxine $25 \mathrm{mcg}$, lisinopril $10 \mathrm{mg}$, simvastatin $20 \mathrm{mg}$, metformin $500 \mathrm{mg}$, and azithromycin 250 $\mathrm{mg}$ ). Furthermore, county-specific information on population median age, median household income, education level, marital status, the rate of uninsured individuals, obesity rate, the density of pharmacies, and the population density of 254 Texas 
counties were collected to assess factors that may influence prescription drug prices. Descriptive statistics and multivariate regression analysis were performed to describe the price pattern and identify factors associated with price variations.

Results: A random sample of 203 community-independent pharmacies was generated from a list provided by the Texas State Board of Pharmacy. Telephone interviews were administered to the pharmacies listed in the random sample. Of the 203 pharmacies, 144 participated in the interview, with $57 \%$ in metro areas and $43 \%$ in nonmetro areas. The mean price for levothyroxine $25 \mathrm{mcg}$ was $\$ 18.85$ (standard deviation [SD]: \$7.83), for lisinopril $10 \mathrm{mg}$ was $\$ 12.10$ (SD: $\$ 7.85$ ), for simvastatin was 18.71 (SD: $\$ 29.33$ ), for metformin was 14.63 (SD: \$11.43), and for azithromycin was $\$ 20.01$ (SD: \$14.69). We found that drug prices varied significantly across different pharmacies. Multivariate regression model suggested that pharmacy density and population density had statistically significant impact on the drug prices.

Conclusion: We found that drug prices varied significantly across different pharmacies. Our study identified key factors that impact drug prices. Overall, they were positively associated with population density and negatively associated with pharmacy density $(\mathrm{P}<0.05)$.

\section{HEALTH EQUITY AND SOCIAL NEEDS}

\section{OA7.01 \\ Social Needs Programs Across Kaiser Permanente: Findings and Recommendations From an Inventory and Scoping Review}

\section{Andrea Paolino, ${ }^{1}$ Caroline Joyce,,${ }^{1}$ Tina Kimpo, ${ }^{1}$ John Steiner ${ }^{1}$}

${ }^{1}$ Institute for Health Research, Kaiser Permanente Colorado, Denver, $\mathrm{CO}$

Background: Social, economic and behavioral needs (SEBN) are critical determinants of health. Kaiser Permanente (KP) has developed programs to identify and mitigate those needs across its 8 geographic regions, actively collaborating with community organizations that provide basic resources. The KP Social Needs Network for Evaluation and Translation (SONNET) identified these social needs programs and conducted a scoping review that assembles evidence from them.

Methods: In 2017-2018, SONNET identified 33 programs that address basic resource needs. Information about the design, conduct, and impact of these programs was collected through discussions with program leaders and community organizations, qualitative interviews and focus groups with members and front-line staff, member and staff surveys, analyses of data from the electronic health record and other information systems, program evaluations, and published research. Information from these programs was synthesized using a 5-step care continuum framework: 1) plan the assessment and intervention; 2) assess for basic resource needs; 3) connect members to community organizations; 4) improve health outcomes; and 5) spread effective interventions.

Results: SONNET made program information available to KP staff in a searchable database. Evidence from these programs was synthesized into a scoping review that uses a populationbased, community-engaged care continuum model to discuss program findings and make recommendations. Of the 33 programs studied, most target high-risk members (complex care, Medicaid, high-cost members) and assess food, housing, transportation, and medical costs. Moreover, most programs rely on community health workers or patient navigators to connect members to community resources. Only 5 programs had been rigorously evaluated, while 15 had collected some data. Three were conducted in multiple regions, and 30 took place in a single region.

Conclusion: In addition to detailed recommendations at each step of the care continuum, general recommendations for $\mathrm{KP}$ that arise from this scoping review are: set priorities and expectations for needs to assess; prospectively define outcomes to judge the success of interventions; plan systematically to address all steps of the care continuum; include multiple perspectives (members/patients, staff, clinicians, and leaders) in program design; develop the capacity of community organizations; evaluate programs rigorously; design for dissemination; and stay the course since basic resource needs are difficult to resolve and prone to recur.

\section{OA7.02 \\ Assessing the Burden of Social and Economic Needs Among Medicare Enrollees in an Integrated Health Care System}

Matthew Banegas, ${ }^{1}$ Stephanie Fitzpatrick, ${ }^{1}$ John Dickerson, ${ }^{1}$ David Mosen, ${ }^{1}$ Gloria Coronado, ${ }^{1}$ Celeste Machen, ${ }^{1}$ Alexandra Varga, ${ }^{1}$ Rachel Gold ${ }^{1}$

${ }^{1}$ Kaiser Permanente Center for Health Research, Portland, $O R$

Background: Unmet social and economic needs (SEN) influence individuals' ability to make health behavior changes and attain their full health potential. We sought to estimate the prevalence of, and identify characteristics associated with, SEN among Medicare enrollees in an integrated health care system.

Methods: We identified 4504 Kaiser Permanente Northwest members who were $\geq 65$ years of age, were enrolled in Medicare, and had completed a SEN screening assessment, Your Current Life Situation, during a care visit between June 2017 and September 2018. Measures of SEN included financial hardship, food insecurity, housing, and transportation. We estimated the percentage of members who reported experiencing each SEN and any SEN. Separate multivariable logistic regression models were used 
to evaluate the associations between sociodemographic characteristics (age, sex, race, Hispanic ethnicity, median household income, and neighborhood deprivation index), annual maximum out-of-pocket (MOOP) limit, and comorbidity with having each SEN and any SEN.

Results: Among 4504 Medicare members, 25.6\% reported any SEN, 17.2\% financial hardship, $6.7 \%$ food insecurity, $9.0 \%$ housing, and $9.8 \%$ transportation needs. In adjusted models, factors statistically significantly associated with greater odds of having any SEN included younger age $(\mathrm{P}<0.001)$, female sex $(\mathrm{P}<0.001)$, lower income $(\mathrm{P}<0.001)$, African American race $(\mathrm{P}<0.05)$, lower comorbidity burden $(\mathrm{P}<0.001)$, higher MOOP $(\mathrm{P}<0.001)$, and lower neighborhood deprivation $(\mathrm{P}=0.002)$; these findings were generally consistent across each SEN. Specifically, female Medicare members had significantly greater odds of experiencing financial hardship (adjusted odds ratio [OR]: 1.44, 95\% CI: 1.22-1.70), food insecurity (OR: 1.53, 95\% CI: 1.19-1.97), housing (OR: 1.39, 95\% CI: 1.12-1.73), and transportation needs (OR: 1.47, 95\% CI: 1.19-1.80) compared to their male counterparts. African American Medicare members had significantly greater odds of experiencing financial hardship (OR: 1.95, 95\% CI: 1.26-3.03), food insecurity (OR: 2.04, 95\% CI: 1.14-3.64), and housing needs (OR: 2.22, 95\% CI: 1.34-3.69) compared to white Medicare members.

Conclusion: Our study provides valuable insights into the percentage of Medicare members with SEN and characteristics that may help identify those at greater risk of SEN. As efforts to include social factors in risk adjustment for payment models increase (eg, Medicare reimbursement), evaluating the impact of SENs on health care use, costs, and health outcomes across health plan membership subgroups will be imperative.

\section{OA7.03}

\section{The Health of Gender Minorities}

Mark Schmidt, ${ }^{1}$ Dagan Wright, ${ }^{2}$ Denis Nyongesa, ${ }^{1}$ A. Gabriela Rosales, ${ }^{1}$ Allison O'Neill, ${ }^{2}$ Erin Waters ${ }^{3}$

${ }^{1}$ Kaiser Permanente Center for Health Research, Portland, OR; ' $O C H I N$, Portland, OR; ${ }^{3}$ Gender Pathways Clinic, Kaiser Permanente Northwest, Portland, OR

Background: While gender minorities (GM) are known to experience significant disparities compared to their cisgender counterparts, research in this area remains scant. Our goal is to conduct the largest, most comprehensive analysis to date of health metrics recorded in the electronic health record from persons seeking care within Kaiser Permanente Northwest (KPNW) and the OCHIN network of federally qualified community health centers.

Methods: Our study population includes KPNW and OCHIN members, 10-80 years of age, with at least one in-person encounter from January 1, 2012 to December 31, 2017. We have defined GM status based on ICD-9/-10 code, self-reported GM status, encounters with the KPNW Gender Pathways Clinic, referrals for transgender-specific health services, and relevant text strings in the physician note fields. We're assessing differences between GM and cisgender individuals in health care utilization, health behaviors, preventive health screening and vaccination, and a total of 67 validated chronic disease conditions.

Results: Our preliminary analysis included KPNW members and has assessed 27 diagnosed conditions to date. We have identified 1742 GM among a study population of 782,793 $(0.22 \%)$. Compared to cisgender individuals, we found significant, age-adjusted odds ratios (aOR) among GM for benign prostatic hyperplasia (aOR: 0.5 [95\% CI: 0.3-0.9]), breast cancer (0.4 [0.1-0.9]), and depression (3.9 [3.6-4.2]). Borderline significant aOR were found for asthma (1.2 [1.0 1.4]), chronic obstructive pulmonary disease (0.9 [0.7-1.0]), diabetes $(1.2[1.0-1.5])$, hypertension $(0.9[0.8-1.0])$, and rheumatoid arthritis $(0.8[0.6-1.0])$. We also found the GM were more likely to have been screened for depression, using PHQ-9, than their cisgender counterparts (aOR: 1.7 [95\% CI: $1.5-1.8]$ ).

Conclusion: Our preliminary findings are mixed to date, although consistent with a previously reported increase in the occurrence of depression among GM. Analysis of the remaining 40 diagnosed conditions, health utilization, and preventive health measures; the inclusion of data from 5598 GM individuals from the OCHIN study population of $1,961,745(0.29 \%)$; and the assessment of additional covariates will be completed by the end of December 2018 . We will present the most significant health disparities observed overall and across the age spectrum and will further characterize relationships by gender identity and receipt of gender-affirming hormone therapy.

\section{OA7.04}

\section{Development of the PREMM5 Electronic Application for Lynch Syndrome Risk Assessment in a Diverse Population}

Tia Kauffman, ${ }^{1}$ Kathleen Mittendorf, ${ }^{1}$ Chinedu Ukaegbu, ${ }^{2}$ Donna Eubanks, ${ }^{1}$ Jake Allen, ${ }^{1}$ Carmit McMullen, ${ }^{1}$ Nangel Lindberg, ${ }^{1}$ Katrina Goddard, ${ }^{1}$ Sapna Syngal ${ }^{2}$

${ }^{1}$ Kaiser Permanente Center for Health Research, Portland, OR; ${ }^{2}$ Dana Farber Cancer Institute, Boston, MA

Background: Lynch syndrome (LS) is the most common hereditary colorectal cancer syndrome. A comprehensive collection of patient family health history allows for the identification of patients at risk for LS, but rarely is the information collected in sufficient detail. While there are tools to collect targeted family history for risk assessment, these tools require high health literacy or are for providers. These are barriers to genomic medicine access for underserved populations. The PREMM5 model is a validated 
risk assessment algorithm used by health care providers to identify individuals at risk for LS. To improve health care access, we have developed a patient-facing version of PREMM5 for implementation in low-literacy, low-resource populations as part of the CSER2 consortium.

Methods: The PREMM5 developers worked with a team of genetics professionals, researchers, user-experience design experts, software engineers, and patient stakeholders. The patient-facing version was created in both English and Spanish and employs responsive design, allowing for ease of completion on multiple types of electronic devices. The web-based application is available at any time with personal computers or mobile devices.

Results: Two major adaptations in developing the patientfacing application were 1) not relying on the patient to determine the affected side of the family for risk assessment, and 2) guiding the patient through the collection of medical history on each relative to avoid the use of complex terms such as "first-degree relative." Embedded literacy aids, including family tree infographics and pop-up definitions for certain concepts, were accessible via links in the interactive application. Additionally, to optimize the user's experience, skip-logic presented patients with questions relevant to their previous responses. We have a $10 \%$ response rate to an email and text sent to primary care patients; 164 patients have successfully completed the tool, with $27 \%$ scoring at $\geq 2.5 \%$ probability of having an LS mutation. Very few patients requested assistance, and $49 \%$ of respondents met our diversity criteria (racial/ethnic minority or low socioeconomic status).

Conclusion: Patients can complete a lower literacy patientfacing family history risk assessment tool to assess their risk of LS. While our response rate to email outreach is high, our respondents skew toward those more likely to be at high risk.

\section{OA14.01 \\ Health Care Providers' Perspectives on the Opportunities for Mobile Health Technologies to Support Patients' Health Care Needs}

\section{Sharon Laing, ${ }^{1}$ Carlota Ocampo, ${ }^{2}$ Stacey Baugh ${ }^{2}$ \\ ${ }^{1}$ University of Washington Tacoma, Tacoma, WA; ${ }^{2}$ Trinity Washington University, Washington, DC}

Background: Health care providers (HCPs) guide patients and empower them to manage their care; however, patients are becoming more informed from broad-based access to health information. This qualitative study presents HCP perspectives about the value of mobile health technologies to enhance patient-centered care and facilitate trust-building. Methods: Four 60-minute focus group sessions were conducted with medical doctors, nurses, social workers, behavioral health specialists, and community health workers offering services to patients in safety-net settings. Focused topics evaluated were: 1) role of mobile health tools in supporting health; 2) perceived value of an mHealth clinical practice guide to inform patient care; and 3) perceived role of mobile technology in increasing access for diverse ethnic communities. Provider responses were tape-recorded, transcribed, and analyzed for common themes. The method of analysis was evaluating focus group questions for codewords, codewords were then organized into codeword clusters, and then clusters assessed to derive themes/ concepts. Respondents were offered $\$ 75$ for participation.

Results: HCPs saw mHealth tools like tracking devices and health apps as valuable for improving data accuracy and treatment delivery; one respondent noted, "We missed something completely and so let's take a look at that so that I can tailor my treatment to more of the day-to-day instead of what that person is just bringing in on the day that they happen to see me." A clinical practice guide supporting mHealth practices was welcomed by HCPs with the recommendation that such a guide is interactive, simple to use, and presents research-tested mobile devices - "Here is some research behind it, and then that can help them buy in and use it, too." Finally, HCPs perceived value of mobile health technology when working with ethnically diverse communities; they saw the technology as providing a direct link to the patient and provider, thus building trust and improving relationships.

Conclusion: Health care providers recognize the value of mobile health technologies to advance patient outcomes by helping to improve treatment delivery and supporting patient-provider relationships. Providers support an mHealth clinical practice guide with scientific evidence to support recommendation of mobile health technologies.

OA14.02

Understanding the Health Care Needs of Children in Foster Care in Oregon's Community Health Centers

\author{
Frances Lynch, ${ }^{1}$ M.J. Dunne, ${ }^{2}$ Megan Hoopes, ${ }^{2}$ Annie \\ Larson, ${ }^{2}$ Allison O'Neil, ${ }^{2}$ Brigit Hatch ${ }^{3}$
}

${ }^{1}$ Kaiser Permanente Center for Health Research, Portland, OR; ${ }^{2}$ OCHIN, Portland, OR; ${ }^{3}$ Oregon Health \& Science University, Portland, OR

Background: Children in foster care are a vulnerable population. Early life circumstances, including placement in foster care, can predict socioeconomic and health status in adulthood. It is important for medical providers to understand how the health and social needs of children in foster care compare to children in other family arrangements.

Methods: We conducted a case-control study using secondary analysis of electronic health record (EHR) data from Oregon clinic members of the OCHIN community health information network and Medicaid data. We constructed a retrospective cohort of foster children between 6 months and 17 years old in 2014-2016 $(\mathrm{n}=2223)$. We identified a comparison group 
of children from the same clinics meeting the same criteria but without evidence of foster system contact $(n=6304)$. In this descriptive analysis, we compared foster children to their matched controls on a range of demographic characteristics, health care access and utilization measures, and chronic condition and comorbidity burden. All study variables were obtained from the EHR. Time-varying characteristics (eg, federal poverty level, Charlson comorbidity index) were assigned as of each patient's last visit in the study period. We tested for differences between the groups using generalized estimating equation models. These models took on different forms and link functions depending on the distribution of each outcome (normal, binomial, multinomial, or Poisson). Statistical significance was set at $\alpha=0.05$.

Results: Youth in foster care had much greater need, with over $50 \%$ having multiple comorbid chronic health conditions. In particular, youth had many more mental health conditions. Most striking was the prevalence of depression, anxiety, attention-deficit/hyperactivity disorder, and posttraumatic stress disorder in youth in foster care. Youth in foster care have greater service use in some categories, such as mental health (mean: 5.6 visits [standard deviation (SD): 5.0] vs non-foster care mean: 2.3 visits [SD: 10.9]), and less use in other categories, such as dental care (mean: 0.7 visits [SD: 1.8] vs non-foster care mean: 1.0 visits [SD: 2.4$]$ ).

Conclusion: Youth in foster care have much greater need than comparable youth served in community health clinics. However, many may not receive as many services as needed.

\section{OA14.03}

\section{Neighborhood Socioeconomic Disadvantage Is Associated With Multimorbidity in a Large, Geographically Defined Population}

\begin{abstract}
Alanna Chamberlain, ${ }^{1}$ Lila Finney Rutten, ${ }^{1}$ Patrick Wilson, ${ }^{1}$ Chun Fan, ${ }^{1}$ Walter Rocca, ${ }^{1}$ Jennifer St. Sauver ${ }^{1}$
\end{abstract}

${ }^{1}$ Mayo Clinic, Rochester, $M N$

Background: Approximately 1 in 4 adults in the United States have multimorbidity, and the number of persons with multimorbidity will increase exponentially over time, causing escalating health care costs and burden on the health care system. The prevalence of multimorbidity varies across populations, and one potential source of this variation may be differences in socioeconomic status. Thus, we aimed to study the association of an area-level measure of socioeconomic status at the census-block-group level with multimorbidity in a large, geographically defined population.

Methods: Adults ( $\geq 20$ years of age on April 1, 2015) from 7 counties in southern Minnesota $(\mathrm{N}=198,941)$ were identified using the Rochester Epidemiology Project. Diagnostic codes between April 1, 2010, and March 31, 2015, were used to define the prevalence of 21 chronic conditions. Each patient's geolocation (latitude and longitude) was calculated and spatially linked to census block group $(n=251$ census block groups). For each census block group, 5-year estimates (2011-2015) from the American Community Survey or the 2010 U.S. Census were used to calculate the Singh area deprivation index (ADI), a composite measure of neighborhood socioeconomic disadvantage including 17 census measures. The ADI was divided into quintiles, and modified Poisson regression was used to model associations between ADI and multimorbidity ( $\geq 2$ chronic conditions) after adjusting for age, sex, race, and ethnicity.

Results: The age- and sex-standardized (to 2010 Census) prevalence of multimorbidity ranged from $9.9 \%$ to $36.4 \%$, with a median of $23.4 \%$ across census block groups. Compared with persons in the lowest quintile of ADI, those in the highest quintile had nearly a $20 \%$ increased risk of having multimorbidity (relative risk: 1.19, 95\% CI: 1.17-1.21). There also was evidence of an interaction between age and $\mathrm{ADI}(\mathrm{P}<0.001)$ : after age 70 years, no difference in the risk of multimorbidity was observed across quintiles of ADI.

Conclusion: Higher ADI was associated with increased risk of multimorbidity, with more pronounced associations observed for younger persons.

OA14.04

Re-Evaluation of Race and Ethnicity With Adverse Outcomes in Heart Failure

Samuel Savitz, ${ }^{1}$ Jamal Rana, ${ }^{1}$ Keane Lee, ${ }^{1}$ Sue Hee Sung, ${ }^{1}$ Thomas Leong, ${ }^{1}$ Grace Tabada, ${ }^{1}$ Alan $\mathrm{Go}^{1}$

\section{${ }^{1}$ Kaiser Permanente Northern California, Oakland, CA}

Background: Conflicting evidence exists about the relationships between race and ethnicity with heart failure (HF) hospitalization and death in adults with HF. In addition, it remains unclear to what degree modifiable factors may explain variation in these clinical outcomes, especially when health care access is similar. We examined the association of race/ethnicity and outcomes in a diverse community-based cohort of adults with HF receiving care in a large, integrated health care delivery system.

Methods: We identified all adults with HF, 20052012, within Kaiser Permanente Northern California. Demographics, clinical characteristics, receipt of therapies, and left ventricular ejection fraction were ascertained from electronic health records, and regional workforce and socioeconomic status factors from state data. We conducted Cox proportional hazards models separately for 1) time to first HF hospitalization following diagnosis for index cases or identification for prevalent cases, and 2) all-cause mortality. The models serially adjusted for distinct domains to identify potential confounders and mediators.

Results: We identified 25,386 HF patients (70\% white, 9\% Asian/Pacific Islander (API), 11\% black, 11\% Hispanic). Whites were older (mean: 75 years) than other groups (mean: 69 years for API, 67 years for blacks, and 71 years for 
Hispanics. Blacks had a significantly higher fully adjusted rate of HF hospitalization compared to whites (hazard ratio [HR]: 1.34, 95\% CI: 1.24-1.50), which was not seen with APIs and Hispanics. APIs had significantly lower mortality compared to whites in fully adjusted models (HR: 0.83, 95\% CI: 0.74 0.93), while blacks and Hispanics had similar mortality.

Conclusion: The associations between race/ethnicity and HF hospitalization and death remain complex. Unlike Hispanics and APIs, blacks had a higher rate of $\mathrm{HF}$ hospitalization compared to whites but similar mortality. In contrast, APIs had lower mortality compared to whites but a similar rate of HF hospitalization. The findings of higher HF hospitalizations among blacks and lower mortality among APIs are consistent with prior research. However, we did not replicate the previous findings of lower mortality among blacks and lower HF hospitalizations among APIs.

\section{P8.01}

\section{Asian Ethnic Group Disparities in Health and Behavioral Risk Factors in Kaiser Permanente Northern California}

\author{
Nancy P. Gordon, ${ }^{1}$ Joan C. Lo, ${ }^{1}$ Teresa Y. Lin ${ }^{1}$ \\ ${ }^{1}$ Division of Research, Kaiser Permanente Northern \\ California, Oakland, $C A$
}

Background: The category "Asian" is generally used for reporting race/ethnic differences in health-related measures. This broad "Asian" category may be problematic for service planning and comparing populations with different ethnic group compositions.

Methods: We used data for a cohort of 276,671 Kaiser Permanente Northern California Asian adults 45-84 years of age, $97 \%$ of whom were assignable to an Asian ethnicity. The cohort was restricted to members enrolled all of 2016 and included 88,983 Filipinos, 86,322 Chinese, 8968 Koreans, 17,822 Japanese, 32,483 Southeast Asians, and 35,179 South Asians. We used electronic health record data to produce prevalence estimates age-sex standardized to the 2010 U.S. Census for "Asian" and all ethnic groups: for age 45-84, moderate/severe obesity (body mass index [BMI] of $\geq 35$; Asian-specific BMI of $\geq 32.5$ ), smoking, diabetes, hypertension, hyperlipidemia, and asthma; for age 65-84, coronary artery disease $(\mathrm{CAD})$ and heart failure. $\mathrm{A}>2$-percentage-point (PP) difference between "Asian" and ethnic groups ( $>1$ for smoking, CAD, and heart failure) was considered meaningful. Results: $23.1 \%$ of Asians (age 45-84) had diabetes, $42.7 \%$ hypertension, $44.8 \%$ hyperlipidemia, and $10.2 \%$ asthma; $3.3 \%$ had a BMI of $\geq 35,6.9 \%$ a BMI of $\geq 32.5$; and $5.9 \%$ were current smokers. Among those 65-84 years of age, $5.4 \%$ had $\mathrm{CAD}$ and $4.7 \%$ heart failure. We found significant differences across Asian ethnic groups on all measures except BMI of $\geq 35$ (ranges: $15.4 \%-31.9 \%$ for diabetes; $33.6 \%$ $56.1 \%$ for hypertension; $37.5 \%-47.9 \%$ for hyperlipidemia; $6.1 \%-14.3 \%$ for asthma; $3.6 \%-8.1 \%$ for CAD; $3.5 \%-6.4 \%$ for heart failure; $3.1 \%-10.1 \%$ for BMI of $\geq 32.5$; and $3.0 \%-$ $7.6 \%$ for smoking). On all 6 chronic conditions and BMI of $\geq 32.5$, Filipinos and South Asians were higher than "Asian" and Chinese, while Chinese and Koreans were lower than "Asian" on these conditions. Southeast Asians measured lower than "Asian" on diabetes, hypertension, heart failure, and BMI of $\geq 32.5$, and Japanese were lower on diabetes, hypertension, hyperlipidemia, and heart failure but higher on BMI of $\geq 32.5$. Chinese and South Asians were lower than "Asian" on smoking, while Filipinos, Koreans, and Southeast Asians were higher.

Conclusion: We found disparities across Asian ethnic groups in prevalence of chronic conditions, smoking, and moderate/ severe obesity based on Asian-specific criteria. Filipinos and South Asians have an overall worse chronic-disease and risk-factor profile than East Asians, with exceptions of lower smoking among South Asians and higher obesity among Japanese. Capture of Asian ethnicity in electronic health records is recommended.

\section{P8.02 \\ Use of Geospatial Data for Assessing and Addressing Health Disparities Within a Lung Cancer Screening Population}

\section{Caroline Joyce, ${ }^{1}$ Brian Hixon, ${ }^{1}$ Nikki Carroll, ${ }^{1}$ Andrea Burnett-Hartman, ${ }^{1}$ Debra Ritzwoller ${ }^{1}$}

\section{${ }^{1}$ Institute for Health Research, Kaiser Permanente Colorado, Denver, $\mathrm{CO}$}

Background: Many studies use census-based data to ascertain proxies of education and income; however, these measures are limited because they are not sufficiently granular and therefore do not adequately estimate measures of wealth, access to care (ie, travel time, density of providers), neighborhood deprivation, and other social determinants of health.

Methods: We are using newly available sources of geospatial data to derive more nuanced proxy measures for social determinants of health and health disparities in lung cancer screening. These sources include the Food Access Research Atlas; open-source property data from sources like Zillow, Redfin, and Realtor.com; restricted microdata from the U.S. Census Bureau; the Centers for Disease Control and Prevention's Social Vulnerability Index (SVI); and other data such as maps from the Environmental Protection Agency, The Opportunity Atlas, and The Eviction Lab. By mapping data from these sources to the census-based variables available in our electronic health records (EHR) (eg, FIPS codes, zip codes), we can calculate robust proxies for social determinants of health.

Results: One of the strengths of our study is the heterogeneity among our 5 health systems. At Henry Ford Health System, Kaiser Permanente Hawaii, Kaiser Permanente Colorado (KPCO), University of Pennsylvania (UPenn), and Marshfield Clinic Health System, there is a total of 1,603,546 patients 
eligible for the study. At KPCO, 99.6\% of adults in the health system live in metropolitan areas, whereas $87.6 \%$ of adults in Marshfield's health system live in micropolitan or rural areas. Within the UPenn health system, $34.0 \%$ of households are below the federal poverty limit (FPL) while only $0.7 \%$ of households within KPCO are below the FPL. That said, there are more accredited lung cancer screening facilities in the city of Philadelphia (828) than in the whole state of Colorado (623) - nearly 6 per square mile covering 1.58 million people vs 6 per 1000 square miles covering 5.61 million people.

Conclusion: Although we will still use proxies for measuring social determinants of health, by using multiple and more granular sources of geospatial data than census tract alone, we will be able to improve these proxies and, thus, better assess health disparities within our lung cancer screening study population.

\section{CHRONIC CONDITIONS, MULTIMORBIDITY, AND AGING POPULATIONS}

\section{OA8.01

\begin{abstract}
Multimorbidity in Chronic Obstructive Pulmonary Disease Across Three Geographic Regions of a Large Integrated Health Care Organization
\end{abstract}

Richard Mularski, ${ }^{1}$ Earnest Shen, ${ }^{2}$ Janet Lee, ${ }^{2}$ Phillip Crawford, ${ }^{1}$ Alan Go, ${ }^{3}$ Huong Nguyen ${ }^{2}$

${ }^{1}$ Kaiser Permanente Northwest, Portland, OR; ${ }^{2}$ Kaiser Permanente Southern California, Pasadena, CA; ${ }^{3}$ Kaiser Permanente Northern California, Oakland, CA

Background: Previous studies in limited geographic datasets have reported that more than $50 \%$ of chronic obstructive pulmonary disease (COPD) patients have at least 4 or more comorbidities. We aimed to identify comorbidity profiles in a community-based sample of patients with COPD from a large integrated health care system across 3 U.S. geographic regions. Methods: In this retrospective cohort study, we used latent class analysis (LCA) to identify comorbidity profiles in a population-based sample of 91,453 patients with a COPD diagnosis between 2011 and 2015. We included specific comorbid conditions from the Charlson comorbidity index (CCI) and accounted for variation in underlying prevalence of different comorbidities across study sites. Sociodemographic, clinical, and health care utilization data were obtained from electronic records.

Results: The mean age was 71 years, 55\% were females, 23\% were nonwhite, and $80 \%$ were former or current smokers. LCA identified 4 distinct comorbidity profiles with progressively higher CCI scores: low morbidity $(61 \%, 1.9 \pm 1.4)$, metabolicrenal $(21 \%, 4.7 \pm 1.8)$, cardiovascular $(12 \%, 4.6 \pm 1.9)$, and multimorbidity $(7 \%, 7.5 \pm 1.7)$. Patients with a low morbidity profile tended to be younger (68 vs 74 years, $\mathrm{P}<0.001$ ), were current smokers ( $20 \%$ vs $13 \%, \mathrm{P}<0.001)$, and more physically active $(51 \%$ engaged in at least some moderate activity in a week vs $40 \%, \mathrm{P}<0.001$ ) compared to patients in the higher morbidity profiles. FEV1 percentage of predicted, which was obtained from a subsample of patients, was not different across the morbidity profiles $(68 \% \pm 21 \%$ for low morbidity to $64 \%$ $\pm 19 \%$ for multimorbidity). There was a trend in increasing use of acute care (hospitalizations, observational stays, and emergency department visits) with increasing class-average CCI $(\mathrm{P}<0.001)$ in the year of cohort identification.

Conclusion: Our finding of 4 comorbidity clusters in COPD patients resonates with previous studies; previous psychological profile was not seen as CCI does not include mental health diagnoses. The distribution across clusters suggests that earlier studies using limited samples may overestimate the burden of comorbidities in community settings. We demonstrated independence of the multimorbidity profiles to airflow obstruction, though spirometry data was limited to half of the sample. Subsequent work will examine downstream health care utilization patterns across these 4 comorbidity profiles.

\section{OA8.02}

\section{A Multimodal Intervention for Improving Osteoporosis Treatment Initiation and Adherence}

Ryan Outman, ${ }^{1}$ Paul Muntner, ${ }^{1}$ Andrew Bradlyn, ${ }^{2}$ Lee Cromwell, ${ }^{2}$ Brandi Robinson, ${ }^{2}$ Douglas Roblin, ${ }^{3}$ Dennis Tolsma, ${ }^{2}$ Kenneth Saag ${ }^{1}$

${ }^{1}$ University of Alabama at Birmingham, Birmingham, AL; ${ }^{2}$ Center for Research and Evaluation, Kaiser Permanente Georgia, Atlanta, GA; ${ }^{3}$ Mid-Atlantic Permanente Research Institute, Rockville, $M D$

Background: Adherence to a prescribed bisphosphonate regimen is low, despite its demonstrated efficacy in reducing risk of osteoporotic fractures. The objective of this pilot randomized trial was to evaluate the potential for simple, short web-based videos to improve bisphosphonate initiation and adherence.

Methods: We recruited Kaiser Permanente Georgia members who were English speakers, 55 years of age or older, had internet access, and had an initial bisphosphonate prescription in the 30 days prior to invitation. Participants were randomized to a nurse phone consultation plus a web-based video ("enhanced intervention") or a webbased video alone ("basic intervention"). We also included nonparticipants ("passive refusals") in comparisons. The enhanced intervention included a video describing benefits of osteoporosis treatment along with several short videos of patient experience. Two weeks following the video, participants were asked to complete a nurse consultation by telephone. The nurse consultation focused on possible barriers to initiation and continuation of therapy. The basic intervention included a video that presented narrated bullet 
points of the benefits of osteoporosis treatment.

Results: The mean age for the enhanced intervention, basic intervention, and passive refusals was 69.3, 69.6, and 69.3 years, respectively; $19.1 \%, 32.1 \%$, and $28.2 \%$, respectively, were of black race. In the 180 days following the initial bisphosphonate order date, $90.5 \%$ of enhanced intervention participants, $85.7 \%$ of basic intervention participants, and $90.9 \%$ of passive refusals had at least one bisphosphonate fill ( $\mathrm{P}>0.4$ for all pairwise comparisons). Among participants with at least one bisphosphonate fill, $71 \%$ in the enhanced intervention, $68 \%$ of participants in the basic intervention, and only $37 \%$ of passive refusals had 180 days of bisphosphonate supply. The difference in days supply between the enhanced and basic interventions was not statistically significant; however, days supply in each of these two groups was greater than that among passive refusals $(\mathrm{P}<0.01)$.

Conclusion: In this sample of postmenopausal women, primary adherence to an initial prescription of a bisphosphonate was high; neither the enhanced nor basic intervention increased this percentage. The active intervention - whether basic or enhanced - improved refill adherence in the subsequent 180 days compared to matched postmenopausal women who did not participate in the trial.

\section{Abstract OA8.03 withdrawn by authors after initial publication}

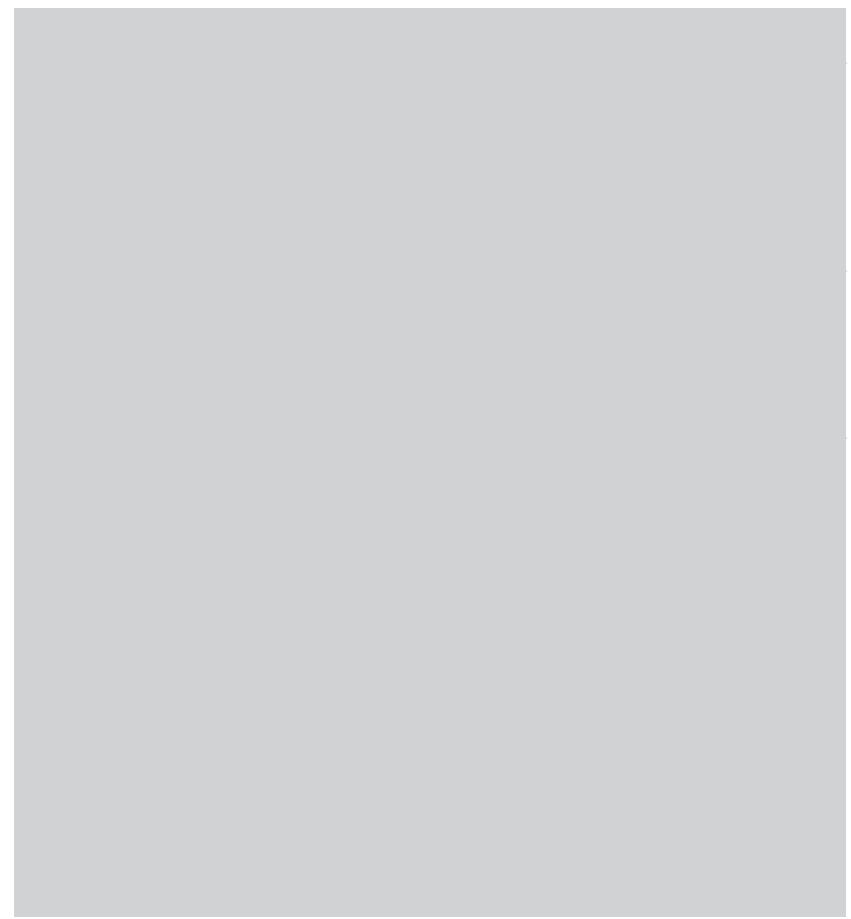

OA8.04

Early Weight Change as a Predictor of Long-Term Treatment Response in a Behavioral Lifestyle Program for Diabetes Prevention in Clinical Practice

Robert Romanelli, ${ }^{1}$ Qiwen Huang, ${ }^{1}$ Sylvia Sudat, ${ }^{2}$ Alice Pressman, ${ }^{2}$ Kristen Azar ${ }^{2}$

${ }^{1}$ Palo Alto Medical Foundation Research Institute, Sutter Health, Palo Alto, CA; ${ }^{2}$ Division of Research, Development \& Dissemination, Sutter Health, Walnut Creek, CA

Background: Evidence-based behavioral interventions for diabetes prevention have demonstrated efficacy in reducing diabetes risk, primarily though weight loss. Variation in treatment response is high yet is poorly understood when such programs are implemented within real-world clinical settings. The ability to identify potential nonresponders early during a behavioral lifestyle program (BLP) presents an opportunity to assess if more intensive treatment is needed. We sought to develop and validate a predictive model for the early identification of nonresponders to a 12-month BLP. Methods: Data were derived from the electronic health records of a Northern California health care delivery system. We included BLP participants $\geq 18$ years of age with 4 or more weight measurements recorded during the first 12 weeks of the program and a weight measurement at 12-month follow-up. We defined nonresponse as weight gain or no weight loss at 12 months from program initiation. We used logistic regression with percent weight change at 12 weeks from program initiation as the sole independent variable to predict nonresponse at 12 months. A secondary model included patient characteristics as independent 
variables. We performed 10-fold cross-validation for model assessment. We examined model performance by area under the receiver operating characteristic curve (AUROC), sensitivity, specificity, and positive (PPV) and negative (NPV) predictive values.

Results: The study cohort included 961 BLP participants; $30 \%$ were classified as nonresponders. For the model with percent weight change as the sole predictor, the AUROC was 0.785 , corresponding to fair discrimination of nonresponders from responders. The addition of patient characteristics resulted in slightly poorer discrimination (AUROC: 0.755). For the simpler model, sensitivity and PPV were maximized at 0.56 , with a specificity and NPV of 0.81 . With this model, BLP participants with a predicted probability of nonresponse of $\geq 41 \%$, corresponding to $<1.83 \%$ weight loss at 12 weeks from baseline, would be flagged as potential nonresponders and candidates for treatment intensification assessment.

Conclusion: In this cohort of BLP participants in a realworld clinical setting, percent weight change in the first 12 weeks, alone, predicts nonresponse at 12 months. Clinicians can apply this algorithm to identify and assess patients in potential need of treatment intensification, thereby improving long-term treatment response through an individualized, patient-centered approach to diabetes prevention.

\section{P5.01 \\ Personalized and Marginal Concurrent Risk in Older Persons With Multiple Chronic Conditions Receiving Polypharmacy}

\section{Heather Allore, ${ }^{1}$ Terrence Murphy, ${ }^{1}$ George Agogo, ${ }^{1}$ Gail McAvay ${ }^{1}$}

${ }^{1}$ Yale University, New Haven, CT

Background: Older adults accumulate chronic conditions requiring medications that bear on patient-centered outcomes. There is a need to understand how specific factors affect the probability of patient-centered outcomes, such as limitations in social activity and mobility that are correlated and nonmutually exclusive. Knowing the probability of experiencing pairs of patient-centered outcomes within the next year may help patients and providers achieve goals of care. We define and illustrate personalized concurrent risk (PCR), ie, the person-specific risk of an outcome in an interval of time while at concurrent risk for a separate outcome, and the corresponding marginal concurrent risk (MCR).

Methods: Data are from older community-dwelling persons with both hypertension and chronic obstructive pulmonary disease. Exposure was polypharmacy, defined as the use of 5 or more classes of prescription medications. The two concurrent patient-centered outcomes were social activity and mobility limitations. To address imbalances, propensity scores of each person's likelihood of polypharmacy were used to generate inverse probability of polypharmacy weights. These weights were applied to the models of the occurrence of limitations in social activity and mobility over two consecutive 1-year periods joined through a shared person-specific random intercept. PCRs and MCRs were calculated from weighted joint models of the two outcomes regressed on polypharmacy, age, sex, and arthritis.

Results: Among persons with polypharmacy, the MCRs for limitations in social activity and mobility were from $1.6 \%$ to $10.3 \%$ and from $10.1 \%$ to $86.8 \%$, respectively, dependent on the patient characteristics. However, the PCRs for limitations in social activity and mobility ranged from $0.1 \%$ to $97.7 \%$ and from $2.0 \%$ to $99.9 \%$, respectively, showing that information captured by jointly modeling through a shared person-specific random intercept increases the accuracy of the estimated probability of an outcome occurrence within a time frame.

Conclusion: PCR provides a personalized estimate of risk conditional on a patient's characteristics and exhibit heterogeneity. In contrast, MCR provides the average risk. Application of PCR and MCR to health care system datasets may facilitate personalized medicine and decisionmaking for patients that may face tradeoffs or value multiple outcomes by providing the risk of an outcome in the presence of another correlated outcome. SAS code is available.

P5.02

Recruitment Challenges in Trials of Low-Risk Health Care Delivery System Interventions: Improving Medication Safety Postdischarge in Older Adults

Kathryn Anzuoni, ${ }^{1}$ Kathleen M. Mazor, ${ }^{1}$ Terry Field, ${ }^{1}$ Alok Kapoor, ${ }^{2}$ Lawrence Garber, ${ }^{3}$ Jerry Gurwitz ${ }^{1}$

${ }^{1}$ Meyers Primary Care Institute, Worcester, MA; ${ }^{2}$ University of Massachusetts Medical School, Worcester, MA; ${ }^{3}$ Reliant Medical Group, Worcester, MA

Background: Recently hospitalized older adults are at increased risk for adverse drug events (ADEs) during the days immediately after discharge. We sought to test the effectiveness of a pharmacist-based intervention targeting these patients; the intervention included a home visit from a clinical pharmacist with medication review, patient education, and telephone follow-up. The challenges we encountered in enrolling older patients immediately posthospitalization led us to examine whether enrolled patients were representative of all invited eligible patients and to explore reasons for declining.

Methods: Adults who were $>50$ years of age, recently discharged from a local hospital, and prescribed at least one high-risk medication (ie, anticoagulants, diabetes agents, or opioids) were eligible to enroll. Eligible patients were contacted via telephone within 3 days of discharge and invited to participate; those who declined were asked their reason(s). We compared demographic and clinical characteristics of enrolled patients to characteristics of all invited eligible patients. 
Results: Overall, 9.9\% ( $\mathrm{n}=353)$ of 3563 eligible patients reached by phone enrolled in the trial. Enrolled participants tended to be younger than the population of reached eligible patients; $27 \%$ of reached eligible patients were 80 years or older compared to only $14 \%$ of enrolled patients. Enrolled patients were less likely to have been admitted through the emergency room than reached eligible patients ( $59 \%$ vs $69 \%$, respectively) and more likely to be on more than 1 high-risk medication compared to reached eligible patients ( $54 \%$ vs $48 \%$, respectively). Patients' reasons for declining suggested they did not perceive a need for help with medications: $15 \%$ did not expect the study would be useful; $10 \%$ believed they had all the medication information they needed; and 19\% relied on assistance from the Visiting Nurse Association, a spouse, or family member. Some patients $(6 \%)$ were too ill or too tired to enroll.

Conclusion: Recruiting older, recently hospitalized patients during the immediate posthospitalization period when patients are most at risk for ADEs resulted in a low enrollment rate, a loss of power, and an inability to look at subgroups that may be of particular risk. Better strategies are needed to encourage and facilitate participation of high-risk, vulnerable patients in low-risk health care system-based interventions.

\section{P5.03}

Kidney Graft Survival Outcomes for Older Adults Over Time: An Examination of Factors From the United Network for Organ Sharing Database

Cinamon Romers, ${ }^{1}$ Kiumars Zolfaghari, ${ }^{1}$ Jinmyoung Cho, ${ }^{1}$ Hilary Linderman, ${ }^{2}$ Laurel Copeland ${ }^{3}$

${ }^{1}$ Baylor Scott \& White, Temple, TX; ${ }^{2}$ Rutland Regional Medical Center, Rutland, VT; ${ }^{3} V A$ Central Western Massachusetts Healthcare System, Leeds, MA

Background: Kidney transplantation is a preferred treatment for individuals with end-stage renal disease. With an increase in life expectancy, there is a need for transplantation for aging patients. Due to comorbid health problems and increased morbidity, advanced age has been considered a contraindication for transplant. This study aims to explore factors associated with mortality and graft failure among patients 50 to 79 years old.

Methods: Data from the United Network for Organ Sharing registry identified 86,696 kidney transplant recipients from 2006 to 2015. Recipients were stratified into 3 age groups (50-59, 60-69, and 70-79 years). Graft mortality and patient mortality were examined for recipients at 30 days, 90 days, 1 year, 3 years, and 5 years posttransplant. Chi-squared test and Student's t-test were used to assess recipient's characteristics, and Kaplan-Meier survival curves evaluated unadjusted all-cause mortality by age groups. In addition, Cox proportional hazards regression models were applied to adjust for potential confounding variables in the posttransplantation survival analysis.

Results: Transplantation increased among the oldest group in comparison to the youngest group over the years $(\mathrm{P}<0.0001)$. Death not associated with graft failure was more common in the 70-79-year age group (22.1\%) than in the 60-69-year age group (15.4\%) and the 50-59year age group $(9.9 \%)$. When controlling for all-cause mortality associated variables (body mass index, diabetes, hypertension, peripheral vascular disease, dialysis at time of transplant, prior transplant experience, receiving multiple organs, and lengthy wait-list time), the 50-59 age group was $14 \%$ more likely to experience graft failure than was the 70-79 age group.

Conclusion: Age is a primary criterion in transplant eligibility. In our analyses, fewer older recipients experienced graft failure than younger recipients. Candidates in the youngest age group were most likely to receive multiorgan transplants, with worse outcomes, than candidates in the oldest age group. Careful consideration should be given to age as a primary allocation criterion factor.

\section{P5.04 \\ A Qualitative Examination of Experiences Managing Type 1 Diabetes Among Patients 18 to 26 Years of Age}

Tina Davis, ${ }^{1}$ Josh Barzilay, ${ }^{1}$ Doraina Walker-Williams, ${ }^{1}$ Brandi Robinson, ${ }^{1}$ Negah Rassouli ${ }^{1}$

\section{${ }^{1}$ Kaiser Permanente Georgia, Atlanta, GA}

Background: Sustaining target A1c levels early during diabetes is essential to preventing and delaying disease complications, including hypoglycemia and cardiovascular disease. Research suggests most adults in their 20s will not achieve target A1c until age 30. Longitudinal research indicates adolescents and young adults (AYA) have declining glycemic control during adolescence and higher rates of diabetes complications compared to adults with diabetes. Adolescents must manage developmental challenges, including transitioning into adulthood, cognitive development, and navigating relationships, while concurrently managing their chronic condition. Therefore, the purpose of this research study is to understand barriers and facilitators of type 1 diabetes management among AYA. Methods: We conducted one-on-one, qualitative phone interviews with Kaiser Permanente Georgia (KPGA) members $(\mathrm{N}=14)$ with type 1 diabetes (T1D) to understand their experiences with T1D management, treatment, and medication adherence. Eligibility criteria included age 18-26 years, a confirmed T1D diagnosis, current enrollment at KPGA, and English-speaking. The sample was dichotomized into two groups using their A1c levels in the past 2 years: "managers" (A1c level of $<7 \%$ ) and "non-managers" (A1c of $>9.5 \%$ ) were asked to participate. Interviews were audio recorded, transcribed verbatim, and 
cleaned. All study procedures were approved by the KPGA institutional review board. Two coders will use open coding to develop a codebook and axial coding to code transcripts using NVivo 12.0 software.

Results: Preliminary results are as follows. All participants reported challenges monitoring glucose levels, feeling isolated, finding jobs to accommodate health needs, transitioning between health care practices, financing the cost of care, and communicating the nuances of T1D to peers, family, intimate partners, and supervisors. "Managers" reported receiving support and education early in their diagnosis, including parental support, clinician support, and attending camps for children with T1D.

Conclusion: AYA need resources and education that concurrently address social, nonmedical needs related to learning to communicate T1D needs to others, navigating social experiences and T1D management, and education on navigating resources. While classes are available at KPGA, most focus on type 2 diabetes management. Group sessions tailored to needs of members with T1D are essential, and considering the widespread use of technology among AYA, there should be a mobile health component.

\section{P5.05}

Association Between Metabolic Syndrome and Recurrence in Older Adults Treated for NonMuscle-Invasive Bladder Cancer

Tullika Garg, ${ }^{1}$ Amanda Young, ${ }^{1}$ Maureen O'KeeffeRosetti, ${ }^{2}$ Terrence Murphy, ${ }^{3}$ Carmit McMullen, ${ }^{2}$ Matthew Nielsen, ${ }^{4}$ H. Lester Kirchner ${ }^{1}$

${ }^{1}$ Geisinger Health, Danville, PA; ${ }^{2}$ Kaiser Permanente Northwest, Portland, OR; ${ }^{3}$ Yale University School of Medicine, New Haven, CT; ${ }^{4}$ The University of North Carolina at Chapel Hill, Chapel Hill, NC

Background: Non-muscle-invasive bladder cancer (NMIBC) disproportionately affects older adults (median age: 73 years) who also have a high prevalence of chronic conditions, including metabolic syndrome (MetS). Prior research suggests that MetS is a risk factor for the development of bladder cancer; however, limited data exist on how MetS impacts oncologic outcomes. The objective was to evaluate the association between MetS and recurrence in older adults treated for NMIBC.

Methods: We identified 1485 older ( $\geq 60$ years) NMIBC patients (stage $\leq$ I) from two community-based health systems who underwent cancer treatment (bladder instillation and/or transurethral resection). MetS was defined as the presence of 3 of the following based on diagnosis codes or electronic health data: hypertension, hyperlipidemia, diabetes, or body mass index of $>30$. Follow-up time was determined by date of last follow-up in the cancer registry, and data was truncated at 10 years. Cox proportional hazards regression adjusting for competing risks was performed, adjusting for age, sex, smoking status, health system, and NMIBC stage/grade to model the association between MetS and time to recurrence.

Results: Overall, 423 patients (28.5\%) had MetS and 1062 patients did not $(71.5 \%)$. Median follow-up for the cohort was 5.9 years. Follow-up for patients with MetS was slightly shorter as compared to those without MetS (4.7 vs 6.4 years). Patients had a median of 1 recurrence (interquartile range: 1-2) over the study period. Individuals with MetS tended to be younger (mean age: 73 years vs 73.8 years), more frequently male ( $83.7 \%$ vs $76.8 \%$ ), and never smokers (81.6\% vs $71.4 \%)$. After adjusting for age, sex, smoking status, health system, and stage/grade, MetS was associated with a reduction in the risk of recurrence (adjusted hazard ratio: $0.77,95 \% \mathrm{CI}$ : $0.63-0.95, \mathrm{P}=0.01$ ).

Conclusion: In this cohort of older NMIBC patients from two health systems, we found that the presence of MetS was associated with a decreased risk of NMIBC recurrence. Further research is needed to understand the complex pathophysiology underlying NMIBC outcomes and metabolic syndrome. In future analyses, we will assess how factors such as lipid- or glucose-lowering medications influence this association.

P5.06

Prevalence of Social/Economic Needs Among Seniors in a Non-Safety-Net Health Plan

Nancy P. Gordon, ${ }^{1}$ Gabriel Escobar, ${ }^{1}$ Patricia Kipnis ${ }^{1,2}$

${ }^{1}$ Division of Research, Kaiser Permanente Northern California, Oakland, CA; ${ }^{2}$ Department of Healthcare Quality and Affordability Analytics, Kaiser Permanente Northern California, Oakland, CA

Background: Many health plans are looking at screening Medicare-age adults for social/economic risks (SERs). This study examined prevalence of SERs among seniors in a nonsafety-net health plan population.

Methods: In 2018, we conducted a mailed survey of a stratified (age, sex, COPS2 comorbidity score), random sample of English-speaking Kaiser Permanente (KP) Northern California members 65-89 years of age using KP's Your Current Life Situation questionnaire $(56 \%$ response, 2061 of 3065). Using responses from multiple questions, we classified adults as being at-risk for food insecurity (FoodInsec), housing insecurity (HousInsec), transportation insecurity (TransportInsec), financial strain (FinanStrain, defined as trouble paying for food, housing, utilities, medical needs, transportation, debts, other basics), social isolation (SocIsolRisk, defined as lives alone and not in a relationship), feeling overwhelmed by difficulties (Overwhelm), medical SERs (MedSER, defined as trouble paying for medical needs or medical-related transportation issues), and nonmedical SERs (NonMedSER, defined as 1 or more nonmedical SER, excluding SocIsolRisk). We estimated population prevalence of SERs using weighted 
survey data and evaluated differential risk for SERs by race/ethnicity, age group, sex, and COPS2 level ( $<45$ vs $\geq 45$ ) using multivariable logistic regression models that additionally controlled for being married/partnered and living in a residential facility.

Results: Overall prevalence of SERs was $8.5 \%$ FoodInsec, 8.4\% HousInsec, $4.3 \%$ TransportInsec, $12.0 \%$ FinanStrain, and $20.4 \%$ SocIsolRisk. Nearly $16 \%$ felt overwhelmed at least sometimes, $2 \%$ often. Approximately $5 \%$ had 1 or more MedSER and $22.5 \% 1$ or more NonMedSER (36\% if SocIsolRisk included). Logistic regression models showed that blacks and Filipinos were more than 2 times as likely as whites to be at risk for FoodInsec, HousInsec, TransportInsec, FinanStrain, Overwhelm, MedSER, and NonMedSER. Women were twice as likely as men to have SocIsolRisk but were not at greater risk for other SERs. A COPS2 level of $\geq 45$ more than doubled risk for all SERs, MedSER, and NonMedSER, while being married halved risk. SER risk was not associated with age.

Conclusion: Among seniors in this non-safety-net health plan, $5 \%$ of seniors had medical-related social/economic risks, while 3 times that percentage had 1 or more nonmedical-related risks that potentially could affect health and quality of life. Black or Filipino race and sicker seniors were more likely to have both medical and nonmedical social/economic risks.

\section{P5.07}

\section{Primary Care Treatment for Migraine: Experience} From a Large Health Care System

Alice Jacobson, ${ }^{1}$ Shruti Vaidya, ${ }^{1}$ Reeti Sharma, ${ }^{1}$ Victoria Chia, ${ }^{2}$ Dawn Buse, ${ }^{3}$ Richard Lipton, ${ }^{3,4}$ Walter Stewart, ${ }^{5}$ Alice Pressman ${ }^{1}$

${ }^{I}$ Sutter Health, Walnut Creek, CA; ${ }^{2}$ Amgen Inc, Thousand Oaks, CA; ${ }^{3}$ Albert Einstein College of Medicine, New York, NY; ${ }^{4}$ Montefiore Medical Center, New York, NY; ${ }^{5} H I N T$ Consulting, Orinda, CA

Background: The majority of those diagnosed with migraine seek treatment only from primary care, but little is known about the approach by which primary care providers (PCPs) manage migraine. We sought to compare prescribing patterns for migraine patients in a large health care system, comparing those who seek care from neurology (N-M) with those treated exclusively by a PCP (PC-M).

Methods: From electronic health records (EHR), we applied a previously validated migraine probability algorithm (MPA) to identify 94,149 PC-M and N-M patients at Sutter Health in Northern California from January 1, 2013, to December 31, 2017. We extracted medication orders for acute treatments (non-narcotic-analgesic, narcotic-analgesic, triptan, and other migraine-specific) and the most commonly-prescribed preventive treatments (beta-blocker, calcium channel blocker, antidepressant, anticonvulsant, and neurotoxin).
Because higher scores on the migraine probability algorithm indicate more extensive utilization, we classified $45 \%$ of PC-M and $55 \%$ of N-M patients with a maximum score as "frequent-consulters."

Results: During the 5-year period, PC-M patients $(\mathrm{n}=72,624,77 \%)$ were less likely to receive 1 or more acute $(81 \%$ vs $89 \%)$ or 1 or more preventive (55\% vs $81 \%$ ) prescription orders than N-M patients. Among frequentconsulters, a larger proportion of PC-M patients received orders for 1 or more acute treatments ( $98 \%$ vs $93 \%$ ) and the difference between PC-M and N-M in orders for 1 or more preventives was smaller ( $73 \%$ PC-M vs $86 \% \mathrm{~N}-\mathrm{M})$. The most commonly prescribed preventive type for PC-M and $\mathrm{N}-\mathrm{M}$ was antidepressant (41\% PC-M, 62\% N-M); among frequent-consulters, neurotoxin was the least prescribed $(<1 \%$ PC-M vs $9 \%$ N-M).

Conclusion: Neurology patients at Sutter Health were more likely to receive both acute and preventive medication orders than PC-M patients, with triptans and antidepressants most commonly ordered preventive and acute, respectively. However, among frequent-consulters, this relationship was reversed for acute treatments and attenuated for preventive. A limitation of this study is that using EHR data alone, we cannot reliably differentiate between chronic and episodic migraine. It is likely that neurologists treat more patients with chronic migraine than PCPs do, and that may account for the prescribing differences. Further study is underway to assess the impact of migraine severity on prescribing patterns using a patient survey combined with EHR.

P5.08

\section{Understanding Communications Challenges Between Care Facilities and Primary Care Physicians to Improve Care for Facility Residents}

\section{Elizabeth Liles, ${ }^{1}$ Paula Carder, ${ }^{2}$ Inga Gruß ${ }^{1}$ \\ ${ }^{1}$ Kaiser Permanente Center for Health Research, Portland, OR; ${ }^{2}$ Portland State University, Portland, OR}

Background: Assisted living includes all group homes not licensed as nursing homes that provide personal care and can respond to unscheduled needs in a homelike environment. In 2014, 30,200 residential care/assisted living (RC/AL) facilities in the United States housed more than 1 million residents. More than half are 85 years or older, and 4 out of 10 have dementia. They commonly require assistance with bathing or toileting. These vulnerable adults require timely evaluation of acute symptoms and injuries. State regulations mandate that $\mathrm{RC} / \mathrm{AL}$ residents receive nursing oversight of medications and chronic health conditions, and evaluation of acute symptoms and injuries such as those due to falls. States also set regulations for physician responsibilities, although the duties of primary care providers are vaguely defined. Communication difficulties arise in a system in which care is dispersed between facility staff and primary care medical offices. 
Methods: We conducted 3 focus groups with 21 primary care physicians (PCPs) at 3 Kaiser Permanente medical offices in the greater Portland metro region. The goal was to learn about communication between RC/AL staff and PCPs, including barriers and facilitators. We also surveyed more than 300 such facilities in Oregon, asking about their perceived successes and barriers in communicating with primary care offices.

Results: We found a lack of standardization of communications, challenges to incorporating different modes of communication, and a lack of transparency about the perceived importance of communications. From 276 returned surveys, the top 3 concerns about communications with primary care medical offices were lack of timely response to fax or phone message, physicians not providing necessary information on orders, and PCPs' lack of understanding of facilities' regulatory requirements. About $63 \%$ of respondents remarked that replies to messages sent to primary care offices via fax or phone were frequently slow.

Conclusion: Stakeholder input on communications between PCP offices and residential care/assisted living facilities revealed communications gaps. We will use our findings to draft standardized communications forms between PCP offices and RC/AL facilities that can be used by any physician group or health system. Improving communications will improve health, well-being, and safety for patients living in these facilities.

\section{P5.09}

\section{Emergency Department Recurring Visits for Adult Patients With Asthma or Asthma Plus Chronic Obstructive Pulmonary Disease}

\section{Zijun Shen, ${ }^{1}$ Reeti Sharma, ${ }^{1}$ Shruti Vaidya, ${ }^{1}$ Maria Moreno, ${ }^{1}$ Kristen Azar, ${ }^{1}$ Stephen Lockhart, ${ }^{1}$ Alice Pressman ${ }^{1}$}

\section{${ }^{I}$ Sutter Health, Walnut Creek, CA}

Background: Breathing difficulty (BD) is one of the most common reasons for emergency department (ED) encounters. As part of an initiative to advance health equity, Sutter Health, a large not-for-profit health care system in Northern California, implemented an educational intervention program for African American adult ED patients with a chief complaint of BD and history of asthma. Early evaluation showed that enrolled asthma patients with a comorbidity of chronic obstructive pulmonary disease (COPD) were more likely to return to the ED after intervention than those with asthma alone. We studied these two subgroups of ED patients to identify their patterns of ED utilizations that could inform the development of interventions to reduce readmissions.

Methods: We extracted data from electronic health records of adult asthma patients with ED encounters for BD at 4 Sutter Health hospitals in 2 counties in Northern California. Patients were classified as $\mathrm{AC}$ for those with a discharge diagnosis or history of COPD (COPD, chronic bronchitis or emphysema) and SA for those without. We defined readmission as returning to the ED with similar chief complaints within 30 days. We compared demographics and ED utilization between patients with SA and $\mathrm{AC}$ by readmission status.

Results: Among 3625 patients who met criteria during 2017, 2364 (65\%) had SA and 1261 (35\%) had AC. Readmissions differed by group, with 321 (12\%) of SA and 295 (25\%) of $\mathrm{AC}$ returning to the $\mathrm{ED}$ within 30 days $(\mathrm{P}<0.01)$. For both types of asthma patients, those who readmitted were more likely to be African American than those who did not (49\% vs $45 \%$ for $\mathrm{SA}, \mathrm{P}=0.07 ; 54 \%$ vs $46 \%$ for $\mathrm{AC}, \mathrm{P}=0.01$ ). Those with $\mathrm{AC}$ who readmitted were significantly older $(61 \pm 14.3$ years) than those with $\mathrm{SA}(44 \pm 17.9$ years; $\mathrm{P}<0.01)$, while gender patterns were similar.

Conclusion: Patients with $\mathrm{AC}$ are more likely to have recurring ED visits than those with SA. Further, those with $\mathrm{AC}$ who readmit are more likely to be African American and older than their SA counterparts. An adaptive approach to designing intervention programs for these different subgroups should include more customized education for patients with comorbid COPD who also differ culturally.

P5.10

Impact of Cardiac- and Noncardiac-Related Conditions on Adverse Outcomes in Patients Hospitalized With Acute Myocardial Infarction

Mayra Tisminetzky, ${ }^{1}$ Jerry Gurwitz, ${ }^{1}$ Joel Gore,${ }^{2}$ Ruben Miozzo, ${ }^{3}$ Darleen Lessard, ${ }^{2}$ Jorge Yarzebski, ${ }^{2}$ Robert Goldberg $^{2}$

${ }^{1}$ Meyers Primary Care Institute, Worcester MA; ${ }^{2}$ University of Massachusetts Medical School, Worcester MA; ${ }^{3} J o h n s$ Hopkins University, Baltimore MD

Background: To examine the effects of cardiac- and noncardiac-related conditions on in-hospital complications and 7- and 30-day rehospitalizations after an acute myocardial infarction.

Methods: The study population consisted of 3863 patients, 65 years and older, hospitalized with acute myocardial infarction at 3 medical centers in Worcester, Massachusetts, during 6 annual periods between 2001 and 2011. Individuals were categorized into 4 groups based on the presence of cardiacand noncardiac-related conditions: those with $0-2$ cardiac and 0 noncardiac conditions; $0-2$ cardiac and 1 or more noncardiac conditions; 3 or more cardiac and 0 noncardiac conditions; and 3 or more cardiac and 1 or more noncardiac conditions.

Results: The median age of the study population was 79 years; $49 \%$ were men. Overall, $28 \%$ of patients had $0-2$ cardiac and 0 noncardiac conditions, $21 \%$ had $0-2$ cardiac and 1 or more noncardiac conditions, $20 \%$ had 3 or more cardiac and 0 noncardiac conditions, and $31 \%$ had 3 or more cardiac and 1 or more noncardiac conditions. After 
multivariable adjustment, individuals with 3 or more cardiac and 1 or more noncardiac conditions were at greatest risk for all adverse outcomes. Patients who presented with cardiacrelated conditions were at higher risk of dying or developing complications during hospitalization, whereas those with noncardiac-related conditions were at slightly higher risk of being rehospitalized within 7 days.

Conclusion: Patients hospitalized with acute myocardial infarction carry a significant burden of cardiac- and noncardiac-related conditions. Increased attention to the management of this vulnerable population is warranted.

\section{CARDIOVASCULAR DISEASE}

\section{OA12.01 \\ Long-Term Effects on Cardiovascular Events of Home Blood Pressure Telemonitoring With Pharmacist Management in Patients With Uncontrolled Hypertension}

Karen L. Margolis, ${ }^{1}$ Stephen Dehmer, ${ }^{1}$ Michael Maciosek, ${ }^{1}$ JoAnn Sperl-Hillen, ${ }^{1}$ Stephen Asche, ${ }^{1}$ Anna Bergdall, ${ }^{1}$ Beverly B. Green, ${ }^{2}$ Rachel Nyboer, ${ }^{1}$ Pamala A. Pawloski, ${ }^{1}$ Nicole Trower, ${ }^{1}$ Patrick J. O'Connor

${ }^{1}$ HealthPartners Institute, Minneapolis, MN; ${ }^{2}$ Kaiser Permanente Washington Health Research Institute, Seattle, WA

Background: Uncontrolled hypertension is a leading contributor to cardiovascular $(\mathrm{CV})$ disease. We previously reported results of a cluster-randomized trial evaluating a 1-year home blood pressure telemonitoring and pharmacist management intervention, with significant reductions in blood pressure favoring the intervention group for 24 months. This analysis examined the intervention effect on CV events over 5 years of follow-up.

Methods: The Hyperlink trial randomized 16 primary care clinics with 450 patients with uncontrolled hypertension to either telemonitoring intervention $(\mathrm{TI}, \mathrm{n}=228)$ or usual care (UC, n=222). CV events and deaths were ascertained though self-reports and electronic health records. Medical records for all potential events were adjudicated by trained reviewers blinded to intervention assignment. The primary composite endpoint included nonfatal myocardial infarction (MI), nonfatal stroke, hospitalized heart failure (HF), and $\mathrm{CV}$ death. Coronary revascularization was included in a secondary composite endpoint. Generalized estimating equations with robust standard errors were used to test differences in $\mathrm{CV}$ incidence by treatment group. Marginal Cox models were used to estimate the hazard ratio for time to first event by treatment group. Both methods account for the cluster-randomized design.

Results: There were $15 \mathrm{CV}$ events (5 MI, 4 stroke, $5 \mathrm{HF}, 1$ $\mathrm{CV}$ death) among 10 patients in the TI group and 26 events
(11 MI, 12 stroke, $3 \mathrm{HF}$ ) among 19 patients in the UC group. The incidence of the primary $\mathrm{CV}$ composite endpoint was $4.4 \%$ in the TI group vs $8.6 \%$ in the UC group (odds ratio: 0.49 , 95\% CI: $0.21-1.13, \mathrm{P}=0.09$ ). Including 2 coronary revascularizations in the TI group and 10 in the UC group, the incidence of the secondary $\mathrm{CV}$ composite endpoint was $5.3 \%$ in the TI group vs $10.4 \%$ in the UC group (odds ratio: $0.48,95 \% \mathrm{CI}: 0.22-1.08, \mathrm{P}=0.08)$. In the time-to-first-event analysis for the primary outcome, hazard ratio was 0.50 (95\% CI: $0.22-1.11, \mathrm{P}=0.09$ ). For the secondary endpoint including coronary revascularization, hazard ratio was 0.49 (95\% CI: 0.23-1.04, $\mathrm{P}=0.06$ ).

Conclusion: The intervention lowered blood pressure and may have reduced $\mathrm{CV}$ events by $50 \%$ over 5 years, even in this small study. Cost-effectiveness estimates using microsimulation models will be presented.

OA12.02

Blood Pressure Checks and Diagnosing
Hypertension (BP-CHECK): A Randomized
Controlled Diagnostic Study Comparing Clinic,
Home, and Kiosk to 24-Hour Ambulatory BP
Monitoring - Comparative Accuracy Primary
Outcome Results

Beverly B. Green, ${ }^{1}$ Melissa L. Anderson, ${ }^{1}$ Andrea J. Cook, ${ }^{1}$ Kelly J. Ehrlich, ${ }^{1}$ Yoshio N. Hall, ${ }^{2,4}$ Predjag Klasnja, ${ }^{1}$ Karen L. Margolis, ${ }^{3}$ Jennifer B. McClure, ${ }^{1}$ Matthew J. Thompson ${ }^{4}$

${ }^{1}$ Kaiser Permanente Washington Health Research Institute, Seattle, WA; ${ }^{2}$ Kidney Research Institute, Seattle, WA; ${ }^{3}$ HealthPartners Institute, Minneapolis, MN; ${ }^{4}$ Department of Family Medicine, University of Washington, Seattle, WA

Background: The U.S. Preventive Services Task Force recommends out-of-office blood pressure (BP) measurements before making a new diagnosis of hypertension, using 24-hour ambulatory monitoring (ABPM) or home BP monitoring. However, this approach is not common in routine practice. Blood Pressure Checks and Diagnosing Hypertension (BP-CHECK) is a randomized controlled diagnostic study comparing the accuracy and acceptability of clinic-, home-, and kiosk-based BP monitoring to ABPM for diagnosing hypertension.

Methods: Within an integrated health care system, adults 18-85 years of age without diagnosed hypertension, on no hypertension medication, and with elevated BPs in clinic and at the baseline research visit are randomized to 1 of 3 regimens for diagnosing hypertension: 1) clinic BPs, 2) home BPs, or 3) kiosk BPs. All participants subsequently complete ABPM. Clinic BP patients are asked to make a clinic appointment to have their BP rechecked. Home BP patients are trained to use a validated home BP monitor and asked to check their BP twice a day, with 2 measurements each time for 5 days. Kiosk BP patients are trained to use a validated BP kiosk and asked to check their BP at least 3 
more days with 3 measurements each time. All patients are asked to complete ABPM at 3 weeks. All BP data is being collected electronically (electronic health record, Bluetooth, web). The primary outcome is the accuracy of each method as compared to ABPM. Secondary outcomes include adherence to the assigned protocol and patient-reported outcomes related to diagnostic testing (discomfort, anxiety, perception of accuracy). Linear regression models will estimate the mean difference in systolic BP between the diagnostic and ABPM reference standard measures for each group.

Results: At the time of abstract submission 427 of 510 (84\%) of the expected total participants had been enrolled. Participants were $48 \%$ female, were $21 \%$ nonwhite or Hispanic, and had a mean body mass index of 30. Average baseline BP was 150 $\mathrm{mmHg}$ systolic and $88 \mathrm{mmHg}$ diastolic. Enrollment ends in January 2019, and we expect to have preliminary primary accuracy outcomes at the time of the meeting.

Conclusion: BP-CHECK will inform which hypertension diagnostic methods are most accurate, acceptable, and feasible to implement in primary care.

\section{OA12.03}

\section{Real-World Outcomes Associated With Idarucizumab: Population-Based Retrospective Cohort Study}

\author{
Sonal Singh, ${ }^{1}$ Amit Nautiyal, ${ }^{2}$ Kathy Belk ${ }^{3}$ \\ ${ }^{1}$ University of Massachusetts Medical School / Meyers \\ Primary Care Institute, Worcester, MA; ${ }^{2}$ Pulmonary and \\ Critical Care, Department of Medicine, Albany Medical \\ College, Albany, NY; ${ }^{3}$ Health Data Analytics, Irving, TX
}

Background: Idarucizumab reverses the anticoagulant effect of dabigatran, but few comparative studies have reported on clinical outcomes with idarucizumab. Our objective was to determine the effect of idarucizumab on clinical outcomes.

Methods: We conducted a retrospective cohort in a nationally representative sample of hospitals in the United States. The study population included adults $\geq 18$ years of age who were hospitalized for dabigatran-associated major bleeding between January 1, 2015, and December 31, 2017. We compared idarucizumab-exposed patients to the unexposed group. Our primary outcome of interest was in-hospital mortality.

Results: We included 266 exposed and 1345 nonexposed participants across 271 hospitals. Among participants with gastrointestinal bleeding, there was no statistically significant difference in the odds of in-hospital mortality $(9$ of 153 [5.9\%] vs 37 of 1124 [3.3\%]; adjusted odds ratio: 1.39 , 95\% CI: $0.51-3.45)$ between the idarucizumabexposed and nonexposed groups. Among participants with intracranial bleeding, there was an excess of in-hospital mortality (13 of 112 [11.6\%] vs 6 of 217 [(2.8\%]) associated with idarucizumab exposure, but limitations include sparse data and the inability to rule out residual confounding or confounding by disease severity.
Conclusion: Among a large nationally representative sample of adult patients with dabigatran-associated intracranial bleeding in the United States, we found no difference in in-hospital mortality among patients with gastrointestinal bleeding associated with idarucizumab exposure. An excess risk of in-hospital mortality associated with idarucizumab exposure among participants with intracranial bleeding deserves further exploration.

\section{OA12.04}

Impact of a Multicomponent Care Transition Intervention on Hospitalizations and Emergency Department Visits in Patients Diagnosed With Venous Thromboembolism

\section{Alok Kapoor, ${ }^{1}$ David McManus, ${ }^{1}$ Bruce Barton, ${ }^{2}$ Chad Darling, ${ }^{2}$ Saud Javed, ${ }^{2}$ Kathleen M. Mazor ${ }^{1}$}

${ }^{1}$ University of Massachusetts Medical School / Meyers Primary Care Institute, Worcester, MA; ${ }^{2}$ University of Massachusetts Medical School, Worcester, MA

Background: Hospitalizations and emergency department (ED) visits after venous thromboembolism (VTE) diagnosis are the cause of significant health burden in the United States, yet few interventions have specifically targeted these outcomes for patients with VTE. We assessed the impact of a multicomponent intervention on hospitalizations and ED visits in the 90 days after VTE diagnosis.

Methods: We randomized patients adults with new episode of VTE to usual care or a multicomponent intervention consisting of home pharmacist visit in the week after diagnosis, illustrated medication instructions, and follow-up phone call with an anticoagulation expert scheduled for 8 to 30 days from time of diagnosis. To examine the impact of the intervention we measured the incidence of hospitalizations and ED visits and identified those visits related to the index condition (ie, VTE, bleeding, or anticoagulation) and the preventability of these visits. To assess the relative effectiveness of the intervention we calculated an incidence rate ratio of the count of hospitalizations and ED visits, adjusting for potential confounders from residual imbalance of demographic and clinical variables.

Results: We enrolled 77 and 85 patients in the intervention and control groups, respectively. The incidence rate was 4.50 vs 6.01 visits per 1000 patient days in the intervention vs control groups. Most of the events in the control group were not related to the index condition $(21 \%)$, and of those that were related to it, most were not preventable $(25 \%)$. The adjusted incidence rate ratio (IRR) for the intervention was 1.05 (95\% CI: 0.57-1.91).

Conclusion: Patients have a significant number of hospital and ED visits in the 90 days after randomization, but most visits were not related to recurrent VTE, bleeding, or anticoagulation and were not preventable. After adjustment for confounders, there was no difference in utilization 
between intervention and control patients. Future research should focus on testing other interventions for patients with VTE or determining whether our intervention would be beneficial in other groups of patients.

\section{P2.01}

\section{Statin Therapy and Risk of Incident Diabetes Mellitus in Adults With Cardiovascular Risk Factors}

\author{
Kevin Kheder, ${ }^{1}$ Andrew Ambrosy, ${ }^{1}$ Dongjie Fan, ${ }^{2}$ Sue \\ Sung, ${ }^{2}$ Joan C. Lo, ${ }^{1}$ Alan $\mathrm{Go}^{2}$ \\ ${ }^{1}$ Kaiser Permanente Northern California, San Francisco, \\ CA; ${ }^{2}$ Division of Research, Kaiser Permanente Northern \\ California, Oakland, $C A$
}

Background: Several meta-analyses have found statin use to be associated with an increased risk of incident diabetes mellitus that is proportional to the intensity of lipid lowering therapy (LLT). However, the magnitude of this increased risk in nondiabetic adults with cardiovascular $(\mathrm{CV})$ risk factors has not been well-defined.

Methods: The Kaiser Permanente CHAMP study identified nondiabetic adults with $\mathrm{CV}$ risk factors and no prior LLT between January 1, 2008, and December 31, 2010. CV risk factors included known atherosclerotic $\mathrm{CV}$ disease (ASCVD), an elevated (>190 mg/dL) low-density lipoprotein cholesterol (LDL-C), or an LDL-C from 70 to $189 \mathrm{mg} / \mathrm{dL}$ and an estimated 10-year ASCVD risk $>7.5 \%$. Incident diabetes mellitus was defined as more than 2 abnormal tests (ie, hemoglobin A1c of $>6.5 \%$ or a fasting blood glucose of $>126 \mathrm{mg} / \mathrm{dL}$ ) or as 1 abnormal test and 1 new diagnostic code or medication for diabetes mellitus.

Results: There were 213,289 nondiabetic adults initially identified as having high CV risk. A total of 28,149 patients with a new statin prescription were matched to an equal number of patients with no prior LLT. Patients initiated on a statin were similar in terms of age $(67.9 \pm 9.4$ years $)$ and gender (42.8\% women) but were more likely to have LDL-C of $>130 \mathrm{mg} / \mathrm{dL}(71.1 \%$ vs $34.8 \%)$ or a prior myocardial infarction $(6.0 \%$ vs $0.1 \%)$. The unadjusted rate (per 100 person-years) of incident diabetes mellitus was low $(0.55$, 95\% CI: $0.52-0.59)$ but was marginally higher in patients who were treated with a statin $(0.69,95 \%$ CI: $0.64-0.74)$ vs no LLT (0.42, 95\% CI: 0.38-0.46). After adjustment, statin therapy was associated with an increased risk of incident diabetes mellitus (adjusted hazard ratio [aHR]: 1.17, 95\% CI: 1.02-1.34). Results were consistent in a sensitivity analysis comparing the low-potency statin group to no LLT (aHR: 1.22, 95\% CI: 1.06-1.40).

Conclusion: Among nondiabetic adults at high risk for ASCVD, initiation of LLT with a statin was independently associated with a modestly increased risk of incident diabetes mellitus.
P2.02

Optimization of a Risk Calculator to Predict LongTerm Outcomes and Utilization After Percutaneous Coronary Intervention

\author{
Samuel Savitz, ${ }^{1}$ Matthew Solomon, ${ }^{1}$ Sue Hee Sung, ${ }^{1}$ \\ Thomas Leong, ${ }^{1}$ Alan $\mathrm{Go}^{1}$
}

\section{${ }^{1}$ Kaiser Permanente Northern California, Oakland, CA}

Background: Risk calculators for clinical outcomes and utilization have become increasingly common tools to assist in cardiovascular care. We aimed to improve a risk calculator for predicting long-term outcomes and utilization after percutaneous coronary intervention (PCI). The strategies we evaluated for potentially improving the risk calculator included 1) adding predictors that have not typically been used in clinical risk calculators, and 2) using alternative machine learning approaches.

Methods: The original risk calculator used data from 2008 to 2012 for patients who received PCI at Kaiser Permanente Northern California, an integrated health care delivery system caring for $>4$ million members. The risk calculator predicted outcomes at 1 year for death, coronary heart disease hospitalization and emergency room visits, and repeat revascularization. The original model used Cox proportional hazards (Cox $\mathrm{PH})$ regression with backwards selection to estimate absolute risks. We evaluated the potential value of novel potential predictors in three domains: 1) area-based socioeconomic status; 2) access to care; and 3) patient behavior measures. We assessed for change in the C-index and the net reclassification index (NRI). The alternative modeling approaches were 1) Cox $\mathrm{PH}$ with lasso regression, 2) Cox $\mathrm{PH}$ with ridge regression, 3) Cox $\mathrm{PH}$ with elastic net regression, and 4) conditional inference trees. The improvement in predictive discrimination was assessed using changes in the C-index.

Results: The NRI results showed significant but small improvements from adding access to care measures but no improvement for the other domains. However, there was a consistent pattern across all three domains of only small, nonsignificant improvement in the $\mathrm{C}$-index after adding the novel predictors. The alternative methodological approaches also resulted in only small, nonsignificant improvement in the $\mathrm{C}$-index for any approach or outcome.

Conclusion: The new predictors and alternative approaches did not significantly improve the discrimination for risk predictions after PCI. Variables that measure socioeconomic status and access to care may be less important predictors in the context of integrated care structures. Improving the risk calculator may still be possible by identifying additional variables more related to patient clinical characteristics or implementing more advanced modeling approaches (eg, deep learning). 
P2.04

\section{Statin Discontinuation and Cholesterol Control in Central Wisconsin Adults With Cardiovascular Disease}

\section{Jeffrey VanWormer,${ }^{1}$ Sumithra Tirunagaram, ${ }^{1}$ Shereif Rezkalla $^{1}$}

\section{${ }^{1}$ Marshfield Clinic, Marshfield, WI}

Background: The revised clinical practice guidelines on the treatment of blood lipids included broad shifts in statin eligibility and targets of statin therapy. Some recent trial evidence has called into question the subduing of the previous treat-to-target emphasis on low-density lipoprotein (LDL) cholesterol. This study examined Wisconsin adults with cardiovascular disease (CVD) over 2 years since the new cholesterol guidelines were released. Specific aims were to 1) identify the proportion of patients who persisted with statin therapy, and 2) compare LDL cholesterol reduction between patients who persisted vs discontinued statin therapy.

Methods: This was a retrospective analysis of data from adults in the Marshfield Epidemiologic Study Area with a first CVD event between January 1, 2014, and December 31, 2015. Outcomes included statin discontinuation and LDL change over 1 year since their CVD event. Analyses were adjusted for age, gender, baseline LDL, and time to postLDL measurement.

Results: There were 461 patients who initiated (or continued with their existing) statin therapy following their CVD event. Of these, 27 (6\%) discontinued statin therapy within 1 year. The primary documented reasons for statin discontinuation were patient-reported side effects $(41 \%$; mainly myalgia) and beliefs that statin medication was unnecessary $(22 \%)$. No reason for statin discontinuation was documented for $30 \%$ of patients. Those who discontinued their statin had a median atorvastatin-equivalent dose of $10 \mathrm{mg}$, while statin persisters had a median dose of $20 \mathrm{mg}$. After 1 year, statin discontinuers had $86 \%$ lower adjusted odds of having LDL less than $70 \mathrm{mg} / \mathrm{dL}$, relative to statin persisters $(\mathrm{P}=0.009)$.

Conclusion: As expected, statin use was widespread among central Wisconsin adults with CVD. But some patients discontinued statin therapy and, although this had a usual negative impact on their LDL, it did not appear to be related to high-intensity statin prescriptions (which were uncommon). Reasons for statin discontinuation were not consistently documented in the medical record. Educational needs remain for clinicians to adhere to statin dosage guidelines and for some patients to adhere to their statin prescription. Future research should examine how lipids are managed over time among statin discontinuers as well as long-term impacts on recurrent CVD events.

\section{CANCER}

\section{OA13.01 \\ Financial Incentives to Increase Colorectal Cancer Screening Uptake and Decrease Screening Disparities: A 3-Arm Randomized Controlled Trial}

Beverly B. Green, ${ }^{1}$ Melissa L. Anderson, ${ }^{1}$ Andrea J. Cook, ${ }^{1}$ Jessica Chubak, ${ }^{1}$ Sharon Fuller, ${ }^{1}$ Kilian J. Kimbel, ${ }^{1}$ Jeffrey T. Kullgren, ${ }^{2}$ Richard T. Meenan, ${ }^{3}$ Sally W. Vernon ${ }^{4}$

${ }^{1}$ Kaiser Permanente Washington Health Research Institute, Seattle, WA; ${ }^{2}$ University of Michigan Medical School, Ann Arbor, MI; ${ }^{3}$ Kaiser Permanente Center for Health Research, Portland, OR; ${ }^{4}$ The University of Texas School of Public Health at Houston, Houston, TX

Background: Colorectal cancer (CRC) screening rates are suboptimal, particularly among disadvantaged populations. The study objective was to determine whether fixed (money) or probabilistic (lottery) financial incentives conditional on completion of CRC screening increased screening uptake and decreased screening disparities.

Methods: A parallel 3-arm randomized controlled trial was conducted between February 2017 and May 2018 with 22 medical centers in an integrated health care system in Washington State; 838 participants, age-eligible and overdue for CRC screening, were enrolled and randomized to either: 1) up to 3 mailings with information on the importance of CRC screening, screening test choices, a mailed fecal immunochemical test (FIT), and a reminder letter; or 2) this plus 1 of 2 different financial incentives - guaranteed money $(\$ 10)$ or probabilistic lottery (1 in 10 chances of $\$ 50)$ - conditional on completion of CRC screening. The primary outcome was completion of CRC screening within 6 months. Secondary outcomes included FIT completion and colonoscopy completion within 6 months. Intervention effects were compared across sociodemographic subgroups and self-reported psychosocial measures.

Results: Participants were predominantly female (65\%) and nonwhite or of Hispanic ethnicity (48\% nonwhite race, $12 \%$ Hispanic). Completion of any CRC screening was not significantly higher among + Monetary (76.6\%) and +Lottery $(74.7 \%)$ than mail-only $(71.5 \%)$ participants; $\mathrm{P}=0.11$. When limiting the analysis to FIT, there was a statistically significant intervention effect $(\mathrm{P}=0.032)$, with a net increase of $8.1 \%$ (+Monetary) and 7.0\% (+Lottery) compared to mail-only. Patient subgroups more responsive to financial incentives included those with Medicaid insurance (FIT completion rates: $42.9 \%$ mail-only, $81.8 \%$ +Monetary, $82.6 \%$ +Lottery; $\mathrm{P}=0.028)$ and those selfreporting opt-out behaviors (eg, not seeking health care unless ill) (FIT completion rates: 50.7\% mail-only, 68.7\% +Monetary, 67.7\% +Lottery; $\mathrm{P}=0.016$ ). 
Conclusion: Financial incentives increased FIT uptake but not colonoscopy completion. Financial incentives combined with direct mailing of FIT kits may decrease FIT completion disparities among some disadvantaged and harder-to-reach groups.

\section{OA13.02}

\section{Diagnosis and Treatment of Neuropathy Postchemotherapy in Three Health Systems}

\author{
Alyce Adams, ${ }^{1}$ Mary Becker, ${ }^{2}$ Jessica Chubak, ${ }^{3}$ Laura \\ Ichikawa, ${ }^{3}$ Tatjana Kolevska, ${ }^{4}$ Lin Ma, ${ }^{1}$ Pamala A. \\ Pawloski, ${ }^{2}$ Donna Rivera, ${ }^{5}$ Rebecca Ziebell ${ }^{3}$
}

${ }^{1}$ Division of Research, Kaiser Permanente Northern California, Oakland, CA; ${ }^{2}$ HealthPartners Institute, Minneapolis, MN; ${ }^{3}$ Kaiser Permanente Washington Health Research Institute, Seattle, WA; ${ }^{4}$ Oncology and Hematology, Kaiser Permanente Northern California, Vallejo, CA; ${ }^{5}$ National Cancer Institute, Bethesda, MD

Background: We examined factors associated with peripheral neuropathy (PN), a common side effect of chemotherapy, and PN treatment.

Methods: This observational study used data from Kaiser Permanente in Northern California and Washington and from HealthPartners in Minnesota. We included active ( $\geq 1$ in-person visit) enrolled members $\geq 40$ years of age with diagnosis of invasive cancer (January 1, 2010, to December $31,2014)$ and who initiated select chemotherapies (eg, platinums, taxanes) within 12 months of cancer diagnosis. We excluded patients previously diagnosed or treated for PN. Patients were followed until death, disenrollment, new cancer diagnosis, or study end (December 31, 2016). The primary outcomes were time to clinical diagnosis of PN from start of chemotherapy and time to treatment after PN diagnosis. We calculated cumulative incidence curves accounting for competing risks and used Cox proportional hazards models to identify potential risk factors.

Results: We identified 13,058 patients, 23.1\% (95\% CI: $22.4 \%-23.8 \%$ ) of whom received a PN diagnosis within 1 year of chemotherapy. Factors associated with higher rates of PN diagnosis included black race (hazard ratio: 1.23 [95\% CI: 1.10-1.38]), diabetes (1.14 [1.05-1.25]), chronic pain (1.25 [1.17-1.33]), nonchemotherapy medications associated with PN (1.15 [1.06-1.24]), comorbidity (1.19 [1.10-1.29]), and concurrent platinum and taxane use compared to platinum alone (1.29 [1.18-1.42]) or taxane alone (1.23 [1.12-1.34]). PN rates also varied by health system and age (lower rates at $40-49$ and $\geq 70$ years). One-third of patients with a PN diagnosis $(95 \%$ CI: $30.9 \%-33.9 \%)$ started new PN treatment within 1 year. Treatment rates were associated with black race (1.24 [1.03$1.50])$, chronic pain (1.26 [1.12-1.40]) and recency of cancer diagnosis. Rates also varied across health systems.

Conclusion: Type of chemotherapy, comorbidity, race, and ethnicity were associated with higher rates of PN. Yet, variations in PN treatment were not adequately explained by clinical risk factors. Additional studies are needed to begin understanding observed variation in PN diagnosis and treatment and to identify strategies for improving patient care and outcomes.

OA13.03

\section{A Cost-Effectiveness Analysis of PARP Inhibitors as Maintenance Therapy for Patients With Recurrent Ovarian Cancer}

\author{
Lixian Zhong, ${ }^{1}$ Anh Thu Tran, ${ }^{1}$ Taylor Tomasino, ${ }^{1}$ \\ Elizabeth Nugent, ${ }^{2}$ Judith Smith ${ }^{2}$
}

${ }^{1}$ Texas A\&M University, College Station, $T X ;{ }^{2}$ The University of Texas Health Science Center at Houston, Houston, TX

Background: Ovarian cancer is the most fatal gynecologic cancer in the United States. Recently, a new class of therapeutic agent, the poly (ADP-ribose) polymerase (PARP) inhibitor, has been approved by the Food and Drug Administration to be used in advanced ovarian cancer patients who have gone through platinum-based chemotherapy and relapsed. The aim of the study is to evaluate the cost-effectiveness of two PARP inhibitor therapies, olaparib and niraparib, as maintenance therapy for patients with platinum-sensitive, recurrent ovarian cancer.

Methods: We constructed a decision-tree model to evaluate the cost-effectiveness of olaparib and niraparib compared to placebo. The study was done from a U.S. health care sector perspective. Costs included drug costs and costs of disease monitoring and management of adverse events throughout the treatment course. Costs were estimated from the Redbook, Medicare reimbursement rate, and literature in 2017 U.S. dollars. Clinical effectiveness was measured in progressionfree survival based on clinical trial results (NCT00753545, NCT01874353, and NCT01847274). Cost-effectiveness was measured using the incremental cost-effectiveness ratio (ICER). Results: At base case, niraparib was the more effective treatment option, with slightly higher PFS, followed by olaparib. The ICERs for niraparib and olaparib compared to common baseline placebo were $\$ 235 \mathrm{~K}$ and $\$ 287 \mathrm{~K}$ per PFS life-year, respectively, with olaparib being extendeddominated by niraparib. Both drugs were associated with lower ICERs in patients with $g B R C A$ mutation(s) than in patients without $g B R C A$ mutation(s). One-way sensitivity analysis suggested that drug prices and PFS could impact ICERs significantly, but the ICERs remained above $\$ 100 \mathrm{~K} /$ PFS within the plausible ranges of all parameters. Probabilistic sensitivity analysis suggested that niraparib and olaparib were associated with higher net benefits only when willingness to pay (WTP) was above \$210K per PFS life-year thresholds.

Conclusion: PARP inhibitors will extend PFS in platinumsensitive, recurrent ovarian cancer patients but also are associated with high drug acquisition costs. The base case ICERs were around or above $\$ 250 \mathrm{~K} / \mathrm{PFS}$ in our model. No formal WTP threshold for health technology assessment 
exists in the United States. At a reference WTP of $\$ 100 \mathrm{~K}$ per PFS life-year, they may not be cost-effective options.

\section{OA13.04 \\ Understanding Referrals to Outpatient Palliative Care and Goals-of-Care Discussions With Individuals Diagnosed With Stage IV Advanced Cancer}

\author{
Ellis Dillon, ${ }^{1}$ Jinnan Li, ${ }^{1}$ Amy Meehan, ${ }^{1}$ Su-Ying Liang, ${ }^{1}$ \\ Steve Lai, ${ }^{1}$ Natalia Colocci, ${ }^{1}$ Harold S. Luft' \\ ${ }^{1}$ Palo Alto Medical Foundation, Palo Alto, CA
}

Background: For individuals diagnosed with a stage IV cancer, earlier referral to palliative care may improve patient outcomes. We know little about when, if, and how patients and their providers discuss palliative care.

Methods: At a multispecialty practice with an established outpatient palliative care program, we identified stage IV cancer patients diagnosed from January 1, 2012, to December 31, 2017. We analyzed electronic health record data of patients who had died regarding formal referrals to and encounters with palliative care. We also conducted interviews with 16 oncology and palliative care team members regarding their experiences.

Results: Of the patients diagnosed with stage IV cancer, 695 were deceased; of these, 347 (50\%) were referred to palliative care and $286(41 \%)$ had at least one palliative care visit. Providers made 384 unique referrals for these deceased patients, 279 (73\%) by oncologists, 41 (11\%) by primary care physicians, and 64 by other specialties. Median time between diagnosis and referral was 64 days (mean: 204, max: 1780). A quarter of patients died within 108 days, a quarter lived more than 529 days. Among the first group, 137 (85\%) survived at least 30 days; among these, $45 \%$ were referred and $45 \%$ of these referrals were within 14 days of diagnosis. Among patients in the top quartile for survival, $59 \%$ had a referral, but only $4 \%$ of those occurred within 14 days of diagnosis. Among 19 oncologists, focusing on those with $10+$ patients, referral rates ranged from $5 \%-6 \%$ ( 3 physicians) to one physician who referred $72 \%$. Interviews suggest widely varying perceptions across the oncologists. Some reported discussing palliative care at the first visit, while others described taking their cues from patients. Many physicians described patients with worsening symptoms as the best palliative care candidates but noted that, once initiated, many patients and families benefit from other services provided by the palliative care team such as goals-of-care conversations and more emotional support.

Conclusion: These data indicates wide variation in referrals to palliative care by oncologists. Interviews suggest patient preferences play a role, but the variability across oncologists presents a challenge to ensuring patients' needs and preferences are elicited and honored.
P1.01

The Acceptability, Appropriateness, Feasibility, and Effectiveness of a Cancer Survivorship Care Delivery Intervention

Sarah Birken, ${ }^{1}$ Jennifer Leeman, ${ }^{1}$ MaryBeth Grewe, ${ }^{1}$ Emily Haines, ${ }^{1}$ Darren DeWalt, ${ }^{1}$ Deborah Mayer, ${ }^{1}$ Katherine Reeder-Hayes, ${ }^{1}$ Morris Weinberger ${ }^{1}$

${ }^{I}$ The University of North Carolina at Chapel Hill, Chapel Hill, NC

Background: A large and rapidly increasing number of U.S. cancer survivors who have completed active treatment continue to seek oncology care. Low-risk survivors who continue to seek oncology care incur greater costs but experience worse care quality and outcomes than those who seek primary care. To improve the transition of low-risk survivors to primary care, we used a theorydriven, stakeholder-engaged approach to develop START (Supporting Transitions AfteR Treatment) and assess its acceptability, appropriateness, feasibility, and effectiveness in practice.

Methods: We identified desired outcomes of START based on existing frameworks for quality survivorship care and our preliminary studies. We identified facilitators and barriers to achieving outcomes through a literature review, interviews, and a focus group with stakeholders including oncology providers and administrators, primary care providers, survivors, and caregivers $(\mathrm{n}=28)$. We comprised START of strategies targeting modifiable determinants of outcomes that stakeholders deemed highly salient. Beginning in January 2019, we will collect data on START's implementation in a pilot test of its effectiveness in two large academic and one community cancer center. We will analyze data from electronic health records to assess the feasibility of identifying low-risk survivors and document changes to care processes, and we will conduct in-depth, semistructured interviews with oncology providers and staff and survivors regarding their perspectives on acceptability, appropriateness, and feasibility.

Results: START was designed to facilitate transitioning survivors through persuasive communication from clinical champions and improving information handoff from oncology providers to primary care providers and survivors. Based on our success in previous studies, we anticipate interviewing at least 5 oncology providers and staff and 2 survivors in each of the 3 pilot cancer centers $(\mathrm{n}=21)$.

Conclusion: At the conference, we will report on oncology provider and staff and survivor perceptions of START's acceptability, appropriateness, and feasibility for improving survivorship care delivery. Findings will be used to refine START and form the basis of a clinical trial to evaluate its effectiveness in improving survivorship outcomes. 
P1.02

Differential Impact of Charlson Comorbidity Index on Overall Survival in Head and Neck Cancers

\section{Steven Chang ${ }^{1}$}

\section{${ }^{1}$ Henry Ford Health System, Detroit, MI}

Background: Comorbidity burden has been associated with poorer survival in several types of cancer. We investigated the impact of the Charlson comorbidity index (CCI) on patient survival with head and neck cancer and determined a CCI cutoff based on stage and cancer site.

Methods: A retrospective cohort study was performed of all patients with any type of head and neck cancer from January 1997 to November 2016 in a single academic center in the Midwest. Data extracted included age, race, gender, primary site of malignancy, mode of treatment, clinical staging, posttreatment survival, and CCI. The value at which CCI best predicted death was identified for each site using receiver operator curves, defined as the point on the curve that maximizes sensitivity and specificity. Kaplan-Meier survival curves by CCI, stage, and site were determined.

Results: There were a total of 3720 patients. Kaplan-Meier curves demonstrated that for both early and advanced thyroid cancer, a CCI cutoff of 1 differentiated overall survival. A CCI cutoff of 3 differentiated survival in early and late laryngeal cancers, and a CCI cutoff of 1 differentiated overall survival in patients with early oral cavity cancers.

Conclusion: CCI cutoffs are clinically relevant and may inform treatment choices in thyroid, laryngeal, and oral cavity.

\section{$\mathrm{P} 1.03$}

A Centralized Mailed Program With Stepped Increases of Support and Time in Compliance With Colorectal Cancer Screening Guidelines Over 9 Years: A Randomized Trial

Beverly B. Green, ${ }^{1}$ Melissa L. Anderson, ${ }^{1}$ Andrea J. Cook, ${ }^{1}$ Jessica Chubak, ${ }^{1}$ Sharon Fuller, ${ }^{1}$ Richard T. Meenan, ${ }^{2}$ Sally W. Vernon ${ }^{3}$

${ }^{1}$ Kaiser Permanente Washington Health Research Institute, Seattle, WA; ${ }^{2}$ Kaiser Permanente Center for Health Research, Portland, OR; ${ }^{3}$ The University of Texas School of Public Health at Houston, Houston, TX

Background: Colorectal cancer (CRC) is the second-leading cause of cancer deaths in the United States. Morbidity and mortality could be reduced through higher uptake and adherence to CRC screening and follow-up. Most information on longer-term screening adherence comes from organized programs that lack a comparison group. No prior trials aimed at increasing CRC screening uptake and follow-up have evaluated outcomes beyond 5 years. Systems of Support to Increase Colorectal Cancer Screening and Follow-Up (SOS) is a randomized controlled trial comparing a centralized mailed fecal test and stepped support program to usual care. The primary outcome is time in compliance with CRC guidelines. Nine-year results will be available at the end of 2018.

Methods: SOS was implemented in an integrated health care organization in Washington State. Between 2008 and 2009, 4653 individuals 50-74 years of age were eligible and randomized to receive usual care (UC) or 1 of 3 steppedintensity interventions: mailings with a call-in number for colonoscopy and mailed fecal tests; mailings plus brief telephone assistance; or mailings and assistance plus nurse navigation. In years 3 and 5, intervention arm participants still eligible for CRC screening were randomized to stopped or continued mailings. UC participants continued to receive UC. We compared time in compliance with CRC screening over 9 years in persons assigned to any intervention vs UC. Screening tests contributed time based on national guidelines for screening intervals (fecal tests annually, sigmoidoscopy every 5 years, colonoscopy every 10 years). Active intervention-arm patients still eligible for CRC screening continued to receive stepped-intensity mailed and navigation interventions. Nineyear results will be available at the end of 2018.

Results: All participants contributed data but were censored at disenrollment, death, age 76, or CRC diagnosis. Compared with UC participants, intervention participants had 31\% more adjusted time in compliance with CRC guidelines (incidence rate ratio: 1.31 ; 95\% CI: $1.25-1.37$; coveredtime: $47.5 \%$ vs $62.1 \%$. Fecal testing accounted for almost all additional covered-time.

Conclusion: In a health care organization with clinic-based activities to increase CRC screening, a centralized program led to increased CRC screening adherence over 5 years. Longer-term data on screening adherence and its impact on CRC outcomes will be presented.

\section{P1.04 \\ Provider, Patient, and Family Caregiver Perspectives on Adopting an EHR-Integrated Web- Based Tool to Coordinate Oncology Care}

Christian Adonizio, ${ }^{1}$ Lisa Bailey-Davis, ${ }^{1}$ Chelsie Hauer, ${ }^{1}$ Amanda Milo, ${ }^{1}$ Mark Wojtowicz ${ }^{1}$

\section{${ }^{1}$ Geisinger Health, Danville, $P A$}

Background: Technological innovations allow for the integration of multisector data with the potential to relieve pain points experienced by patients and family caregivers during cancer care that often require scheduling and communication, identifying and understanding the role of health care team members, and managing medications. Engaging clinicians and a panel of patients/caregivers, we designed, developed, and deployed a web-based tool to integrate electronic health record (EHR) data with patient/ caregiver personal characteristics and events, their care partner team, and their personal calendars, with the objective 
to evaluate utility and usability of a minimally viable product. Methods: Patient/caregiver dyads were recruited to use the tool during their hematology oncology and/or radiation oncology treatment. Participants received training on the tool, and patients and caregivers were reimbursed for their time. Observations and semi-structured interviews were conducted with patients $(n=29)$, caregivers $(n=23)$, and providers $(n=8)$. Adoption factors evaluated included knowledge, persuasion, implementation, and confirmation.

Results: Patients and caregivers learned about the tool from staff, yet providers who directly endorsed the tool persuaded use. Patients/caregivers experienced challenges accessing the tool via the EHR patient portal. Personalization features enabled patients/caregivers to add photos and interests and view health care team profiles. Integration of multiple calendars worked well to coordinate oncology care and personal activities. Medication management and message features were underutilized. Providers emphasized the need to introduce the tool early in care through a synthesized HER-integrated dashboard. Providers confirmed the utility of the tool in helping to establish a holistic approach to family-centered care. Patients/ caregivers confirmed tool's utility in easing communications and recognizing health care team members.

Conclusion: The web-based tool offers the potential to address patient and family caregiver stress and burden related to a variety of tasks and engage clinicians to improve the quality of family-centered care that recognizes the important contributions of caregivers. Future research would rigorously evaluate care coordination with attention to patient, caregiver, and provider outcomes.

\section{P1.05}

\section{Reasons for Lack of Referral to Colonoscopy After an Abnormal FIT Result in a Community Health Center Network}

\author{
Ian Neff, ${ }^{1}$ Victoria Gawlik, ${ }^{2}$ Michael Kwon, ${ }^{2}$ Amanda \\ Petrik, ${ }^{1}$ Jamie Thompson, ${ }^{1}$ Matthew Slaughter, ${ }^{1}$ Gloria \\ Coronado ${ }^{1}$
}

${ }^{1}$ Kaiser Permanente Center for Health Research, Portland, OR; ${ }^{2}$ Sea Mar Community Health Centers, Seattle, WA

Background: Colorectal cancer (CRC) is the second most frequent cause of cancer-related death in the United States. Screening for CRC with fecal immunochemical tests (FIT) is an effective, noninvasive, and inexpensive way to reduce mortality. However, lack of timely colonoscopy follow-up for abnormal FIT results diminishes the benefit of using FIT as a screening modality. Screening rates, especially in underserved populations, continue to lag behind guidelines, and one reason is lack of referral to colonoscopy from primary care.

Methods: We identified 144 patients who had a positive FIT result but did not receive a referral for diagnostic colonoscopy. One author (I.N.) completed a retrospective chart review using a data collection tool developed by the research team and guided by a literature review of studies examining CRC screening in medically underserved settings. Variables collected included demographic data, comorbidities, and social determinants of health as informed by the literature. The reviewer identified any reasons for lack of referral documented in the chart by health care professionals.

Results: There are consistent emerging trends within these data. About half of patients without referral have a documented refusal or were lost to follow-up. Patients in this sample are typically uninsured or on Medicaid, speak a language other than English, and/or have documented mental illnesses or substance abuse. Some patients lack referrals but are already established with gastroenterologists or had recent colonoscopies. Finally, about one-fourth of the charts reviewed show no documentation of a discussion of abnormal FIT.

Conclusion: Results of chart review and lessons from the process have implications for future research and practice changes. This study highlights the need for greater coordination between primary care and gastrointestinal practices. The lack of interoperability between the two settings is a barrier to both research and care delivery. The frequency of patients lacking follow-up or being inappropriately screened shows a need for better coordination. There may be an opportunity to improve CRC screening for people with diagnosed mental illness as well, which needs further study. There is likely a role for patient navigation in resolving barriers associated with underinsurance or language.

\section{P1.06 \\ Breast Cancer Screening and Risk-Reducing Surgeries for Patients at High Risk for Breast and Ovarian Cancer at Kaiser Permanente Mid-Atlantic States}

Monica Ter-Minassian, ${ }^{1}$ Kala Visvanathan, ${ }^{2}$ Celeena Jefferson, ${ }^{1}$ Marcy Schaeffer, ${ }^{2}$ Pim Suwannarat ${ }^{1}$

${ }^{1}$ Kaiser Permanente Mid-Atlantic States, Rockville, MD; ${ }^{2} J o h n s$ Hopkins University, Baltimore, MD

Background: Current U.S. guidelines recommend more intensive breast cancer screening and preventive strategies for patients with a known pathogenic $B R C A 1 / 2$ mutation or a lifetime risk more than $20 \%$ based on risk prediction models accounting for personal and family history. These patients are at high risk for breast and ovarian cancer (HRBOC). Guidelines recommend that yearly mammogram alternating with magnetic resonance imaging (MRI) screening should be considered as early as 30 years old. Furthermore, BRCA1/2 mutation carriers should consider bilateral mastectomy and bilateral oophorectomy after age 35 . It is unclear what the uptake of screening and risk-reducing strategies are after recommendations by genetic services at Kaiser Permanente Mid-Atlantic States (KPMAS) for a HRBOC diagnosis.

Methods: We retrospectively studied female patients diagnosed 
as HRBOC (regardless of prior cancer) and/or tested for $B R C A$ mutations by KPMAS genetics between 2005 and 2016. We identified cancer diagnoses, mammogram and MRI screening, mastectomies, and oophorectomies through ICD diagnosis or CPT procedure codes during the study period.

Results: Our cohort included 813 women with a HRBOC diagnosis; median age at diagnosis was 51 years; racial makeup was $45 \%$ white, $38 \%$ black, and $15 \%$ other race. Since genetic services for cancer at KPMAS were established recently, 98\% of visits occurred after January 1, 2013. Most cancers occurred prior to the genetics visit 513 of 527 breast and 55 of 57 ovarian cancer diagnoses. Only 4 prophylactic mastectomies and 89 prophylactic oophorectomies were identified. For 228 patients who were 30-75 years old, breast cancer-free at time of HRBOC diagnosis, and members over 6 months, $158(69 \%)$ had at least 1 screening test (mammogram/MRI) after the genetics visit; of these, 65 had 2-3 subsequent tests and 3 had 4-6 tests. The median time to the latest screen after HRBOC diagnosis was 14.3 months, (range: 2 days to 39 months).

Conclusion: The majority of women visited genetic services after a diagnosis of breast or ovarian cancer. Patients received prophylactic oophorectomies more often than prophylactic mastectomies. Screening in cancer-free patients with a HRBOC diagnosis appears to be limited to $1-3$ screens postdiagnostic visit. Our findings suggest that earlier detection of patients at high risk for breast and ovarian cancer, as well as closer monitoring, is needed.

\section{P1.07}

Positive Predictive Value and Sensitivity of ICD-9 Codes for Childhood Leukemia in Integrated Health Plans

Sheila Weinmann, ${ }^{1,2}$ Melanie Francisco, ${ }^{1}$ Erin Bowles, ${ }^{3}$ Robert T. Greenlee, ${ }^{4}$ Alanna Rahm, ${ }^{5}$ Marilyn Kwan, ${ }^{6}$ Rebecca Smith-Bindman, ${ }^{7}$ Natasha Stout, ${ }^{8}$ Jason Pole, ${ }^{9}$ Wesley Bolch, ${ }^{10}$ James Duncan, ${ }^{11}$ Lawrence Kushi, ${ }^{6}$ Diana Miglioretti ${ }^{12}$

${ }^{1}$ Kaiser Permanente Center for Health Research, Portland, OR; 'Kaiser Permanente Center for Health Research, Honolulu, HI; ${ }^{3}$ Kaiser Permanente Washington Health Research Institute, Seattle, WA; ${ }^{4}$ Marshfield Clinic Research Foundation, Marshfield, WI; ${ }^{5}$ Research, Geisinger Health, Danville, PA; ${ }^{6}$ Division of Research, Kaiser Permanente Northern California, Oakland, CA; ${ }^{7}$ University of California, San Francisco, San Francisco, CA; ${ }^{8}$ Department of Population Medicine, Harvard Medical School and Harvard Pilgrim Health Care, Boston, MA; ${ }^{9}$ Pediatric Oncology Group of Ontario, Toronto, Canada; ${ }^{10}$ Department of Biomedical Engineering, University of Florida, Gainesville, FL; ${ }^{11}$ Division of Interventional Radiology, Washington University in St. Louis, St. Louis, MO; ${ }^{12}$ University of California Davis School of Medicine, Davis, CA
Background: Tumor registries are excellent sources for identifying leukemia cases for research, but they may be unavailable or incomplete for some health care populations. ICD-9 diagnosis codes from administrative databases may be an alternative; however, their accuracy and completeness are unknown. We calculated positive predictive value (PPV) and sensitivity of ICD-9 codes for childhood leukemia case identification.

Methods: In the Radiation-Induced Cancer study, 6 U.S. integrated health plans examined and validated ICD-9 diagnosis codes associated with leukemia for calendar years 1996-2015. Subjects were children born in the health plans and enrolled continuously for at least 120 days (unless they died) after the date of the first leukemia ICD-9 code or tumorregistry diagnosis date. We counted the number of leukemiarelated codes per subject within 120 days after the first code. We classified ICD-9 codes as specific leukemia codes, myelodysplastic syndrome/myeloproliferative disorder codes, and nonspecific codes for leukemia and related diseases. The gold standard was leukemia identified from health plan tumor registries and/or manual medical record review. We calculated PPV and sensitivity by the number of ICD-9 codes in the 120day period, overall and stratified by code categories, sites, treatment codes, and demographic factors.

Results: In a population of 857,760 subjects, 652 subjects had at least one ICD-9 code of interest and sufficient membership duration; 293 (45\%) were validated as leukemia. Among validated leukemia cases $(\mathrm{n}=295), 97 \%$ had at least 1 specific leukemia code, $2 \%$ had codes in other categories but no specific leukemia code, and $1 \%$ were in a tumor registry but had no ICD-9 codes of interest. Of those with nonspecific codes only, $2 \%$ were leukemia. Requiring 4 or more ICD9 codes gave good PPV and sensitivity. PPV for 4 or more codes was $88 \%$ for all codes combined and $97 \%$ for leukemiaspecific codes (range: $84 \%-100 \%$ by site). The sensitivity for 4 or more codes was $94 \%$ for all codes combined and $92 \%$ for leukemia-specific codes (range: $83 \%-94 \%$ by site).

Conclusion: Requiring at least 4 ICD-9 leukemia-specific codes within 120 days after the date of the first code maximizes PPV and sensitivity for identifying most cases of childhood leukemia.

\section{P1.08 \\ Budget Impact of Adopting PARP Inhibitors as Maintenance Therapy for Patients With Recurrent Ovarian Cancer}

\section{Lei $\mathrm{Wu},{ }^{1}$ Lixian Zhong ${ }^{1}$ \\ ${ }^{1}$ Texas A\&M University, College Station, TX}

Background: Ovarian cancer is one of the leading causes of death in women and accounts for an estimated 14,070 deaths in the U.S. in 2018. Recently, a new class of therapeutic agent, the poly (ADP-ribose) polymerase (PARP) inhibitor, has been approved by the Food and Drug Administration 
to be used in advanced ovarian cancer patients who have gone through platinum-based chemotherapy and relapsed. PARP inhibitors have been shown to significantly extend progression-free survivals yet are priced at over $\$ 10 \mathrm{~K}$ (WAC price) a month. This study aimed to evaluate the budget impact of adopting these therapies in a hypothetical health plan from a U.S. third-party payer perspective.

Methods: We constructed a budget impact model to assess the additional per member per month (PMPM) costs associated with the introduction of two PARP inhibitors: niraparib and olaparib. The model assessed both pharmacy costs and medical costs. Pharmacy costs included adjusted drug costs, coinsurance and dispensing fees. Medical costs included costs associated with disease monitoring and management of adverse events from the treatment. Epidemiological data from literature were used to estimate the target population size. The analysis used 1-year time frame, and patients were assumed on treatment until disease progression or death. All costs were computed in 2017 U.S. dollars. One-way sensitivity analyses were conducted to evaluate the model robustness.

Results: In a hypothetical plan of 1 million members, 206 patients were estimated to be potential candidates for niraparib or olaparib maintenance treatment after applying all epidemiological parameters. At listed WAC prices of $\$ 14,750$ for niraparib and $\$ 13,482$ for olaparib, budget impacts of these two drugs were \$0.169 PMPM and \$0.156 PMPM, respectively, most of which were contributed by pharmacy costs. Sensitivity analyses suggested that assumptions around market share, platinum-sensitive rate after the first treatment, and WAC prices affected results most.

Conclusion: The new PARP inhibitors niraparib and olaparib are associated with high costs for patients on treatment. However, relatively few patients are eligible for the treatment due to the relatively low prevalence of the disease state and low initial market penetration. From a U.S. payer's perspective, the overall budget impact of these two new treatment options is moderate in the first 12-month period.

\section{CHILD AND ADOLESCENT HEALTH}

\section{P4.01 \\ Validation of the Pediatric Appendicitis Risk Calculator in the Community Emergency Department Setting}

Dustin W. Ballard, ${ }^{1}$ Laura E. Simon, ${ }^{1}$ Adina S. Rauchwerger, ${ }^{1}$ David R. Vinson, ${ }^{1}$ Gabriela VazquezBenitez, ${ }^{2}$ E. Margaret Warton, ${ }^{1}$ Dale M. Cotton, ${ }^{1}$ Elyse O. Kharbanda, ${ }^{2}$ Anupam B. Kharbanda ${ }^{3}$

${ }^{1}$ Kaiser Permanente, Oakland, CA; ${ }^{2}$ HealthPartners, Minneapolis, MN; ${ }^{3}$ Children's Hospitals of Minnesota, Minneapolis, $M N$
Background: Pediatric appendicitis is a challenging diagnosis in the emergency department (ED). Established prediction rules, such as the pediatric appendicitis score (PAS), may encourage overutilization of diagnostic imaging. The pediatric appendicitis risk calculator (pARC) was recently derived and validated in a retrospective cohort of children's hospital patients. We prospectively evaluated the performance of pARC in a community setting.

Methods: As part of a larger electronic clinical decision support trial for pediatric patients with abdominal pain, we conducted a multicenter, prospective validation study from October 1, 2016, to April 30, 2018, in 11 community EDs within an integrated delivery system. To collect clinical variables, treating ED physicians enrolled patients 5-20.9 years of age with a chief complaint of abdominal pain and $<5$ days of right-sided or diffuse abdominal pain via a web-based application embedded in the electronic health record (EHR). Patients with abdominal trauma, pregnancy, and known history of appendectomy were excluded. Outcomes (appendicitis, perforation, negative appendectomy rate, missed appendicitis) were identified from automated EHR data and confirmed by manual chart review. Descriptive results are reported by pARC risk category, and validation analyses included discrimination (area under the curve [AUC] of receiver operating characteristic curve) and calibration (goodness of fit) of the PAS and pARC.

Results: We enrolled 2089 eligible patients (46.1\% male) with a median age of 12.4 years (interquartile range: 9-16). Appendicitis was confirmed in 353 (16.9\%) patients; 52 (14.7\%) had perforation, negative appendectomy rate was $6.1 \%$, and missed appendicitis occurred in $10(0.5 \%)$ patients. The distribution of pARC was: $53.8 \%$ at low risk $(<15 \%) ; 38.9 \%$ at intermediate risk $(15 \%-74 \%)$; and $7.4 \%$ at high risk $(>74 \%)$. Appendicitis rates ranged from $2.0 \%$ in the low-risk group to $75.3 \%$ in the high-risk group. The pARC had an AUC of 0.89 (95\% CI: $0.87-$ $0.91)$ vs 0.80 (95\% CI: $0.77-0.82)$ for PAS. Calibration testing resulted in a Hosmer-Lemeshow goodness of fit of $\mathrm{P}=0.16$.

Conclusion: The pARC accurately quantified appendicitis risk in children presenting to community EDs with abdominal pain and outperformed the PAS. Over half of the patients in this community validation sample could be accurately classified as low or high risk for appendicitis. Incorporation of the $\mathrm{pARC}$ into clinical practice may help guide imaging and treatment decisions. 


\section{GENETICS, GENOMICS, AND PRECISION MEDICINE}

P7.01

The New CESR Virtual Data Warehouse Molecular
Marker Result Database (Genetic Tests and Tissue
Markers) - Scheduled to Launch by the End of
2019

Don Bachman, ${ }^{1}$ Andrea Burnett-Hartman, ${ }^{2}$ Susan Gouchoe, ${ }^{3}$ Lawrence Kushi, ${ }^{4}$

${ }^{I}$ Kaiser Permanente Center for Health Research, Portland, OR; 'Institute for Health Research, Kaiser Permanente Colorado, Denver, CO; ${ }^{3}$ Genomic Strategy \& Implementation, National Health Plan and Hospital Quality, Kaiser Permanente, Oakland, CA; ${ }^{4}$ Division of Research, Kaiser Permanente Northern California, Oakland, CA

Background: The Kaiser Permanente (KP) Center for Effectiveness and Safety Research (CESR) is a national research collaborative comprising the research centers from the $8 \mathrm{KP}$ regions. Most KP member molecular marker (genetic and tissue marker) test results are evaluated by 15 outside vendors and by internal staff within $3 \mathrm{KP}$ regions. While these results are viewable in the electronic medical record, the vast majority are not currently stored in discrete data fields for population analysis. So, the CESR Data Coordinating Center (DCC) convened a workgroup consisting of investigators, genetic counselors, research network consultants, programmers, and the KP genomic strategy \& implementation department to create a virtual data warehouse (VDW) molecular marker result database at each KP region.

Methods: The molecular marker workgroup designed the CESR VDW molecular marker results data dictionary, consistent with the 2017 Health Level Seven International specifications whenever possible. The tables include positive, negative, and inconclusive results. The genomic strategy \& implementation department programmers link the test result records to appropriate KP member and region identifiers. The KP DCC then codes an ETL (extract, transform, load) program that transforms each data source to the CESR VDW molecular marker specifications. The research data warehouse staff from each region will execute this ETL program to pull their own site's data. The VDW molecular marker database consists of 3 tables within each region's VDW: an order-level table; a marker-level table; and a variant-level table.

Results: While the key variables are being populated by the vendors, not all requested fields are available from each one. At the time of the abstract, we have collected at least some data from 5 of the 15 main vendors.

Conclusion: This CESR VDW molecular marker database is designed to facilitate single- and multiregion genetic research. It contains results and interpretations at the order, marker, and variant levels; includes positive, negative, and inconclusive results; and is scheduled to be ready for projects by the end of 2019 .

P7.02

Predictors of Current Posttraumatic Stress Disorder Among Deployed Veterans: Significance of Predisposition, Stress Exposures, and Genetic Factors

Yirui $\mathrm{Hu},{ }^{1}$ Xin Chu, ${ }^{1}$ Thomas Urosevich, ${ }^{1}$ Stuart Hoffman, ${ }^{1} \mathrm{H}$. Lester Kirchner, ${ }^{1}$ Richard Adams, ${ }^{2}$ Ryan Dugan, ${ }^{1}$ Joseph J. Boscarino, ${ }^{3}$ Carrie Withey, ${ }^{1}$ Charles Figley, ${ }^{4}$ Joseph A. Boscarino ${ }^{1}$

${ }^{1}$ Geisinger Health, Danville, PA; ${ }^{2}$ Kent State University, Kent, OH; ${ }^{3}$ William James College, Newton, MA; ${ }^{4}$ Tulane University, New Orleans, LA

Background: Previously (2012) we reported that a genetic risk model improved posttraumatic stress disorder (PTSD) predictions among a trauma-exposed civilian population (69\% female; mean age of 55.4 years [standard deviation (SD): 13.4]). We sought to assess this prediction model among a trauma-exposed U.S. military population.

Methods: We examined postdeployment PTSD status among a cross-sectional sample of 1074 community-based veterans of Vietnam, Persian Gulf, Iraq/Afghanistan, and other recent conflicts, who were receiving care in a large nonVA multihospital system in Pennsylvania. Approximately $95 \%$ of these veterans were male (mean population age: 61.4 years [SD: 12.1]). To avoid confounding due to genetic admixture, non-Caucasian veterans $(\mathrm{n}=40)$ were excluded.

Results: $7.1 \%$ of veterans (95\% CI: 5.6-8.8) met DSM-5 criteria for current PTSD. The mean risk-allele count was significantly higher among PTSD cases vs non-cases (3.9 vs $3.5, \mathrm{P}=0.027$ ). The PTSD risk variants studied were within genetic loci previously associated with PTSD, including CRHR1, CHRNA5, RORA, and FKBP5 genetic variants, which were used to calculate a total PTSD risk score (range: 0-8, mean: 3.6, SD: 1.4). The final logistic regression prediction model included the genetic risk score, plus demographic factors (age, sex), trauma exposures (combat, lifetime trauma exposures), concussion history, current traumatic brain injury symptoms, current life stressors, and history of attention deficit disorders. Significant variables in the final PTSD model included current traumatic brain injury symptoms (odds ratio [OR]: 7.5, $\mathrm{P}<0.001$ ), history of attention deficit disorders (OR: 3.0, $\mathrm{P}<0.001$ ), current life stressors (OR: 3.0, $\mathrm{P}<0.001$ ), lifetime trauma exposure (OR: 2.0, $\mathrm{P}=0.021$ ), concussion history (OR: $2.1, \mathrm{P}=0.021$ ), and PTSD risk allele count (OR: 1.3, $\mathrm{P}=0.021)$. Inconsistent with previous research with nonveterans, no interaction effects were detected for trauma exposures by genetic risk score.

Conclusion: Our study partially replicated previous results among trauma-exposed civilians. After deployment, both 
warzone and non-warzone factors predicted current PTSD among U.S. veterans seen in non-VA facilities, including a genetic risk score for PTSD. However, no interaction effects were detected for trauma exposures by genetic risk score. Further research is planned.

\section{MATERNAL AND PERINATAL CARE}

\section{P11.01 \\ Bias in Self-Reported Prepregnancy Weight Across Maternal and Clinical Characteristics}

Joanna Bulkley, ${ }^{1}$ Kimberly Vesco, ${ }^{1}$ William Callaghan, ${ }^{2}$ Padmavati Dandamudi, ${ }^{1}$ Ashley Stoneburner, ${ }^{3}$ Michael Leo, ${ }^{1}$ Andrea Sharma ${ }^{2}$

${ }^{1}$ Kaiser Permanente Center for Health Research, Portland, OR; ${ }^{2}$ Centers for Disease Control and Prevention, Atlanta, GA; ${ }^{3}$ Nurse-Family Partnership, Denver, CO

Background: Prepregnancy body mass index (BMI) and gestational weight gain (GWG) are known determinants of maternal and child health; both require an accurate measure of prepregnancy weight. Prepregnancy weight is typically self-reported. We used electronic health record (EHR) data to compare self-reported prepregnancy weight (SR-weight) to measured clinic weights to assess reporting bias by maternal and clinical characteristics.

Methods: Pregnant women $\geq 18$ years old who began pregnancy and started prenatal care from January 2000 to December 2010 at Kaiser Permanente Northwest were screened for this retrospective cohort study and included in the analysis if their EHR contained 1) SR-weight (obstetric questionnaire), 2) at least 2 measured weights between $\leq 365$ days prior to and $\leq 42$ days after conception, and 3 ) measured height. Covariates were extracted from the EHR and birth certificates. The measured weight closest to date of conception between 6 months prior to ( $\leq 182$ days) and $\leq 42$ days after conception was considered the "gold standard" prepregnancy weight. Generalized-estimating equations were used to assess predictors of misreport, controlling for covariates.

Results: Of 56,122 pregnancies screened, 16,227 (29\%) had the required data. Close agreement $( \pm 1 \mathrm{~kg}$ or within $2 \%$ of measured weight) between SR-weight and measured weight was $44 \%$ and $59 \%$, respectively $(\mathrm{rc}=0.99)$. Overall, SR-weight averaged $1.3 \mathrm{~kg}$ (standard deviation: 3.8) less than measured weight. The degree of underreporting was higher among women with elevated BMI, late entry into prenatal care, and a pregnancy outcome other than live birth or stillbirth $(\mathrm{P}<0.05)$. Misreport did not differ by age, race/ethnicity, marital status, education, smoking, parity, or Medicaid enrollment. Using SR-weight, BMI was correctly classified for $91 \%$ of pregnancies (range: $70 \%-98 \%$, depending on BMI category).
Conclusion: An accurate measure of prepregnancy weight is essential for clinical guidance during pregnancy and for surveillance efforts that monitor maternal health and evaluate progress of public health programs. SR-weight performs relatively well when it is used to categorize BMI, but the misreport observed may result in misclassification of GWG. Our analysis identified characteristics associated with misreport of SR-weight, which can inform understanding of bias when assessing the influence of prepregnancy BMI or GWG misclassification on associations with maternal and child health outcomes.

\section{P11.02}

Hospital Emergency Department Visits for Women Prior to Birth and After Pregnancy, 2014

\section{Geoffrey Jackson, ${ }^{1}$ Sonja Williams, ${ }^{1}$ Karishma Chari ${ }^{1}$ \\ ${ }^{1}$ National Center for Health Statistics, Hyattsville, MD}

Background: Hospital emergency departments (EDs) are commonly used by pregnant women for nonurgent care and for urgent routine care. In 2015, there were an estimated 2.3 million visits to hospital EDs by women between the ages of 15 and 64 years for complications of pregnancy, childbirth, and the puerperium (National Hospital Ambulatory Medicare Survey: 2015 Emergency Department Summary Tables). Describing maternal care in the ED setting provides insight on the care and creates new opportunities for improvement in care.

Methods: This study utilizes 2014 data from the National Hospital Care Survey (NHCS), which collects information on patients, conditions, and care received during visits to hospital inpatient and emergency departments, from a sample of hospitals across the nation. The survey collects demographic information and diagnoses and procedures related to the visit. NHCS is unique in that patient identifiers are collected, allowing patients to be tracked across hospital settings and over time. The 2014 NHCS collected hospital claims data on 1.6 million inpatient and 4.5 million ED visits from 95 hospitals throughout the United States. NHCS data are not nationally representative yet but can be used for exploratory analysis on hospital care. This study provides information on women who delivered a baby in the inpatient setting, whether they visited the sampled hospital's ED prior to or after the birth of their children, the reason for the visit, and if there were any complications related to pregnancy.

Results: The 2014 NHCS dataset contains information on women who delivered a baby in a sampled hospital's inpatient department $(\mathrm{n}=177,000)$. Of these women, approximately $15 \%$ sought care in a sampled NHCS hospital's ED prior to the birth and 9\% visited a sampled hospital's ED postdelivery. This study examines women who visited an ED for pregnancy complications by age category before or after giving birth (eg, early labor, hemorrhaging, excessive vomiting, symptoms involving the abdomen and 
pelvis, and disorders of urethra). This study also analyzes the relationship of pre- and postbirth health issues with the presence of complications during birth.

Conclusion: This complete picture of pregnancy-related hospital care can provide greater insight into pre- and postnatal care.

\section{METHODS, DESIGNS, AND ANALYTICS TOOLS}

\section{P13.01 \\ Prognosticating Outcomes and Nudging Decisions With Electronic Records in the Intensive Care Unit (PONDER-ICU) Trial: Implementation in a Learning Health System}

Katherine Courtright, ${ }^{1}$ Erich Dress, ${ }^{1}$ Jaspal Singh, ${ }^{2}$ Brian Bayes, ${ }^{1}$ Steven Brooks, ${ }^{1}$ Elizabeth Cooney, ${ }^{1}$ Vanessa Madden, ${ }^{1}$ Scott Halpern ${ }^{1}$

${ }^{1}$ University of Pennsylvania, Philadelphia, PA; ${ }^{2}$ Atrium Health System, Charlotte, NC

Background: Almost all critically ill patients wish to receive therapies that promote both the duration and quality of their lives. Yet, too often such patients' care focuses exclusively on one of these goals. The mismatch between the care that patients want and the care they receive stems, in part, from the failure of clinicians to discuss patient goals and preferences in a timely manner. The PONDER-ICU trial will test the effectiveness of two electronic health record (EHR) interventions designed to increase intensive care unit (ICU) clinicians' engagement of critically ill patients and caregivers in discussions about alternative treatment options, including care focused on comfort.

Methods: This pragmatic, stepped-wedge cluster randomized controlled trial is being conducted in 19 ICUs of 10 hospitals within the Atrium Health System. Eligible patients are identified in real time using an EHR-based algorithm with no research personnel onsite. The EHR will then automatically trigger the clinician-facing interventions. ICU clinicians will be required to 1) document a prognostic estimate of their patients' functional outcomes at 6 months, and 2) provide a justification if they choose not to offer patients the option of comfort-oriented care. We hypothesize that these simple, scalable interventions will prompt physicians to reflect more deeply on the most appropriate care for their patients. The primary outcome is hospital length of stay, with death ranked as the longest length of stay. Secondary outcomes include clinical and palliative care process-related measures

Results: The trial launched in February 2018 and began collecting control phase data at all 10 hospital sites. To date, 4 hospitals have begun the intervention phase of the trial.
This work-in-progress presentation will include preliminary data including rates of clinician adherence, responses to the interventions, and challenges faced with implementing a purely EHR-based trial.

Conclusion: The results of this trial hold great potential to affect clinical practice, as hospitals and payers are highly motivated to identify best practices that can be broadly implemented to improve quality while containing costs. In addition, the simple EHR-based interventions being tested are highly scalable - thereby facilitating adoption by hospitals and ICUs across the United States if shown to be effective.

\section{$\mathrm{P} 13.02$ \\ Development of Algorithms to Identify Pregnancy Outcomes From the Electronic Health Record}

Kajal Angras, ${ }^{1}$ Shantel Quinn, ${ }^{1}$ Dustin Hartzel, ${ }^{1}$ Grant Walter, ${ }^{1}$ A. Dhanya Mackeen ${ }^{1}$

${ }^{1}$ Geisinger Health, Danville, PA

Background: Pregnant women are counseled regarding the risk of adverse outcomes based on prepregnancy body mass index (BMI). Obesity, defined as BMI greater than or equal to $30 \mathrm{~kg} / \mathrm{m}^{2}$, places women at risk for several complications such as fetal anomalies, hypertensive disorders of pregnancy, gestational diabetes, and cesarean delivery. This study's aim was to develop algorithms, in addition to the diagnostic codes, using data from electronic health records (EHR) for identification of maternal and neonatal adverse risks outlined by specific prepregnancy BMI classes for a large retrospective cohort study.

Methods: We first developed a pregnancy algorithm using the prenatal event from the EHR. Using a combination of information from ultrasound, smart forms, and flow sheets, the dataset was then completed for any missing data. After identifying the total number of pregnant patients, exclusion criteria were applied using International Classification of Diseases (ICD) codes. We then extracted data variables that were readily available such as Apgar scores, birth weight, and gender of neonate. Prepregnancy BMI was calculated from heights and weights chosen from the value closest to the start of the pregnancy date. Outcome variables were identified by ICD codes and algorithms. Manual chart review was then conducted to validate the dataset. Adjustments were made to the algorithms to better capture the outcomes. Re-review of the charts validated the final algorithms.

Results: We showed that use of rigorous research methodology for data extraction through the development of algorithms significantly improved the detection of outcomes. Relying solely on ICD codes may compromise the accuracy of the dataset.

Conclusion: Development of algorithms, in addition to the use of ICD codes, improved the quality of the data for our large retrospective cohort study. 


\section{P13.04}

\section{Perspectives From a Coordinating Center: Lessons Learned From the Biologics and Biosimilars Collective Intelligence Consortium}

\author{
Sarah Malek, ${ }^{1}$ Katelyn King, ${ }^{1}$ Cate Lockhart, ${ }^{2}$ Aaron \\ Mendelsohn, ${ }^{1,3}$ Jeffrey Brown ${ }^{1,3}$
}

${ }^{1}$ Harvard Pilgrim Health Care Institute, Boston, MA; ${ }^{2} A M C P$ Biologics and Biosimilars Collective Intelligence Consortium, Alexandria, VA; ${ }^{3}$ Harvard Medical School, Boston, MA

Background: The Biologics and Biosimilars Collective Intelligence Consortium (BBCIC) was established in 2015 to analyze real-world evidence on safety and effectiveness of biological drugs, including both originator biologics and biosimilars. Harvard Pilgrim Health Care Institute, a Health Care Systems Research Network member, has served as the BBCIC coordinating center since BBCIC inception.

Methods: BBCIC is a multiuse, multisite distributed research network. It is a nonprofit subsidiary of the Academy of Managed Care Pharmacy (AMCP), overseen by a separate board of directors. BBCIC is funded by pharmaceutical manufacturers and in-kind health plan contributions. Research plans are developed by a science committee and approved by a planning board. BBCIC activities are contracted independently; to date, 8 workgroups have been formed. All stakeholders can contribute to research through workgroup participation. BBCIC leverages infrastructure from the Food and Drug Administration's Sentinel Initiative, specifically claims data formatted in the Sentinel Common Data Model and publicly available analytic tools.

Results: Administratively establishing the network and individual workgroups required development of statements of work (SOWs), budgets, contract execution, and institutional review board submissions for participating research data partners and additional investigators. Sufficient time is needed for start-up activities to ensure clarity and avoid later delays. SOW terminology should be consistent and deliverables defined. Expectations for dissemination, including authorship, should be predefined, and a dissemination process should be established. Infrastructure leveraging the Sentinel capabilities enabled the BBCIC to initiate research quickly. However, planning for and having adequate resources to educate and train stakeholders who varied in their knowledge of the capabilities of the analytic tools and data were identified as critical elements to ensure appropriate and efficient implementation of consortium research. Workgroups operating multiple concurrent workgroups required coordination and prioritization. Large workgroups require detailed workflows to define process and responsibilities. Smaller workgroups mitigate delays but must be balanced with the value of incorporating diverse stakeholder expertise. A central repository to share documentation and facilitate communication is crucial.

Conclusion: Managing a multiuse, multisite distributed research network is complex. Complexity is heightened with an intricate, yet transparent, governance structure that includes multiple stakeholders. Responsiveness to a fluid environment, effective communication, and clear expectations are essential.

\section{P13.05}

\section{Evidence for Nonpharmacologic Treatment for Chronic Pain: Results and Research Gaps}

Andrea Skelly, ${ }^{1}$ Roger Chou, ${ }^{2}$ Joseph Dettori, ${ }^{3}$ Judith Turner, ${ }^{4}$ Janna Friedly, ${ }^{4}$ Sean Rundell, ${ }^{4}$ Rochelle Fu, ${ }^{2}$ Erika Brodt, ${ }^{1}$ Ngoc Wasson, ${ }^{2}$ Cassandra Winter, ${ }^{1}$ Aaron Ferguson ${ }^{1}$

${ }^{1}$ Aggregate Analytics, Inc., Fircrest, WA; ${ }^{2}$ Oregon Health \& Science University, Portland, OR; ${ }^{3}$ Spectrum Research, Inc., Tacoma, WA, ${ }^{4}$ University of Washington, Seattle, WA

Background: Opioids are commonly utilized for chronic pain management. In 2016, more than 2 million Americans reported struggling with opioid use disorder and an estimated 17,087 overdose deaths involving prescription opioids occurred. Consideration of nonopioid management alternatives, including nonpharmacologic options, and assessment of the evidence for them is vital, as is the need to address evidence gaps.

Methods: Randomized controlled trials of noninvasive, nonpharmacological treatments for 5 chronic pain conditions (low back pain, neck pain, fibromyalgia, osteoarthritis, and tension headache) that reported results for at least 1 month postintervention were included. Results were summarized for function and pain and focused on persistence of effects.

Results: A total of 202 trials were included. Interventions associated with slight to moderate improvement of function and/or pain for at least 1 month postintervention were 1) for chronic low back pain: exercise, psychological therapies (cognitive behavioral therapy), spinal manipulation, low-level laser therapy, massage, mindfulness-based stress reduction, yoga, acupuncture, multidisciplinary rehabilitation; 2) for chronic neck pain: exercise, lowlevel laser, Alexander Technique, acupuncture; 3) for knee osteoarthritis: exercise, ultrasound; for hip osteoarthritis: exercise, manual therapies; 4) for fibromyalgia: exercise, cognitive behavioral therapy, myofascial release, tai chi, qigong, acupuncture, multidisciplinary rehabilitation; and 5) for chronic tension headache: spinal manipulation. The level of supporting evidence varied from condition to condition. There tended to be more evidence for the effects of interventions on pain than for function; effects on function were generally smaller. No evidence suggesting serious harms was identified. Studies comparing interventions with pharmacological or active interventions were few. Longterm evidence and data on harms were sparse.

Conclusion: Our findings provide some support for strategies and policies that focus on nonpharmacological 
therapies for specific chronic pain conditions, but evidence gaps are many. Studies with long-term follow-up that focus on effect sustainability; use methods to enhance recruitment, retention, and adherence; employ standardize protocols; and evaluate comparisons with active interventions are needed. This project was funded under Contract No. HHSA290201500009I from the Agency for Healthcare Research and Quality (AHRQ), U.S. Department of Health and Human Services (HHS). The authors of this abstract are responsible for its content. Statements in the abstract do not necessarily represent the official views of or imply endorsement by AHRQ or HHS.

\section{P13.06}

Demonstrating Impact of Design and Evaluation Choices on Estimation of Effectiveness of Transition-of-Care Program

\author{
Shruti Vaidya, ${ }^{1}$ Sylvia Sudat, ${ }^{1}$ Alice Pressman ${ }^{1}$ \\ ${ }^{1}$ Sutter Health, Walnut Creek, CA
}

Background: Health care interventions are frequently implemented without evaluation in mind. When the time comes to measure effectiveness, this creates analytical challenges, and the choices made during the evaluation can introduce limitations on the interpretation of final results. We describe the challenges faced during an evaluation of a hospital-based transition-of-care (TOC) program using electronic health records (EHR) and show the impact of these challenges on our ability to effectively evaluate the program.

Methods: The TOC program was implemented at Sutter Health, a large health care system in northern California. We developed EHR-based inclusion/exclusion criteria to mimic the care team's process of identifying patient hospitalizations for intervention. To understand the extent of disagreement, we compared patient hospitalizations that actually received an intervention (TOC intervention cohort) with hospitalizations identified using EHR-based criteria. We then used a matching algorithm to create controls for 3 treatment cohorts: a cohort identified using only EHR-based criteria $(\mathrm{C}-1, \mathrm{n}=1,558)$; a subset of the TOC intervention cohort that overlaps with EHR-based criteria $(C-2, n=375)$; and the actual TOC intervention cohort $(C-3, n=565)$.

Results: The cohort identified using EHR-based criteria captured only $66 \%$ of the actual interventions. This introduced challenges for defining controls post hoc for valid comparison. Although we could identify matched controls for $92 \%$ of treatment cohort C-1, only $24 \%$ of actual interventions qualified on EHR-based criteria. For C-2, the matching algorithm identified controls for $89 \%$ of the treatment cohort, but this left out $34 \%$ of actual interventions. Finally, when we considered cohort C-3, the differences between EHR-based criteria and the actual intervention cohort became evident, as the matching algorithm found controls for only $71 \%$ of those treated.

Conclusion: In our study, a lack of agreement between the EHR-based cohort and actual intervention criteria made a confident evaluation nearly impossible. Although these TOC interventions were beneficial to individual patients, we were unable to demonstrate measurable outcomes due to study design limitations. We believe that the methods to illustrate and showcase patient stories and individual patient outcomes are warranted in situations for which delivering the best clinical care does not lend itself to rigorous evaluation.

\section{P13.07 \\ Identification of Persons With Disorders of Sex Development in Electronic Medical Records: Methodology and Results}

Michael Goodman, ${ }^{1}$ Rebecca Nash, ${ }^{1}$ Andrew Bradlyn, ${ }^{2}$ Lee Cromwell, ${ }^{2}$ Melissa Gardner, ${ }^{3}$ Darios Getahun, ${ }^{4}$ Trenton Hoffman, ${ }^{1}$ Theresa Im, ${ }^{4}$ Timothy Lash, ${ }^{1}$ Peter Lee, ${ }^{5}$ Brandi Robinson, ${ }^{2}$ Douglas Roblin, ${ }^{6}$ Christine Truong, ${ }^{6}$ Suma Vupputuri, ${ }^{6}$ Rami Yacoub, ${ }^{1}$ David Sandberg ${ }^{3}$

${ }^{1}$ Emory University, Atlanta, GA; ${ }^{2}$ Kaiser Permanente Georgia, Atlanta, GA; ${ }^{3}$ University of Michigan, Ann Arbor, MI; ${ }^{4}$ Kaiser Permanente Southern California, Pasadena, CA; ${ }^{5}$ Pennsylvania State University, University Park, PA; ${ }^{6}$ Kaiser Permanente Mid-Atlantic States, Rockville, MD

Background: The terms "intersex" and "disorders of sex development" (DSD) refer to a broad and heterogeneous group of conditions in which development of chromosomal, gonadal, or anatomic sex is atypical. What constitutes optimal DSD care is a matter of ongoing debate within and across various constituencies representing health care providers, advocacy groups and DSD patients. A direct assessment of how patients are evaluated, the types of treatments they receive, and the outcomes of these treatments requires large longitudinal studies representing the full range of DSD diagnoses.

Methods: A computer program scanned the electronic medical record (EMR) from Kaiser Permanente (KP) Georgia, KP Southern California, and KP Mid-Atlantic States from January 2006 through December 2017 for 131 potentially relevant diagnostic codes. As some patients with DSD may not have specific diagnoses documented in the EMR, we also searched free-text clinical notes for the presence of 143 keywords that may be indicative of the conditions of interest. Code and keyword lists were developed based on consultations with health care providers and researchers with specific and relevant expertise in this area; the lists were designed to be broad and included keywords not necessarily specific to DSD. This was done to minimize the probability of missing an eligible participant, but with an understanding that most of the candidates will turn out to be noneligible on a more detailed record review. 
Results: Preliminary results from KP Georgia identified a DSD cohort of 7605 members with at least one diagnostic code or keyword of interest - 6357 of those had keywords only, and 1248 had both diagnostic codes and keywords. In contrast, 1313 candidates had diagnoses or keywords consistent with true DSD after record review. Keywords alone could contribute $30 \%$ of total cohort but consist mostly of persons with distal hypospadias and bicornuate uterus (non-DSD). Very few true DSD patients were identified without diagnostic codes, and none were recently diagnosed. Additional results of the full cohort will be presented.

Conclusion: The methodology used in this study is relatively low-cost, rapid, and effective. It will allow the examination of specific, and sometimes rare, DSD, which have been difficult to study in the past.

\section{POPULATION, COMMUNITY, AND WORKSITE HEALTH}

\section{P15.01 \\ Comparison of Flu Immunization Prevalence Based on Electronic Health Records vs Survey Data}

\section{Nancy P. Gordon, ${ }^{1}$ Ousseny Zerbo ${ }^{1}$ \\ ${ }^{1}$ Division of Research, Kaiser Permanente Northern California, Oakland, CA}

Background: Quality reporting for adult flu immunization may shift from survey-based to electronic health record (EHR)-based data. We wanted to learn how flu immunization prevalence (PFI) estimated from a member survey (S-PFI) compared to PFI based on EHR data (E-PHI) and the extent to which E-PHI might underestimate actual flu immunization coverage due to nonreported out-of-plan immunizations.

Methods: We estimated PFI and percentages of immunized adults, age 20-89 years, who were immunized outside Kaiser Permanente Northern California (KPNC) using weighted data from the 2014/2015 KPNC Member Health Survey $(n=15,678)$. E-PFI for the 2015/2016 flu season was produced for a cohort of KPNC adults, age 20-89, who were members from October 2015 to December $2016(n=2,370,277)$. S-PFI and E-PHI were compared for all adults, those 20-64 years old, 4 age strata $(20-34,35-49,50-64,65-89)$, and those at "high" (diabetes, prediabetes, heart disease, chronic obstructive pulmonary disease, asthma, or body mass index of $\geq 40$ ) vs "average" risk (none of these conditions). We also compared E-PFI by race/ethnicity and sex within age strata. Results: S-PFI was higher than E-PFI for age groups 20-89 (62.8\% vs $42.4 \%), 20-64$ (57.6\% vs $33.6 \%)$, all age strata (49.7\% vs $22.7 \%, 57.6 \%$ vs $32.9 \%, 65.1 \%$ vs $42.8 \%, 84.2 \%$ vs $72.0 \%$, respectively), and for high (65.6\% vs $55.7 \%)$ and average risk (44.1\% vs 30.0\%) adults less than 65 years of age. Blacks and Latinos had lower overall E-PFI than whites and Asians. Age-sex stratification narrowed Latino-white (but not black-white) differences in E-PFI. Based on selfreported survey data, we estimated that of those who were immunized, approximately $20 \%$ of adults $20-34$ years old, $15 \%$ of those $35-49$ years old, $12 \%$ of those $50-64$ years old, and $4 \%$ of those $65-89$ years old were immunized outside KPNC and would not have EHR documentation.

Conclusion: EHR-based flu immunization prevalence was significantly lower than survey-based prevalence. Survey data suggest that without outreach to encourage health plan members to report out-of-plan flu immunization, EHR documentation will likely underestimate flu immunization coverage, especially among adults younger than age 65 .

P15.02

The Health and Well-Being of HealthPartners' English-, Hmong-, and Spanish-Speaking Members

Thomas Kottke, ${ }^{1}$ Jason Gallagher, ${ }^{1}$ Marcia Lowry, ${ }^{1}$ Sachin Rauri, ${ }^{1}$ Juliana Tillema, ${ }^{1}$ Jeanette Y. Ziegenfuss, ${ }^{1}$ Nico Pronk, ${ }^{1}$ Susan Knudson ${ }^{1}$

\section{${ }^{1}$ HealthPartners, Minneapolis, MN}

Background: HealthPartners has developed Summary Measures of Health and Well-Being that comprise current health, future health, and well-being. Current health is assessed from claims; the other two components are assessed by survey. Future health comprises 7 items (tobacco use, fruits and vegetables, physical activity, alcohol, sleep, healthy thinking, and preventive services). Our measure of well-being is response to the question, "How satisfied are you with your life?" To better meet the health and well-being needs of its culturally diverse membership, HealthPartners has surveyed a sample of its adult English, Hmong, and Spanish speakers and analyzed their claims data to identify similarities and differences. These observations will be used to strategically inform program development.

Methods: We analyzed claims and surveys for the following dates: July 1, 2016, to June 30, 2017, for English and Spanish speakers; and January 1, 2016, to December 31, 2016, for Hmong speakers. Survey respondents and response rates were 7699 (46\%) English speakers, 341 (40\%) Hmong speakers, and 871 (49\%) Spanish speakers.

Results: The current health score (calculated as 1 - mean disability-adjusted life years * 100) was 69 for English, 72 for Hmong, and 68 for Spanish speakers. The most burdensome conditions were musculoskeletal, psychosocial, and neurologic for all 3 groups. We found that Hmong members suffer an unusually high burden from stroke. In all 3 groups better nutrition offered the greatest opportunity for improvement in the future health score, followed by healthy thinking. While $42 \%, 41 \%$, and $60 \%$ of English-, Hmongand Spanish-speaking respondents reported high levels of life satisfaction, far more Hmong (26\%) reported very low levels relative to English (13\%) and Spanish (9\%) speakers. Conclusion: While there are important differences in 
current and future health among the 3 groups and the life satisfaction data must be interpreted cautiously because of cultural differences among the respondents, the threats to current health, the threats to future health, and levels of wellbeing are more similar than different. This finding suggests that health promoting programs can address the same issues in all 3 communities. However, interventions must be tailored to each to be culturally meaningful.

\section{P15.05}

\section{Epidemiological Burden of Systemic Sclerosis - A Systematic Review}

\author{
Lixian Zhong, ${ }^{1}$ Melinda Pope, ${ }^{1}$ Ye Shen, ${ }^{2}$ Jose \\ Hernandez, ${ }^{1}$ Lin $\mathrm{Wu}^{3}$ \\ ${ }^{1}$ Texas A\&M University, College Station, $T X ;{ }^{2}$ University of \\ Georgia, Athens, GA; ${ }^{3}$ The University of Tennessee Health \\ Science Center, Memphis, TN
}

Background: Systemic sclerosis is a debilitating autoimmune disease of the connective tissue currently with no cure. Systemic sclerosis is rare but present throughout the world. Due to improved clinical awareness and diagnosis, the prevalence and incidence of systemic sclerosis increased significantly in the 20th century during the 50s and 90 s. Estimates of prevalence and incidence are challenging due to the rarity of the disease and the wide spectrum of clinical manifestations. They are further complicated by different diagnosis criteria, clinical practice, study design, timing, etc. This study aimed to assess the recent prevalence and incidence of systematic sclerosis across the world via a systematic literature review.
Methods: Using a systematic search strategy, PubMed/ MEDLINE and Embase were searched to identify relevant studies published from January 2006 to June 2016. Two independent reviewers evaluated studies for inclusion based on inclusion/exclusion criteria and performed data extraction. The review was conducted and reported according to Preferred Reporting Items for Systematic Review and Meta-Analysis (PRISMA) statement.

Results: 1364 references were retrieved using initial searching strategy, and 20 epidemiological publications were selected for data extraction following inclusion/ exclusion criteria. The identified studies reported prevalence ranging from 3.8 per 100,000 in Taiwan to 50 per 100,000 in the United States. The prevalence was 23 per 100,000 (95\% CI: $16-29$ per 100,$000 ; 18$ studies) in a pooled sample of 11,574 individuals. Incidence of systemic sclerosis ranges from 0.77 per 100,000 in the Netherlands to 5.6 per 100,000 in the United States. Systemic sclerosis predominates, with higher prevalence and incidence rates, in females than in males. It's important to note that different methodologies were used to derive these numbers, so comparisons were made with caution.

Conclusion: This review provides an updated literature review on the current estimates of disease prevalence and incidence of systemic sclerosis in different countries. The review reports slightly higher incidence/prevalence compared to previous studies.

(C) 2019 Aurora Health Care, Inc. 